\title{
Stereoselective Synthesis of Chiral Oxepane and Pyran through Intramolecular Nitrone Cycloaddition in Organized Aqueous Media
}

\author{
Amrita Chatterjee and Pranab Kumar Bhattacharya* \\ Chemistry Division, Indian Institute of Chemical Biology, 4, Raja S. C. Mullick Road \\ Jadavpur, Kolkata 700 032, India.
}

Supporting Information

Table of Content

I. General Remarks

S 2

II. General procedure for the preparation of 3-O-allyl furanoside derivatives..... S 2

III. General procedure for the preparation of 3-O-allylfuranoside-5-aldehydes... S 2

IV. A Typical experimental procedure for surfactant catalyzed intramolecular Nitrone cycloaddition reaction in aqueous media.............................. S 2

V. Dynamic Light Scattering Study............................................. S 3

VI. Characterization of the isoxazolidines products .............................. S 4

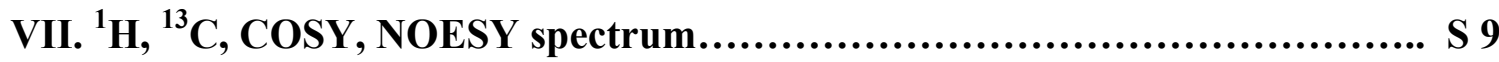


General Remarks: Melting points are uncorrected, the reagents were obtained from commercial sources and used without purification. The solvents were used as technical grade, and freshly distilled prior use. NMR studies were carried out with $500 \mathrm{MHz}$ spectrometers. Tetramethyl silane (TMS) served as internal standard $(\delta=0)$ for ${ }^{1} \mathrm{H}$ NMR and $\mathrm{CDCl}_{3}$ as internal standard $(\delta=77.0)$ for ${ }^{13} \mathrm{C}$ NMR. Chemical shifts are stated in parts per million. COSY and NOESY experiments have been carried out in order to assign the $1 \mathrm{H}$ spectra completely. IR spectra were measured using $\mathrm{KBr}$ pellet, MS spectra were measured in EIMS mode, optical rotations were measured with polarimeter at $25^{\circ} \mathrm{C}$. The surfactants used are Cetyl Trimethyl Ammonium Bromide (CTAB), Sodium Dodecyl Sulfate (SDS), Sodium Dodecyl Benzene Sulfonate (SDBS), Dodecyl Benzene Sulfonic Acid (DBSA), Polyoxyethylene(10) isooctylphenyl ether (Triton X-100), Benzyl-polyethyleneglycol tert-octylphenyl ether (Triton CF 10), Polyoxyethylene(20) sorbitan monolaurate (Tween 20)

General procedure for the preparation of 3- $O$-allyl furanoside derivatives ${ }^{1,2}$ 1(a-f) : To a stirred solution of sodium hydride ( $2.2 \mathrm{mmol}, 50 \%$ suspension) in DMF (10 $\mathrm{mL}), 1,2: 5,6$-diisopropylidene furanose $(1 \mathrm{mmol})$ in DMF $(5 \mathrm{~mL})$ was added slowly at $0^{\circ} \mathrm{C}$ in a $50 \mathrm{~mL}$ two neck round bottom flask. After stirring at room temperature for 30 min, allyl bromide or crotyl bromide or prenyl bromide $(1.5 \mathrm{mmol})$ was added and reaction mixture was stirred for further $3 \mathrm{hr}$. The reaction was quenched with aqueous ammonium chloride solution $(10 \mathrm{~mL})$ and extracted with ether $(3 \times 10 \mathrm{~mL})$. The ether layer was washed with water $(15 \mathrm{~mL})$, brine $(15 \mathrm{~mL})$ and dried over $\mathrm{Na}_{2} \mathrm{SO}_{4}$. The organic layer was evaporated and residue obtained purified by column chromatography over silica gel to afford $\mathbf{1}(\mathbf{a}-\mathbf{f})$.

General procedure for the preparation of 3-O-allylfuranoside-5-aldehydes ${ }^{2}$ 3(a-f): A solution of the 3-O-allyl diisopropylidene derivatives, $\mathbf{1}(\mathbf{a - f}),(1.5 \mathrm{~mL})$ in aqueous $\operatorname{AcOH}(75 \%, 10 \mathrm{~mL})$ was stirred for $15 \mathrm{~h}$ at $25^{\circ} \mathrm{C}$. The reaction mixture was then evaporated and the residue was coevaporated with dry toluene. The oil thus obtained was chromatographed over silica gel using EtOAc as the eluent and the diol (2 a-f) obtained as a syrupy liquid was dried under vacuum and used for the next step without any further purification. To a solution of the above diol $(2 \mathbf{a - f})(1 \mathrm{mmol})$ in $\mathrm{CH}_{3} \mathrm{OH}(10 \mathrm{~mL})$ was added dropwise at $0^{\circ} \mathrm{C}$ a solution of $\mathrm{NaIO}_{4}(1.2 \mathrm{mmol})$ in water $(10 \mathrm{~mL})$. The solution was allowed to warm upto $25^{\circ} \mathrm{C}$ and stirring was continued for $3 \mathrm{hr}$. after filtration of the reaction mixture, the filtrate was concentrated. Extraction with $\mathrm{CHCl}_{3}$ and evaporation of the solvent afforded the aldehyde (3 a-f), which was used immediately without further purification.

\section{A Typical experimental procedure for surfactant catalyzed intramolecular nitrone cycloaddition reaction in aqueous media.}

To the aldehyde (3 a-f) $(0.5$ mmole) were added a solution of CTAB (10 mol \%) in $\mathrm{H}_{2} \mathrm{O}(2 \mathrm{~mL})$ and phenyl hydroxylamine $(0.6 \mathrm{mmole}, 1.2$ equiv) successively at room temperature in a $25 \mathrm{~mL}$ round bottom flask. The reaction mixture was sonicated for $5 \mathrm{~min}$ 
and allowed to stir at room temperature. The reaction was monitored by TLC. After stirring for the period of time listed in Table, the product was extracted with ethyl acetate, washed with brine, dried over $\mathrm{Na}_{2} \mathrm{SO}_{4}$, and concentrated. Purification of the residue by silica gel chromatography gave the desired product.

\section{Dynamic Light Scattering Study of Cetyl Trimethyl Ammonium Bromide:}

The experiment was done with the freshly filtered solution of $0.05 \mathrm{mmol}$ of $\mathrm{CTAB}$ in double distilled water. The solution was sonicated in a bath sonicator for 5 mins and kept at room temperature for 2 hours, for hydration, as a standard practice. The study shows that surfactant molecule in aqueous solution, self assembled with hydrodynamic radii $1.296 \mathrm{~nm}(29.36 \%)$ and $295.3 \mathrm{~nm}(70.64 \%)$

Size Distribution by Intensity

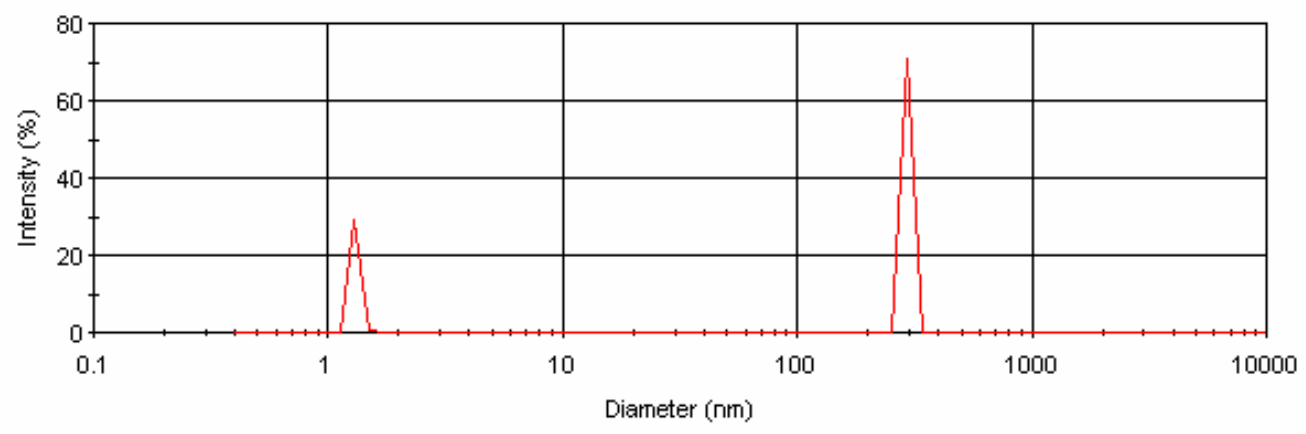


Characterization of the isoxazolidines products

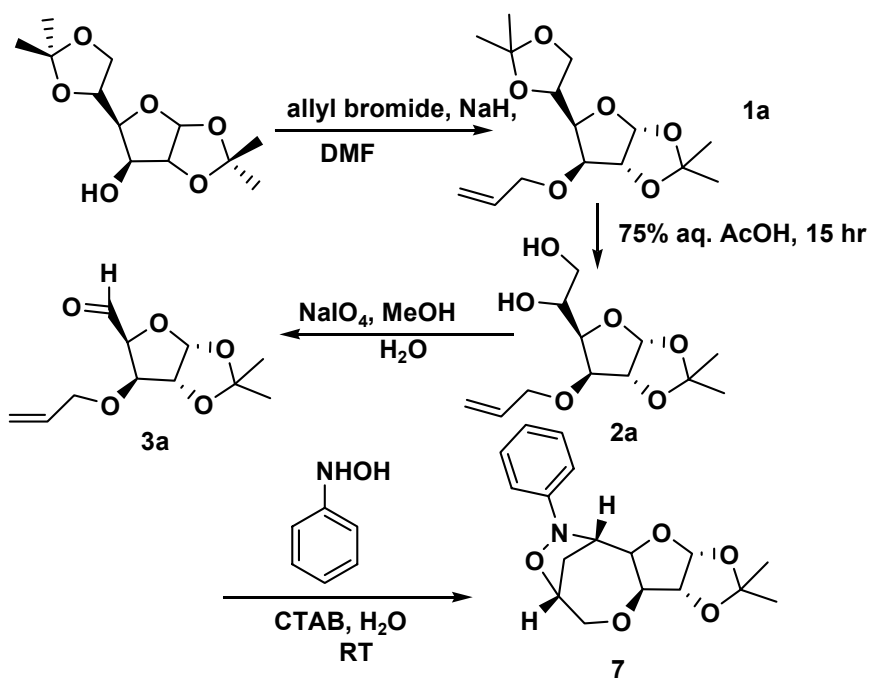

Spectral data of 7: (2aS,2bR,4R,5R,5aS,7aR)-2-Phenyl-2b,4,5,5a-tetrahydro-2-aza1,6-dioxa-4,5-isopropylidenedioxyfuro-[2',3':4,5]bicyclo[4.2.1]nonane : ${ }^{1} \mathrm{H}$ NMR $\left(\mathrm{CDCl}_{3}, 500 \mathrm{MHz}\right): 1.33(3 \mathrm{H}, \mathrm{s}), 1.54(3 \mathrm{H}, \mathrm{s}), 1.90-1.96(1 \mathrm{H}, \mathrm{m}), 2.55(1 \mathrm{H}, \mathrm{d}, J=12.16$ $\mathrm{Hz}), 3.71(1 \mathrm{H}, \mathrm{dd}, J=2.58,12.97 \mathrm{~Hz}), 3.75(1 \mathrm{H}, \mathrm{d}, J=12.9 \mathrm{~Hz}), 4.18(1 \mathrm{H}, \mathrm{d}, J=1.02$ $\mathrm{Hz}), 4.29(1 \mathrm{H}, \mathrm{dd}, J=3.3,5.97 \mathrm{~Hz}), 4.43(1 \mathrm{H}, \mathrm{bs}), 4.48(1 \mathrm{H}, \mathrm{d}, J=3.69 \mathrm{~Hz}), 4.70(1 \mathrm{H}$, $\mathrm{dd}, J=2.19,8.96 \mathrm{~Hz}), 5.92(1 \mathrm{H}, \mathrm{d}, J=3.5 \mathrm{~Hz}), 7.00(1 \mathrm{H}, \mathrm{t}, J=7.5 \mathrm{~Hz}), 7.06(2 \mathrm{H}, \mathrm{d}, J=$ 7.5 Hz), 7.26-7.29 (2H, m); ${ }^{13} \mathrm{C} \mathrm{NMR}\left(\mathrm{CDCl}_{3}, 75 \mathrm{MHz}\right): 26.0,26.6,26.9,66.2,71.8$, 78.3, 79.4, 82.8, 84.5, 104.2, 111.7, 115.2, 122.8, 128.9, 151.2; IR (KBr): 3063, 2987, 2939, 2898, 1594, 1489, 1450, 1376, 1294, 1226, 1146, 1092, 1010, 956, 858, 776, 704 $\mathrm{cm}^{-1}$; EIMS: $319\left(\mathrm{M}^{+}\right), 304,232,183,160 ;[\alpha]_{\mathrm{D}}^{25}-183.49\left(\mathrm{c}=1.43, \mathrm{CHCl}_{3}\right) ; \mathrm{mp}: 89^{\circ} \mathrm{C}$

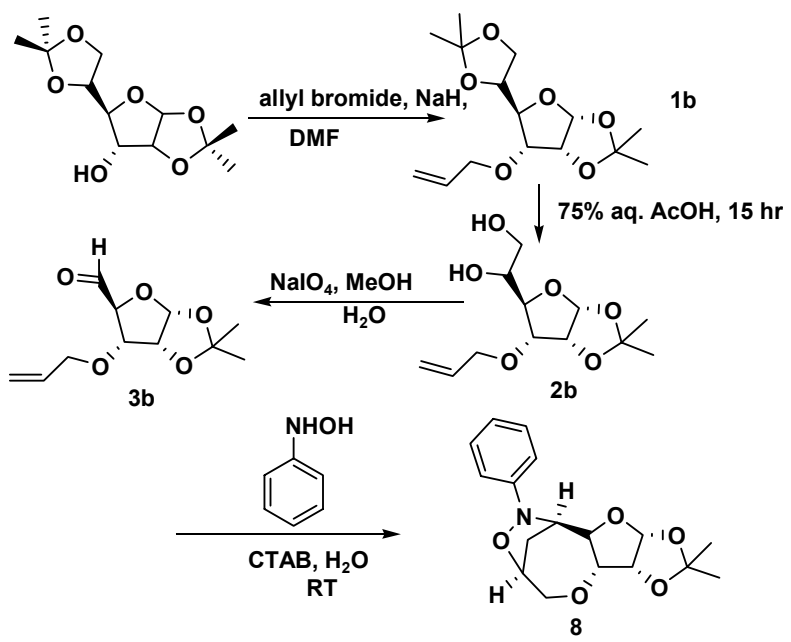

Spectral data of 8: (2aR,2bR,4R,5R,5aR,7aS)-2-Phenyl-2b,4,5,5a-tetrahydro-2-aza1,6-dioxa-4,5-isopropylidenedioxyfuro-[2',3':4,5]bicyclo[4.2.1]nonane : ${ }^{1} \mathrm{H} N \mathrm{NM}$ $\left(\mathrm{CDCl}_{3}, 500 \mathrm{MHz}\right): 1.37(3 \mathrm{H}, \mathrm{s}), 1.64(3 \mathrm{H}, \mathrm{s}), 2.16(1 \mathrm{H}, \mathrm{d}, J=13.0 \mathrm{~Hz}), 2.29(1 \mathrm{H}, \mathrm{ddd}$, $J=7.5,9.0,12.5 \mathrm{~Hz}), 3.82(1 \mathrm{H}, \mathrm{dd}, J=2.6,13.0 \mathrm{~Hz}), 3.98(1 \mathrm{H}, \mathrm{dd}, J=2.5,9.5 \mathrm{~Hz}), 4.02$ 
$(1 \mathrm{H}, \mathrm{d}, J=13 \mathrm{~Hz}), 4.20(1 \mathrm{H}, \mathrm{dd}, J=4.5,9.5 \mathrm{~Hz}), 4.23(1 \mathrm{H}, \mathrm{dd}, J=2.5,7.5 \mathrm{~Hz}), 4.64(1 \mathrm{H}$, $\mathrm{t}, J=3.5 \mathrm{~Hz}), 4.76(1 \mathrm{H}, \mathrm{dd}, J=2.5,9.5 \mathrm{~Hz}), 5.89(1 \mathrm{H}, \mathrm{d}, J=3.5 \mathrm{~Hz}), 6.98-7.01(1 \mathrm{H}, \mathrm{td}$, $J=1.0,7.5 \mathrm{~Hz}), 7.07(2 \mathrm{H}, \mathrm{dd}, J=1,8.5 \mathrm{~Hz}), 7.25-7.28(2 \mathrm{H}, \mathrm{m}) ;{ }^{13} \mathrm{C} \mathrm{NMR}\left(\mathrm{CDCl}_{3}, 125\right.$ MHz): 26.4, 26.8, 28.9, 66.1, 73.0, 77.4, 77.6, 78.2, 78.6, 105.1, 113.7, 116.1, 123.2, 129.2, 152.4; IR (KBr): 2980, 2909, 2862, 1593, 1486, 1448, 1377, 1252, 1211, 1165, 1104, 1061, 1019, 864, 816, 773, $699 \mathrm{~cm}^{-1}$; EIMS: $319\left(\mathrm{M}^{+}\right), 304,262,232,162$;

$[\alpha]_{\mathrm{D}}^{25}+193.33\left(\mathrm{c} 1.14, \mathrm{CHCl}_{3}\right) ; \mathrm{mp}: 134^{\circ} \mathrm{C}$

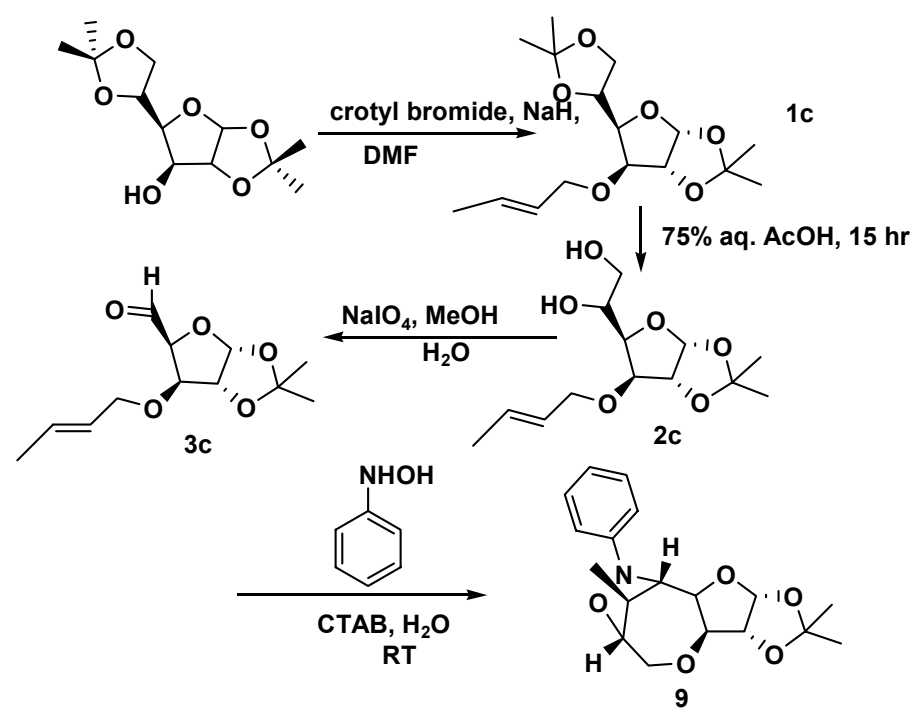

Spectral data of 9: (2aS,2bR, 4R,5R,5aS,7bR,8R)-2-Phenyl-8-methyl-2b,4,5,5atetrahydro-4,5-isopropylidenedioxy-furo[2',3':4,5]-bicyclo[4.2.1] nonane : ${ }^{1} \mathrm{H}$ NMR $\left(\mathrm{CDCl}_{3}, 500 \mathrm{MHz}\right): 0.87(3 \mathrm{H}, \mathrm{d}, J=7.45 \mathrm{~Hz}), 1.32(3 \mathrm{H}, \mathrm{s}), 1.51(3 \mathrm{H}, \mathrm{s}), 3.13(1 \mathrm{H}, \mathrm{q}, J=$ $7.45 \mathrm{~Hz}), 3.69(1 \mathrm{H}, \mathrm{d}, J=13.05 \mathrm{~Hz}), 3.76(1 \mathrm{H}, \mathrm{dd}, J=3.5,13.05 \mathrm{~Hz}), 4.15(1 \mathrm{H}, \mathrm{bs}), 4.18$ $(1 \mathrm{H}, \mathrm{d}, J=3.82 \mathrm{~Hz}), 4.29(1 \mathrm{H}, \mathrm{dd}, J=1.7,3.76 \mathrm{~Hz}), 4.34(1 \mathrm{H}, \mathrm{d}, J=3.36 \mathrm{~Hz}), 4.46(1 \mathrm{H}$, d, $J=3.66 \mathrm{~Hz}), 5.94(1 \mathrm{H}, \mathrm{d}, J=3.66 \mathrm{~Hz}), 6.92(1 \mathrm{H}, \mathrm{t}, J=7.32 \mathrm{~Hz}), 7.06(2 \mathrm{H}, \mathrm{d}, J=7.78$ $\mathrm{Hz})$, 7.24-7.27 (2H, m); ${ }^{13} \mathrm{C} \mathrm{NMR}\left(\mathrm{CDCl}_{3}, 75 \mathrm{MHz}\right): 18.8,26.0,26.6,36.7,71.4,72.0$, 78.7, 82.5, 84.4, 85.2, 104.5, 111.7, 113.5, 121.3, 128.9, 152.1; IR (KBr): 2979, 2934, 1595, 1488, 1455, 1376, 1298, 1247, 1213, 1148, 1086, 1013, 865, 757, $694 \mathrm{~cm}^{-1}$; EIMS: $333(\mathrm{M}+1), 318,299,246,160 ;[\alpha]_{\mathrm{D}}^{25} \quad-110.19\left(\mathrm{c} 1.14, \mathrm{CHCl}_{3}\right) ; \mathrm{mp}: 95^{\circ} \mathrm{C}$ 


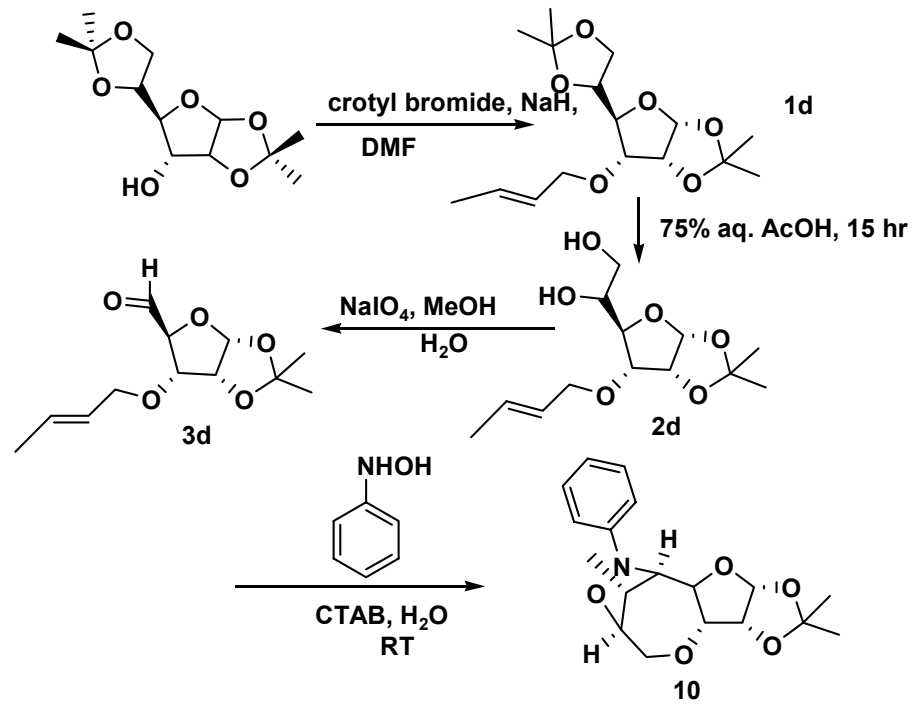

Spectral Data of 10: (2aS,2bR, 4R,5R,5aS,7bS,8S)-2-Phenyl-8-methyl-2b,4,5,5atetrahydro-4,5-isopropylidenedioxy-furo [2',3':4,5]-bicyclo[4.2.1]nonane : ${ }^{1} \mathrm{H} N \mathrm{NM}$ $\left(\mathrm{CDCl}_{3}, 500 \mathrm{MHz}\right): 0.986(3 \mathrm{H}, \mathrm{d}, J=7.3 \mathrm{~Hz}), 1.36(3 \mathrm{H}, \mathrm{s}), 1.64(3 \mathrm{H}, \mathrm{s}), 2.724(1 \mathrm{H}, \mathrm{q}, J=$ $7.3 \mathrm{~Hz}), 3.87(1 \mathrm{H}, \mathrm{dd}, J=3.25,13.1 \mathrm{~Hz}), 3.98(1 \mathrm{H}, \mathrm{d}, J=13.1 \mathrm{~Hz}), 4.03(1 \mathrm{H}, \mathrm{dd}, J=2.0$, $9.3 \mathrm{~Hz}), 4.07$ (1H, bs), $4.1(1 \mathrm{H}, \mathrm{dd}, J=4.26,9.3 \mathrm{~Hz}), 4.34(1 \mathrm{H}, \mathrm{d}, J=3.2 \mathrm{~Hz}), 4.62(1 \mathrm{H}, \mathrm{t}$, $3.79 \mathrm{~Hz}), 5.84(1 \mathrm{H}, \mathrm{d}, J=3.48 \mathrm{~Hz}), 6.9(1 \mathrm{H}, \mathrm{t}, J=7.3 \mathrm{~Hz}), 7.08(2 \mathrm{H}, \mathrm{dd}, J=0.8,8.58$ $\mathrm{Hz}), 7.22-7.26(2 \mathrm{H}, \mathrm{m}) ;{ }^{13} \mathrm{C} \mathrm{NMR}\left(\mathrm{CDCl}_{3}, 75 \mathrm{MHz}\right): 19.2,25.9,26.3,39.2,71.1,73.1$, 76.6, 77.1, 77.7, 85.0, 104.6, 113.2, 113.5, 121.1, 128.7, 152.6; IR (KBr): 2981, 2935, 2858, 1594, 1487, 1454, 1378, 1294, 1248, 1214, 1166, 1118, 1026, 864, $751 \mathrm{~cm}^{-1}$; EIMS: $333\left(\mathrm{M}^{+}\right), 318,276,246,260,176,160 ;[\alpha]_{\mathrm{D}}^{25}+194.43\left(\mathrm{c} 1.15, \mathrm{CHCl}_{3}\right)$; mp: $145^{\circ} \mathrm{C}$ 


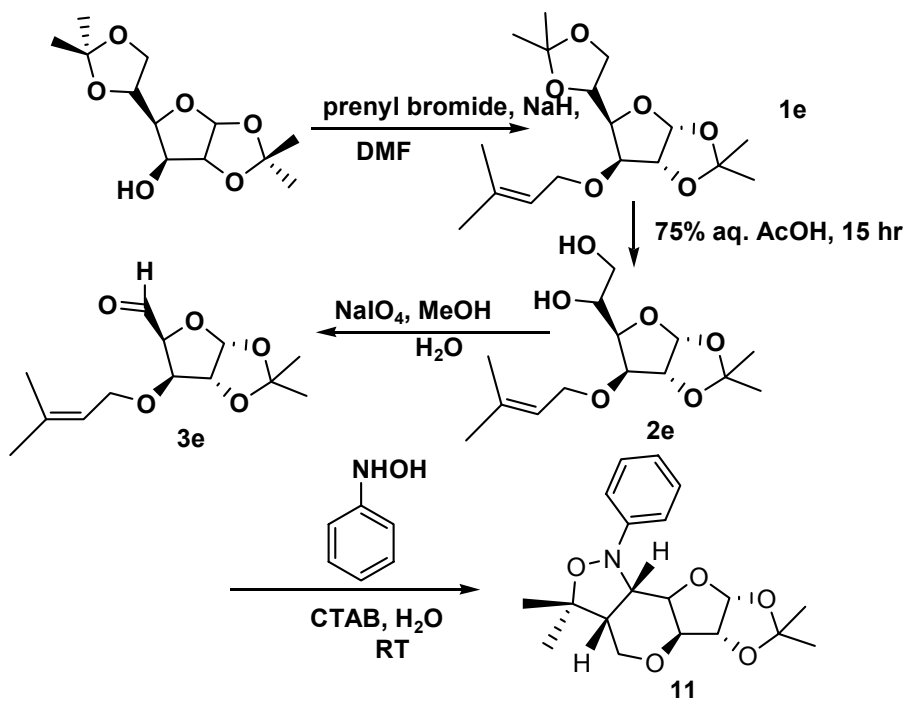

\section{Spectral data of 11: (3aR,5aS,6R,7R,8aR,8bS)-1-Phenyl-3,3-dimethyl-}

1,3,3a,5a,6,7,8a,8b-octahydro-4H-furo[2',3':3,2]pyrano-[4,5-c]isoxazole: ${ }^{1} \mathrm{H}$ NMR $\left(\mathrm{CDCl}_{3}, 500 \mathrm{MHz}\right): 1.04(3 \mathrm{H}, \mathrm{s}), 1.27(3 \mathrm{H}, \mathrm{s}), 1.33(3 \mathrm{H}, \mathrm{s}), 1.49(3 \mathrm{H}, \mathrm{s}), 2.52-2.56(1 \mathrm{H}$, m), $3.56(1 \mathrm{H}, \mathrm{t}, J=11.7 \mathrm{~Hz}), 3.98(1 \mathrm{H}, \mathrm{dd}, J=6.04,11.45 \mathrm{~Hz}), 4.03(1 \mathrm{H}, \mathrm{d}, J=1.33 \mathrm{~Hz})$, $4.12(1 \mathrm{H}, \mathrm{d}, J=5.9 \mathrm{~Hz}), 4.34(1 \mathrm{H}, \mathrm{s}), 4.60(1 \mathrm{H}, \mathrm{d}, J=3.6 \mathrm{~Hz}), 5.92(1 \mathrm{H}, \mathrm{d}, J=3.6 \mathrm{~Hz})$, $6.96(1 \mathrm{H}, \mathrm{t}, J=7.45 \mathrm{~Hz}), 7.05(2 \mathrm{H}, \mathrm{d}, J=7.98 \mathrm{~Hz}), 7.29(2 \mathrm{H}, \mathrm{t}, J=8.2 \mathrm{~Hz})$;

${ }^{13} \mathrm{C} \mathrm{NMR}\left(\mathrm{CDCl}_{3}, 75 \mathrm{MHz}\right): 20.5,26.6,26.8,27.2,45.3,63.1,65.2,75.0,77.7,83.0$, 84.4, 104.7, 112.3, 114.1, 121.8, 153.3; IR (KBr): 2981, 2931, 1596, 1490, 1375, 1215, 1160, 1092, 1021, 838, $755 \mathrm{~cm}^{-1}$; EIMS: $347\left(\mathrm{M}^{+}\right)$, 332, 290, 260, 247, 225, 206, 188, 175,$160 ;[\alpha]_{\mathrm{D}}^{25}-140.84$ (c 2.12, $\mathrm{CHCl}_{3}$ ); mp: $85^{\circ} \mathrm{C}$

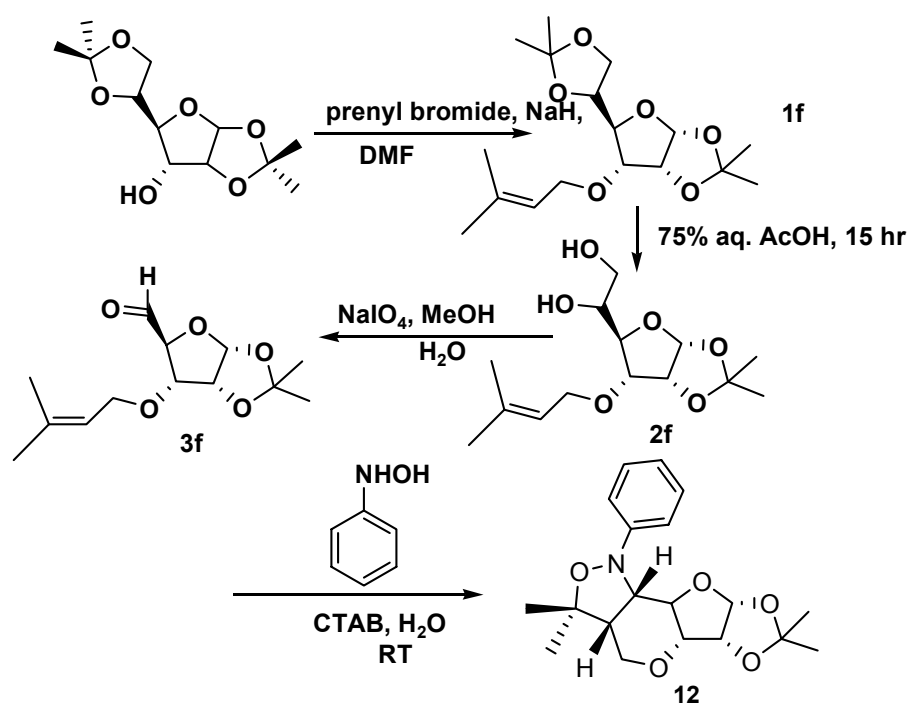


Spectral data of 12: (3aR,5aS,6R,7S,8aR,8bS)-1-Phenyl-3,3-dimethyl-

1,3,3a,5a,6,7,8a,8b-octahydro-4H-furo[2',3':3,2]pyrano-[4,5-c]isoxazole: ${ }^{1} \mathrm{H}$ NMR $\left(\mathrm{CDCl}_{3}, 500 \mathrm{MHz}: 1.33(3 \mathrm{H}, \mathrm{s}), 1.35(3 \mathrm{H}, \mathrm{s}), 1.43(3 \mathrm{H}, \mathrm{s}), 1.58(3 \mathrm{H}, \mathrm{s}), 2.50-2.54(1 \mathrm{H}, \mathrm{m})\right.$, $3.196(1 \mathrm{H}, \mathrm{dd}, J=4.14,10.23 \mathrm{~Hz}), 3.92(1 \mathrm{H}, \mathrm{dd}, J=4.98,12.4 \mathrm{~Hz}), 4.11(1 \mathrm{H}, \mathrm{t}, J=8.7$ $\mathrm{Hz}), 4.13(1 \mathrm{H}, \mathrm{dd}, J=4.13,12.4 \mathrm{~Hz}), 4.31(1 \mathrm{H}, \mathrm{t}, J=9.6 \mathrm{~Hz}), 4.68(1 \mathrm{H}, \mathrm{t}, J=3.8 \mathrm{~Hz})$, $5.85(1 \mathrm{H}, \mathrm{d}, J=3.43 \mathrm{~Hz}), 6.90(1 \mathrm{H}, \mathrm{t}, J=7.26 \mathrm{~Hz}), 7.14(2 \mathrm{H}, \mathrm{d}, J=7.8 \mathrm{~Hz}), 7.21-7.26$ $(2 \mathrm{H}, \mathrm{m}) ;{ }^{13} \mathrm{C} \mathrm{NMR}\left(\mathrm{CDCl}_{3}, 75 \mathrm{MHz}\right): 23.6,25.9,26.0,29.7,49.2,66.7,68.1,72.7,76.3$, $77.9,82.9,104.3,113.2,114.2,120.9,128.3,150.8$;

IR (KBr): 2978, 2934, 2872, 1596, 1490, 1456, 1377, 1308, 1251, 1088, 1012, 865, 760, $698 \mathrm{~cm}^{-1}$; EIMS: $347(\mathrm{M}+1), 332,289,274,260,245,160 ;[\alpha]_{\mathrm{D}}^{25}-100.30\left(\mathrm{c} 2.0, \mathrm{CHCl}_{3}\right)$; mp: $72^{\circ} \mathrm{C}$

\section{References:}

1. Sharma, G. V. M.; Reddy, I. S.; Reddy, V. G.; Rama Rao, A. V. Tetrahedron: Asymmetry, 1999, 10, 229.

2. Bhattacharjee, A.; Datta, S.; Chattopadhyay, P.; Ghoshal, N.; Kundu, A. P.; Pal, A.; Mukhopadhyay, R.; Chowdhury, S.; Bhattacharjya, A.; Patra, A. Tetrahedron 2003, 59, 4623. 

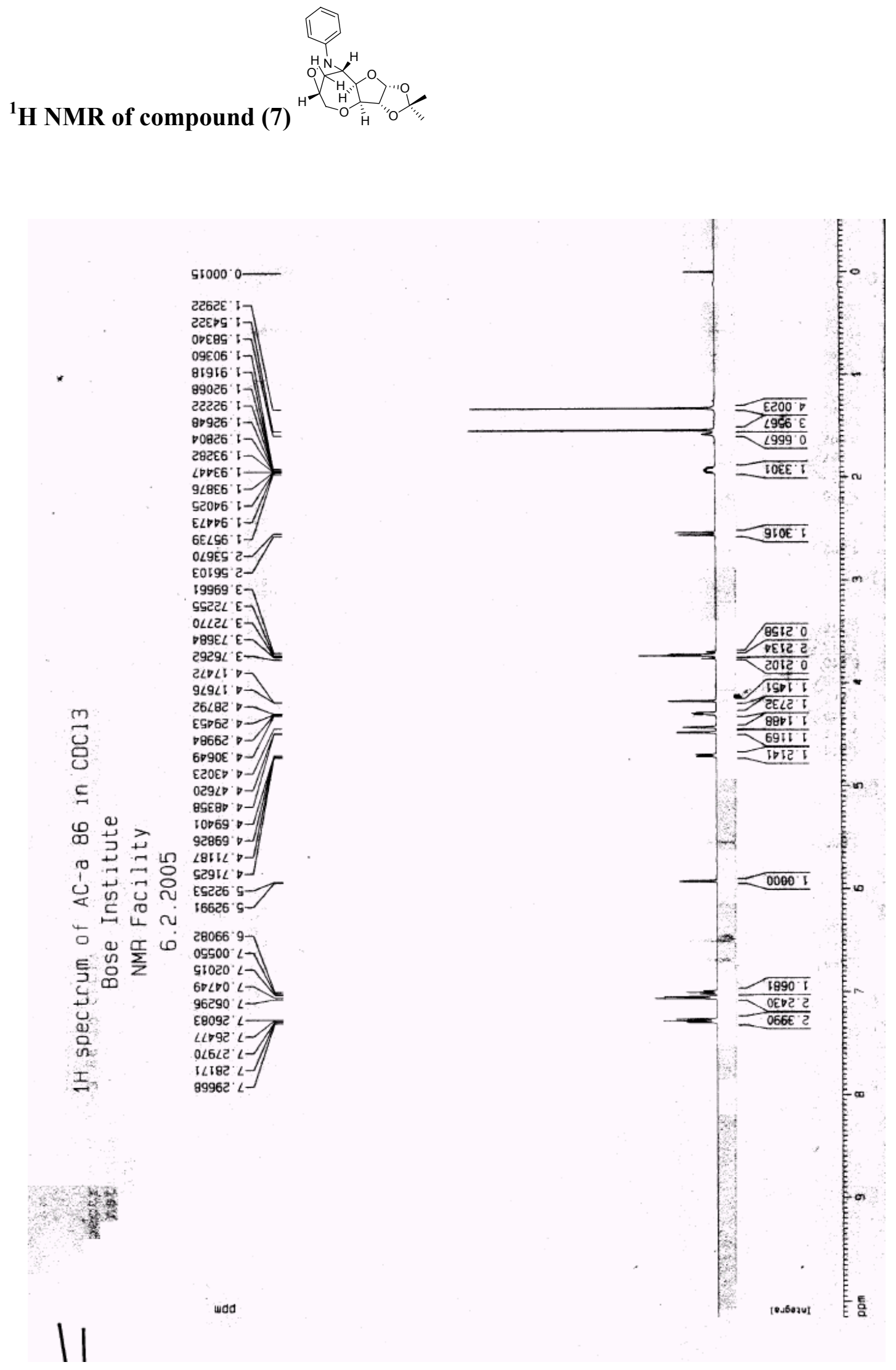
COSY spectrum of (7)

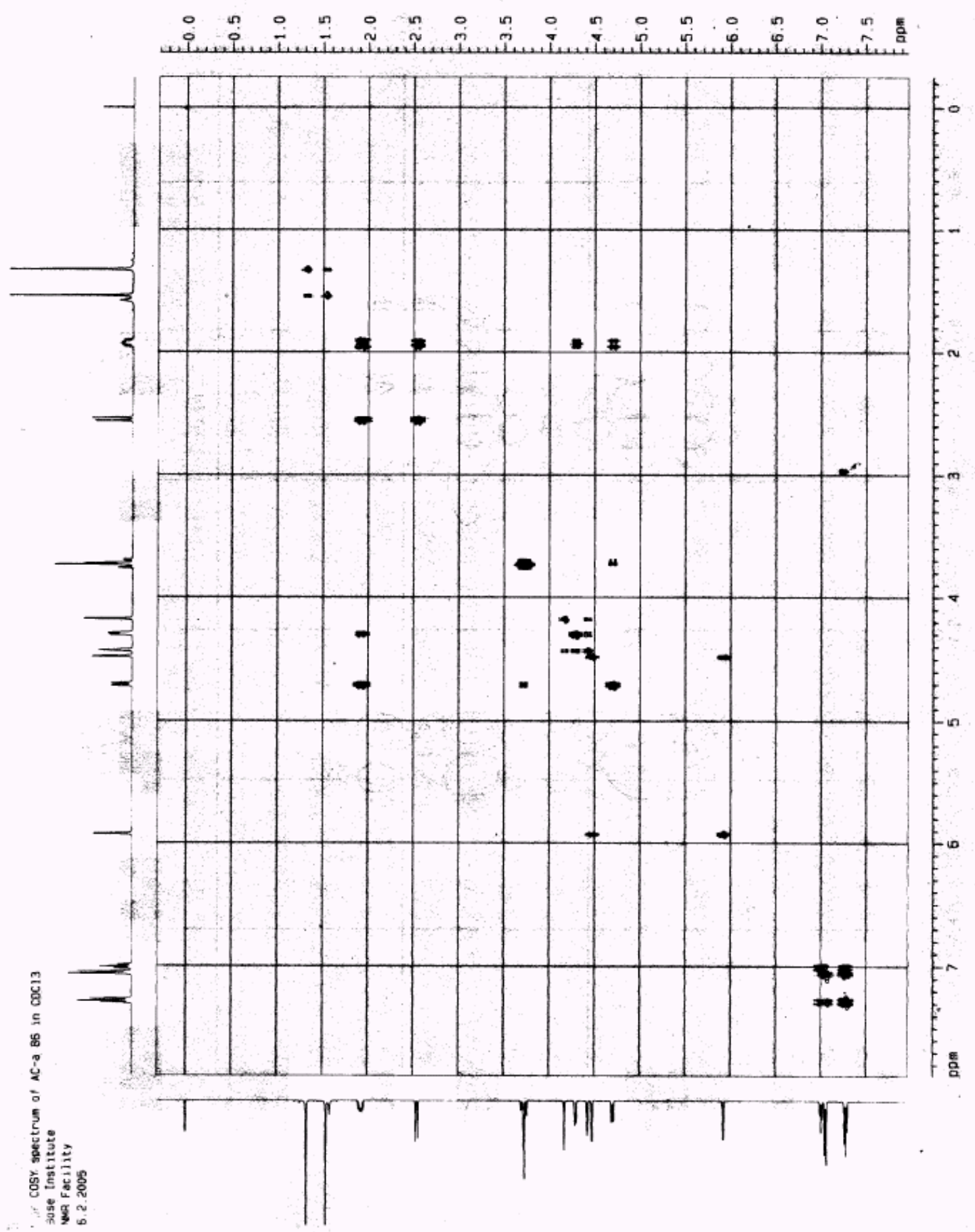


Expansion (1) of COSY spectrum of (7)

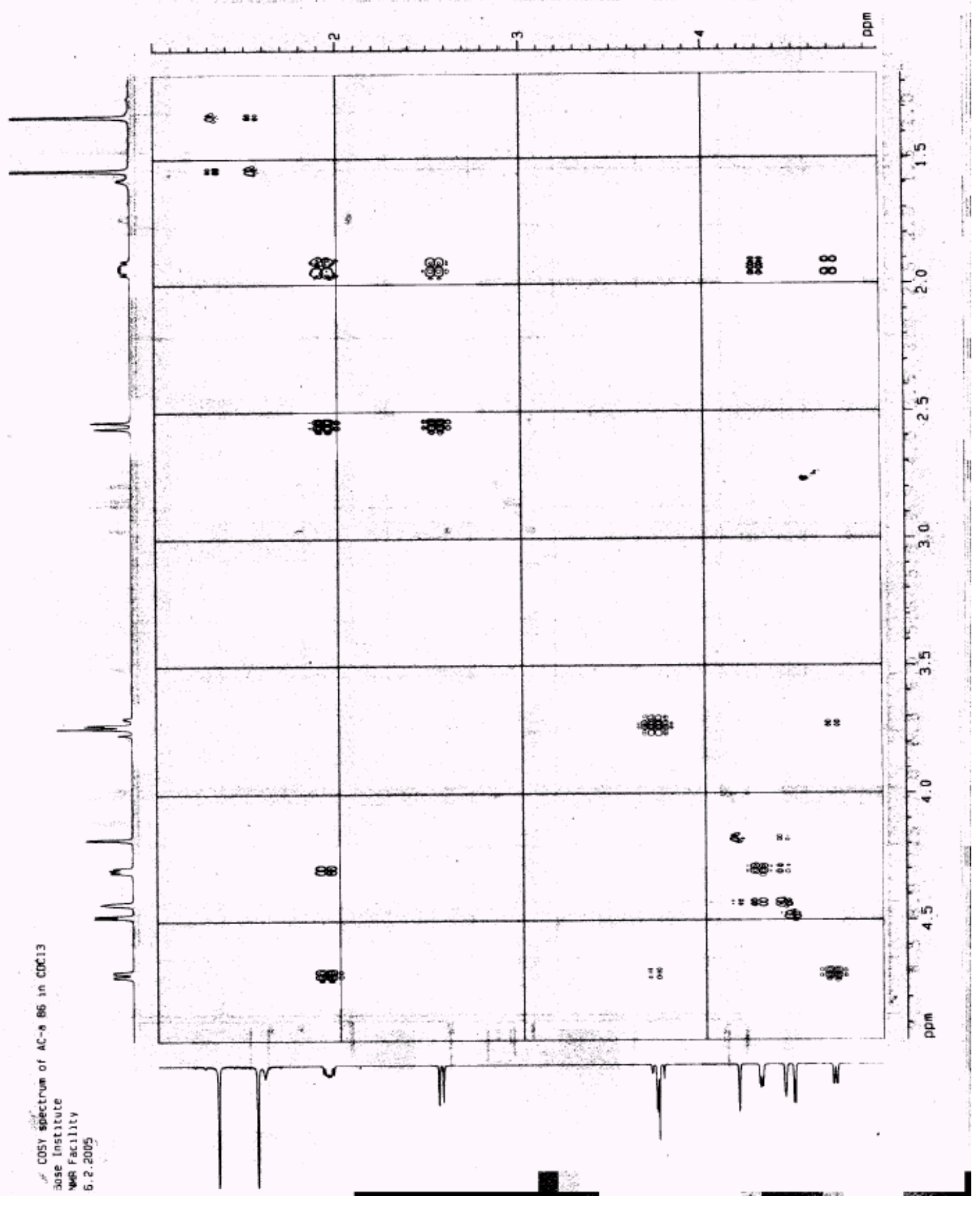


NOESY spectrum of (7)

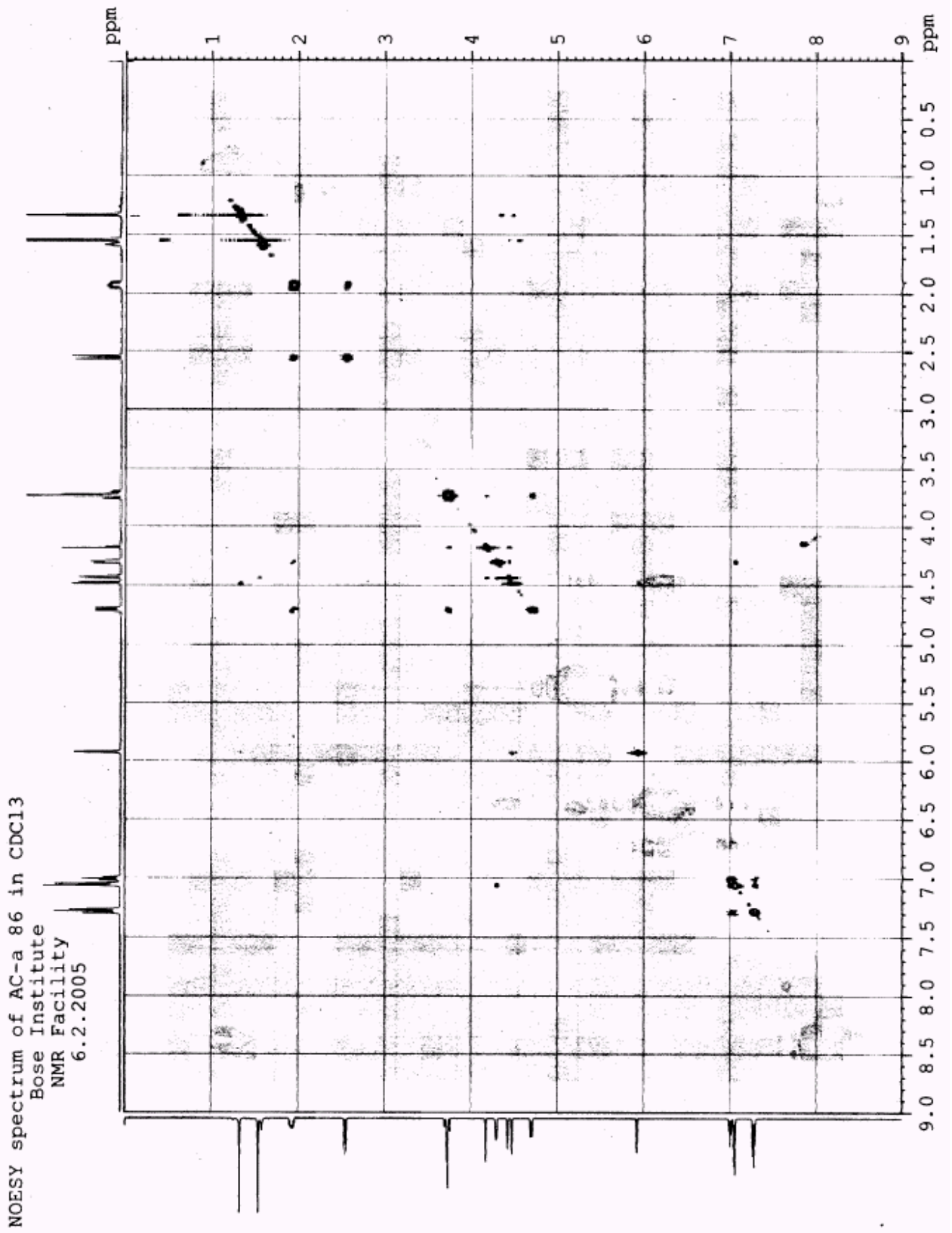


Expansion (1) of NOESY spectrum of (7)

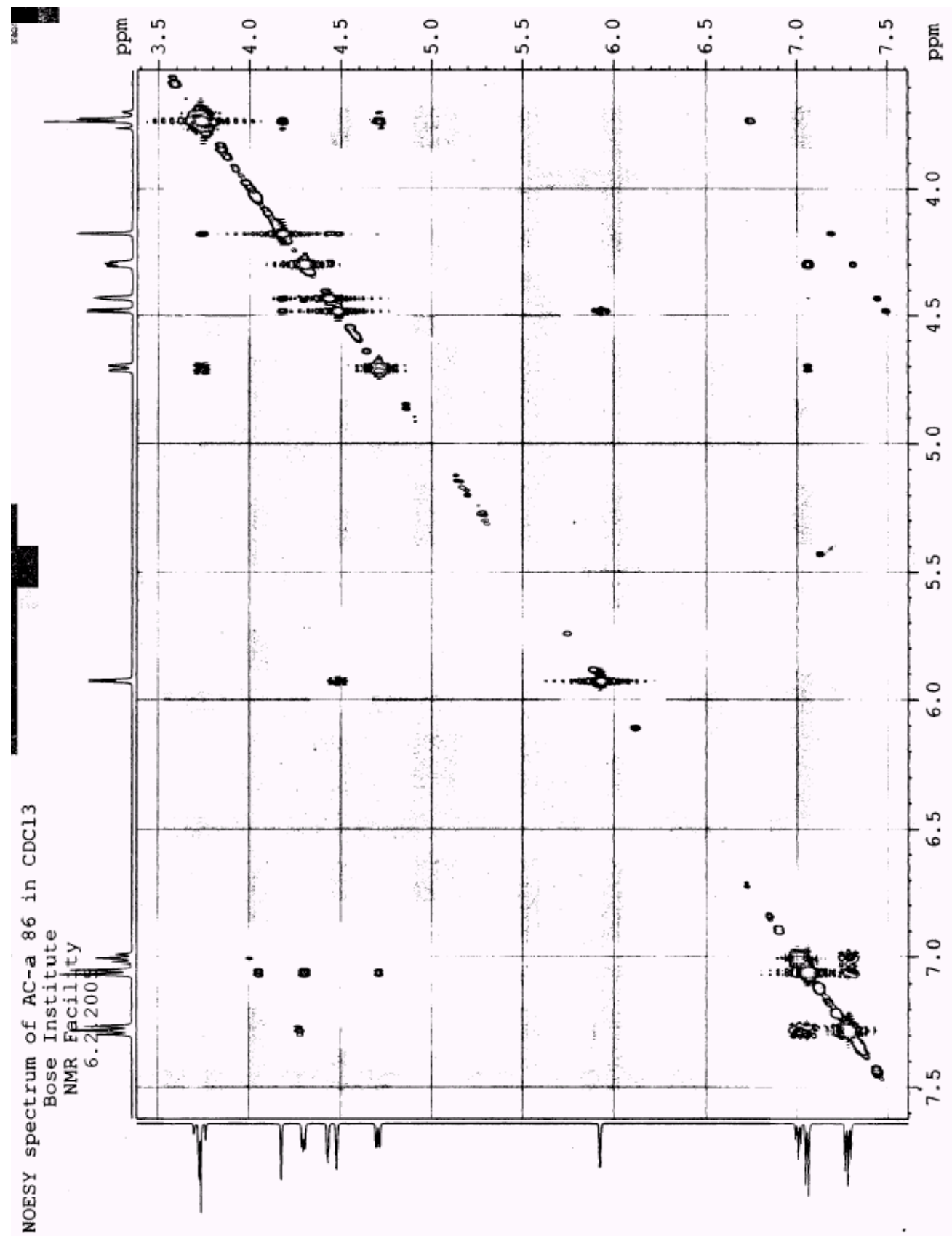




\section{S 14}

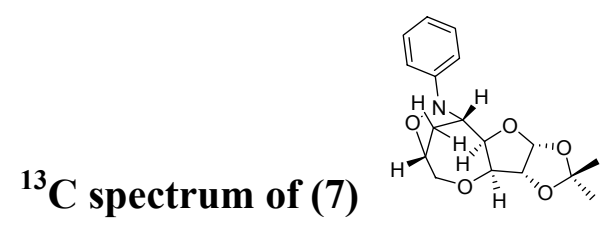

$\varepsilon 00 \cdot 92$
$\varepsilon 95 \cdot 92$
$\angle \varepsilon 6.92$

$865 \cdot 99-$
$\checkmark 9 L \cdot 1 /-$

$685 \cdot 9 L>$

tI0. $L L$

ठ

$\angle E D \angle L$

IVE' $B L$

IGV'6L

टES. จ8

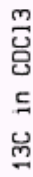

${ }^{2} \angle \mathrm{T} \cdot \mathrm{OOH}-$

$0 \angle 9^{\circ} \mathrm{H}$

gटટ $911-$

$9 \forall L^{\circ} 221-$

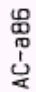

E98. $821-$

96. โร1-

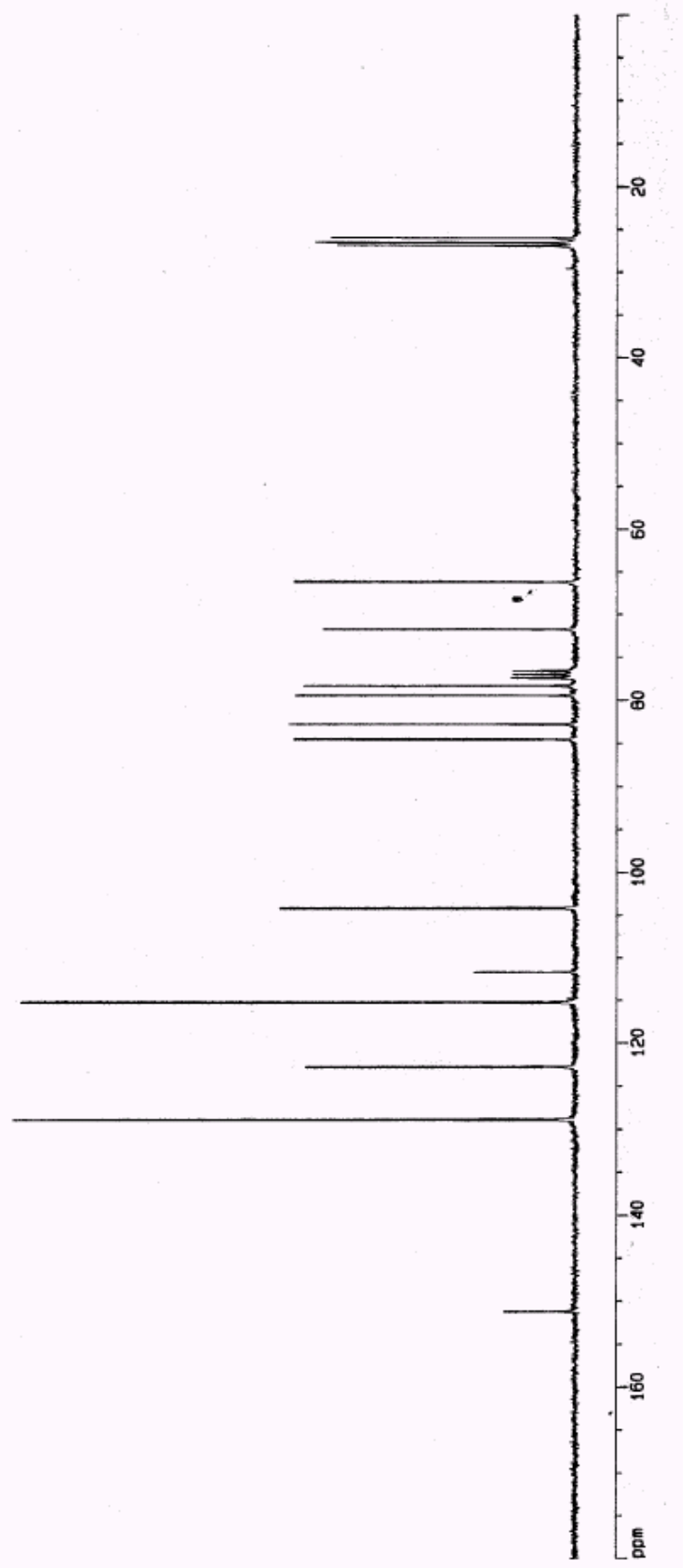



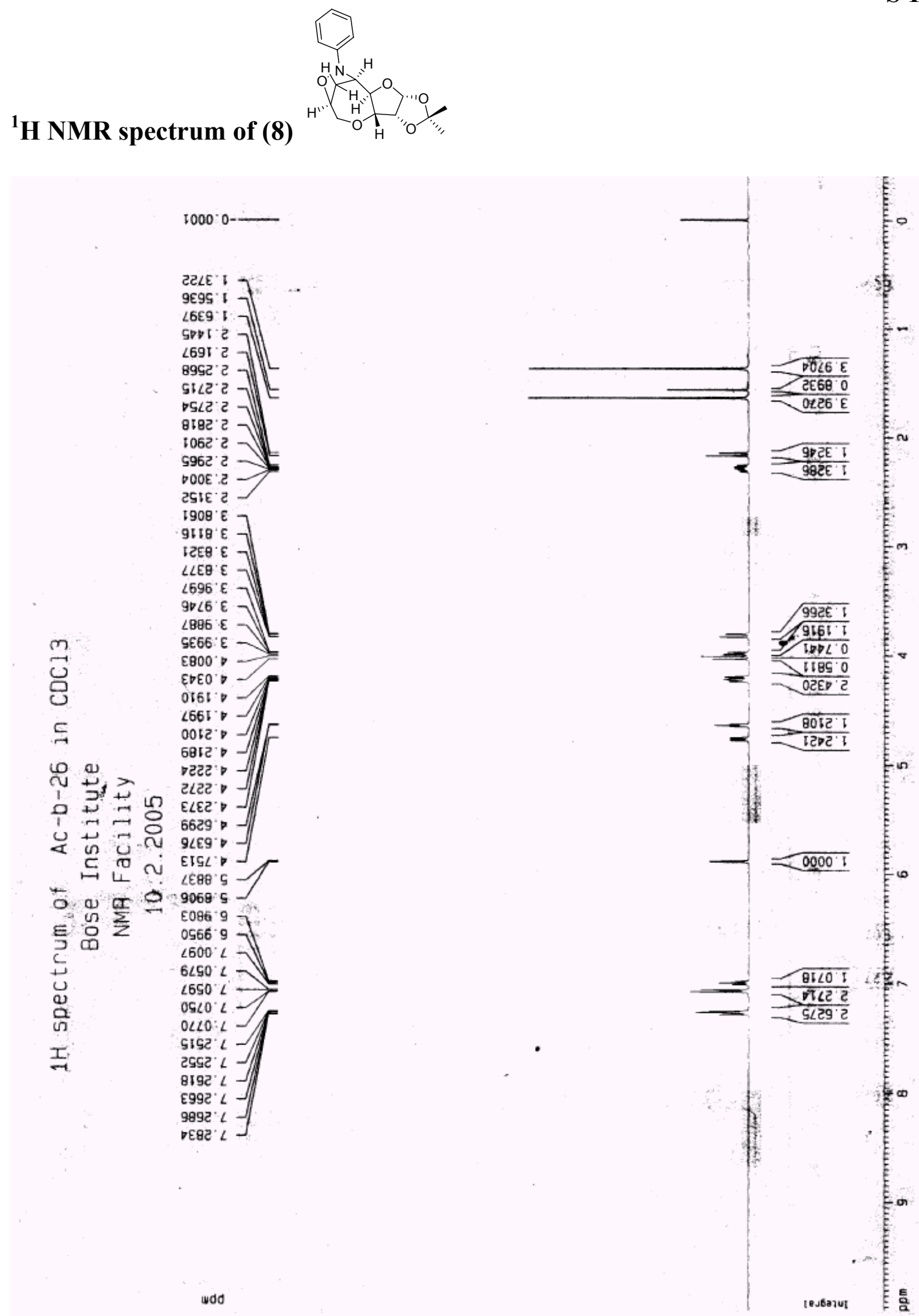

II 

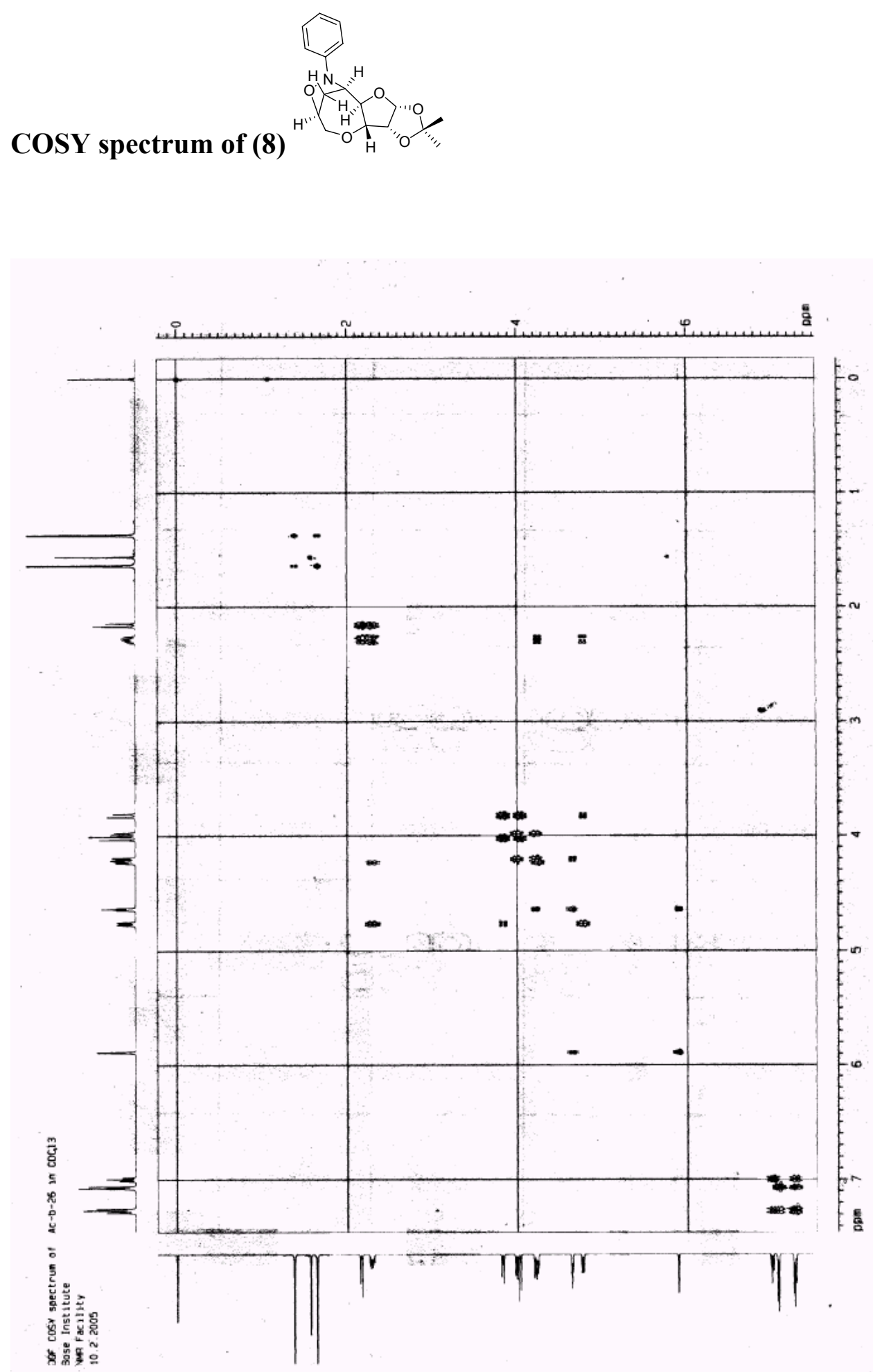

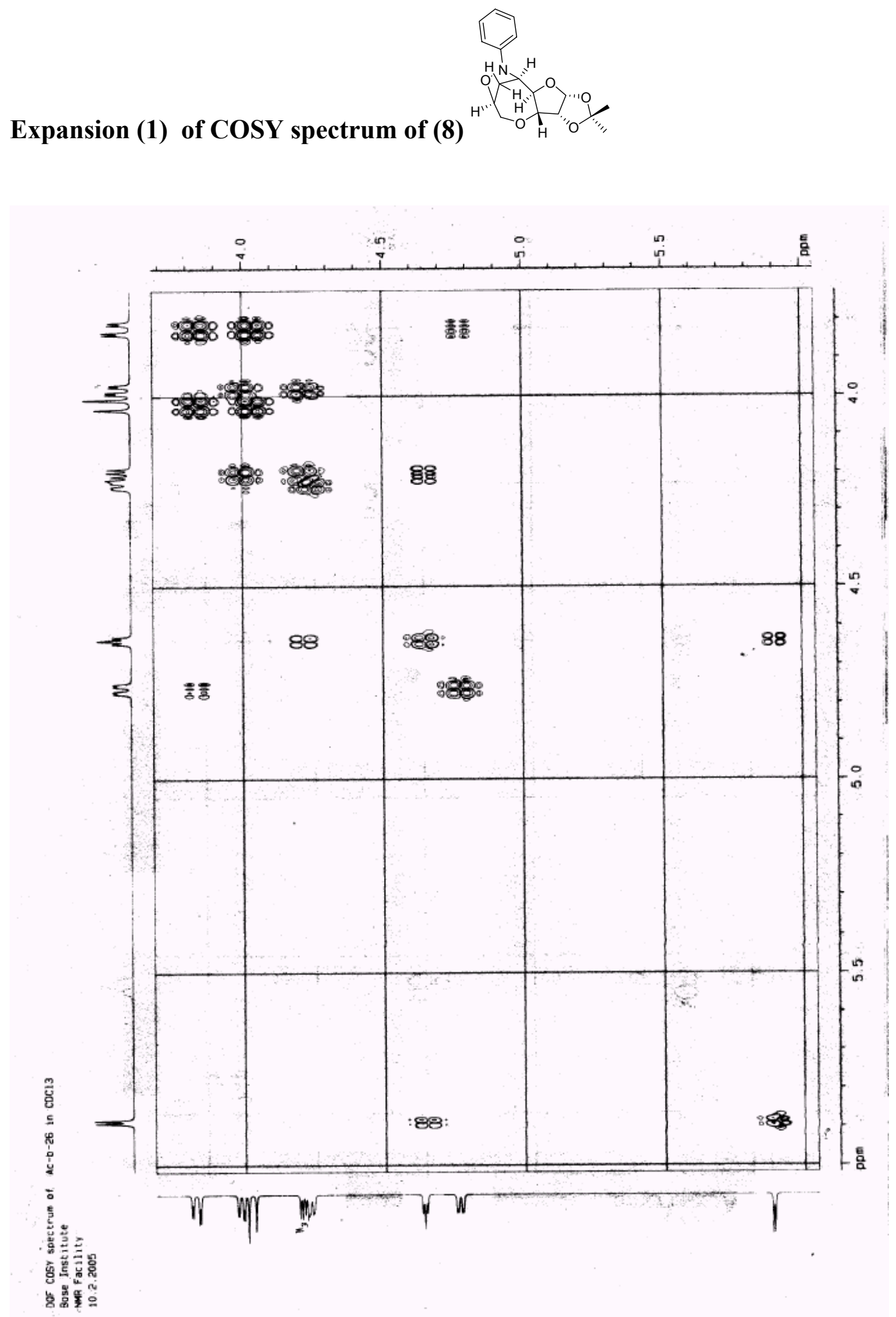
Expansion (2) of COSY spectrum of (8)

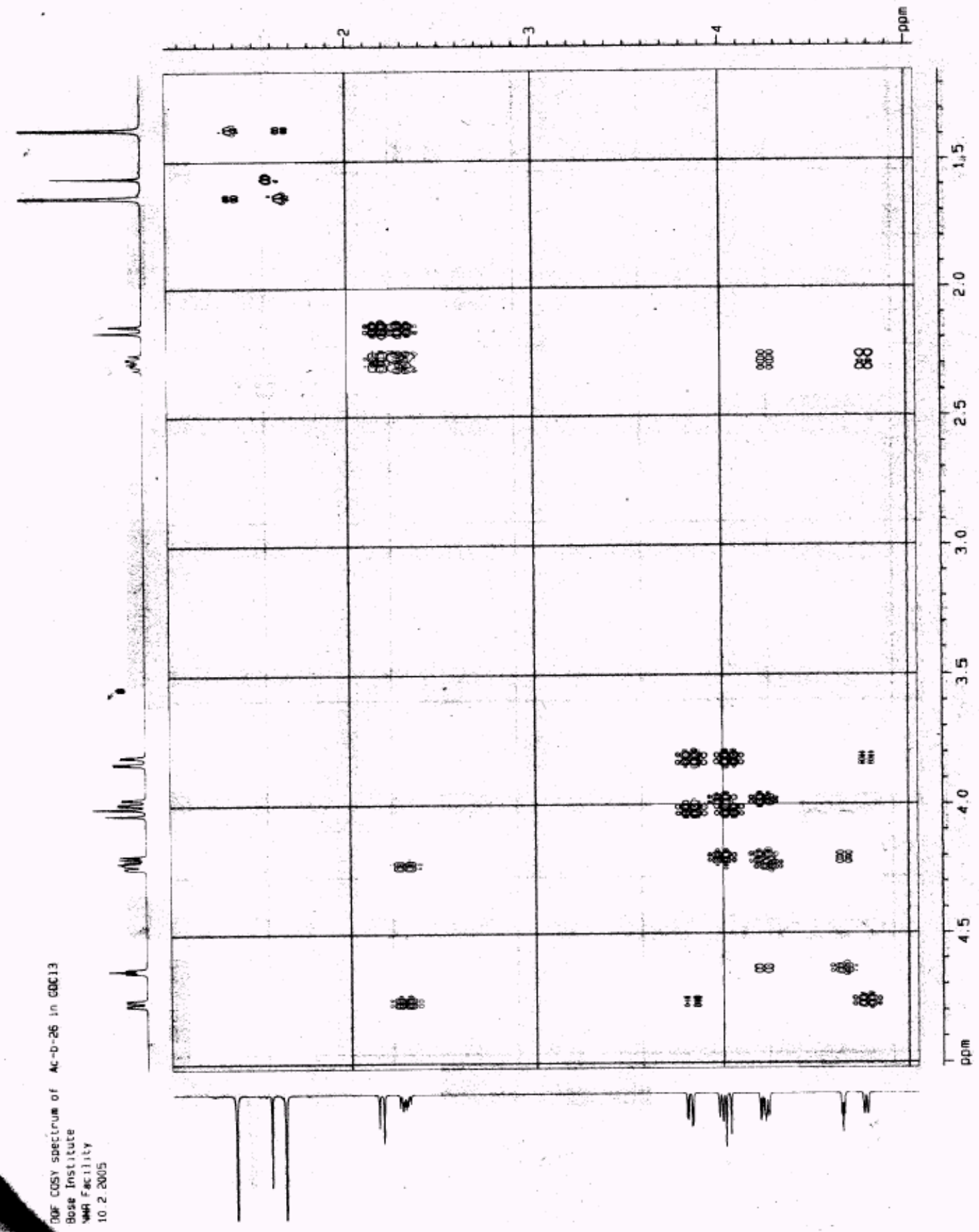



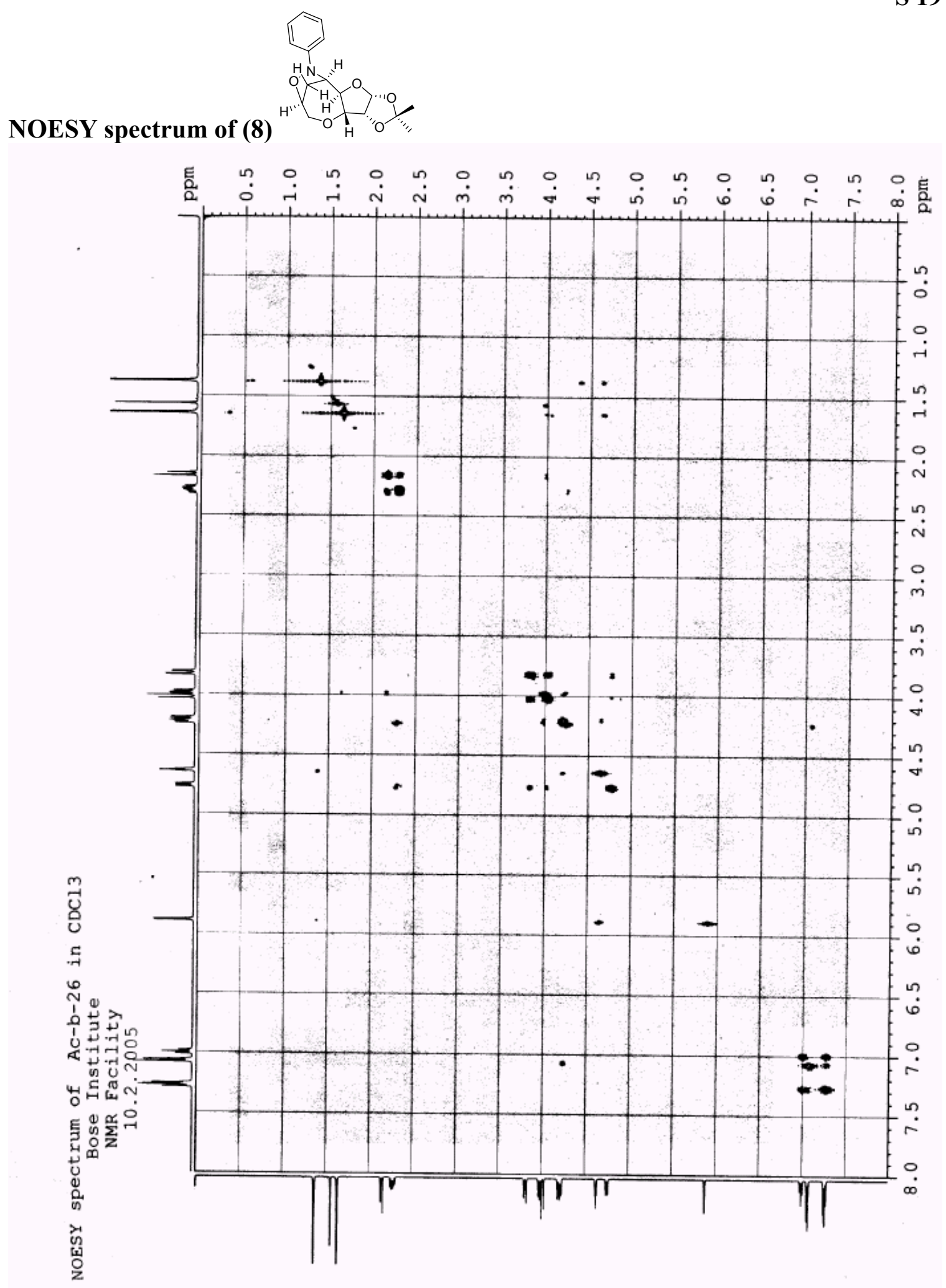
Expansion (1) of NOESY spectrum of (8)

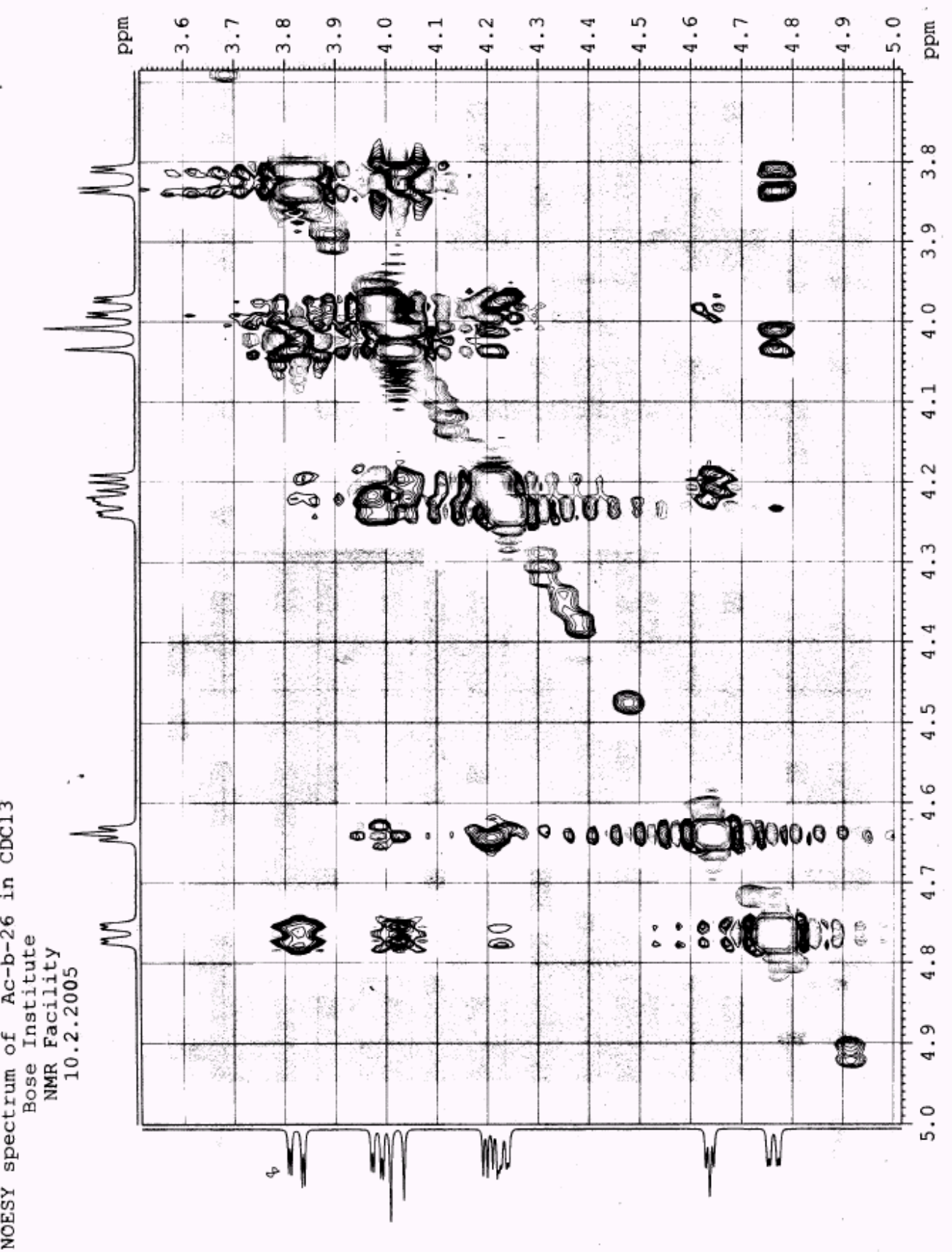


Expansion (2) of NOESY spectrum of (8)

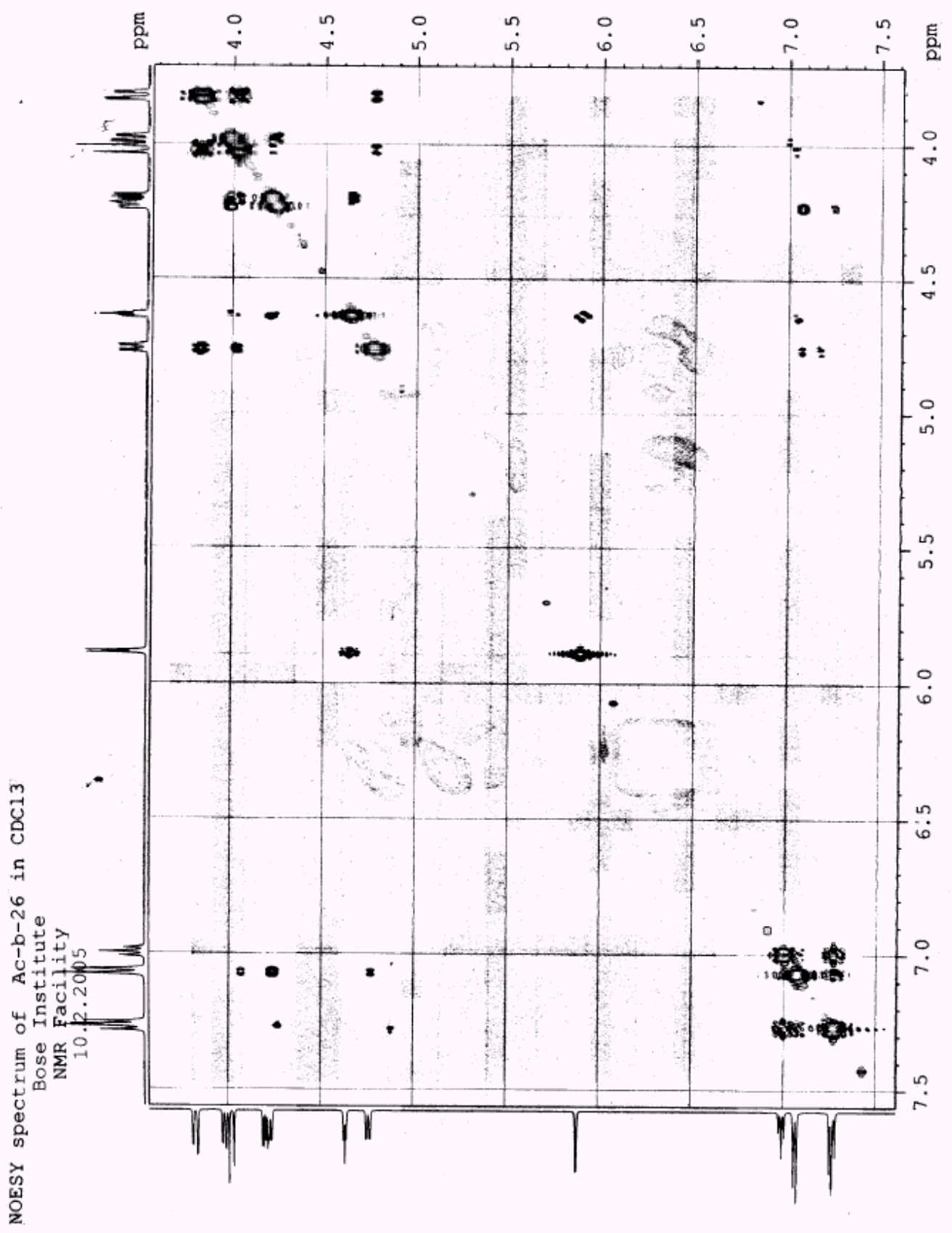




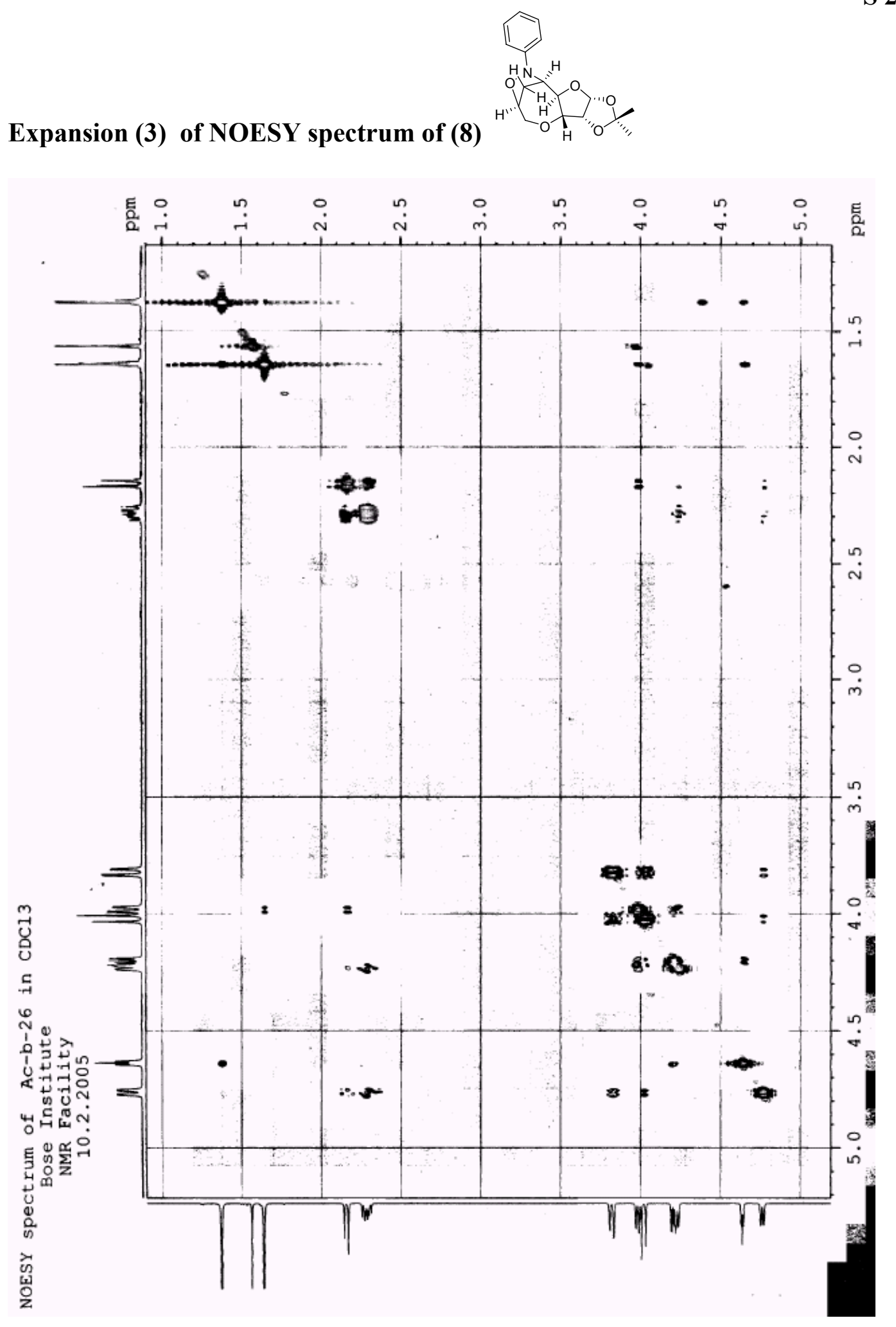




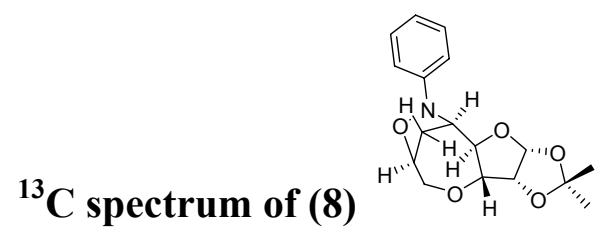

OEB. 95
GEV 95

$\checkmark \varepsilon 6.82=$
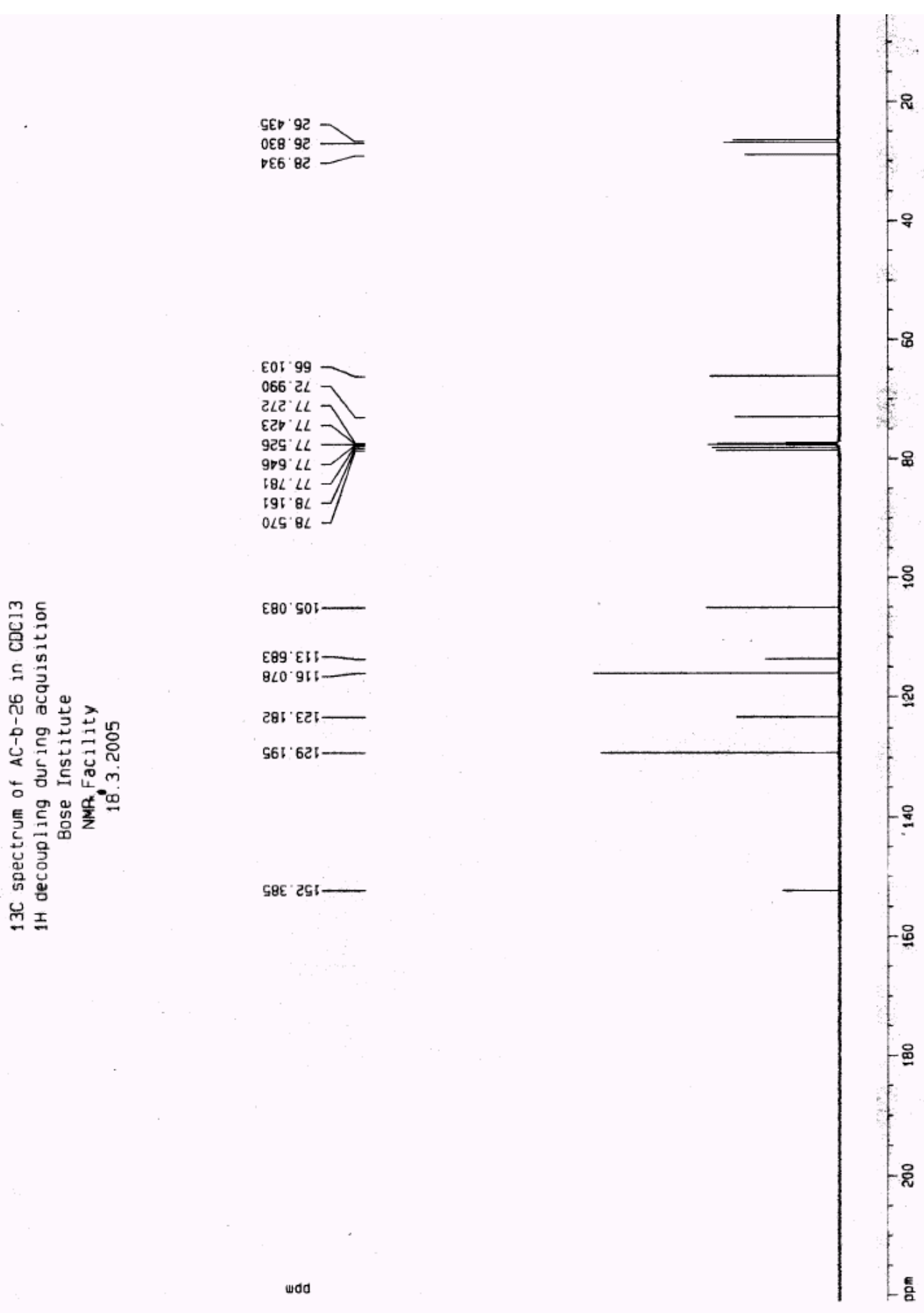

$\varepsilon 80^{\circ} \mathrm{SOL}-$

$\varepsilon 89^{\circ} \mathrm{EL}-$
$8 \angle 0^{\circ} 911-$

टลเ Еटโ-

$561.621-$

५8E' ટ૬ -

吕

足 
${ }^{1}$ H NMR spectrum of (9)
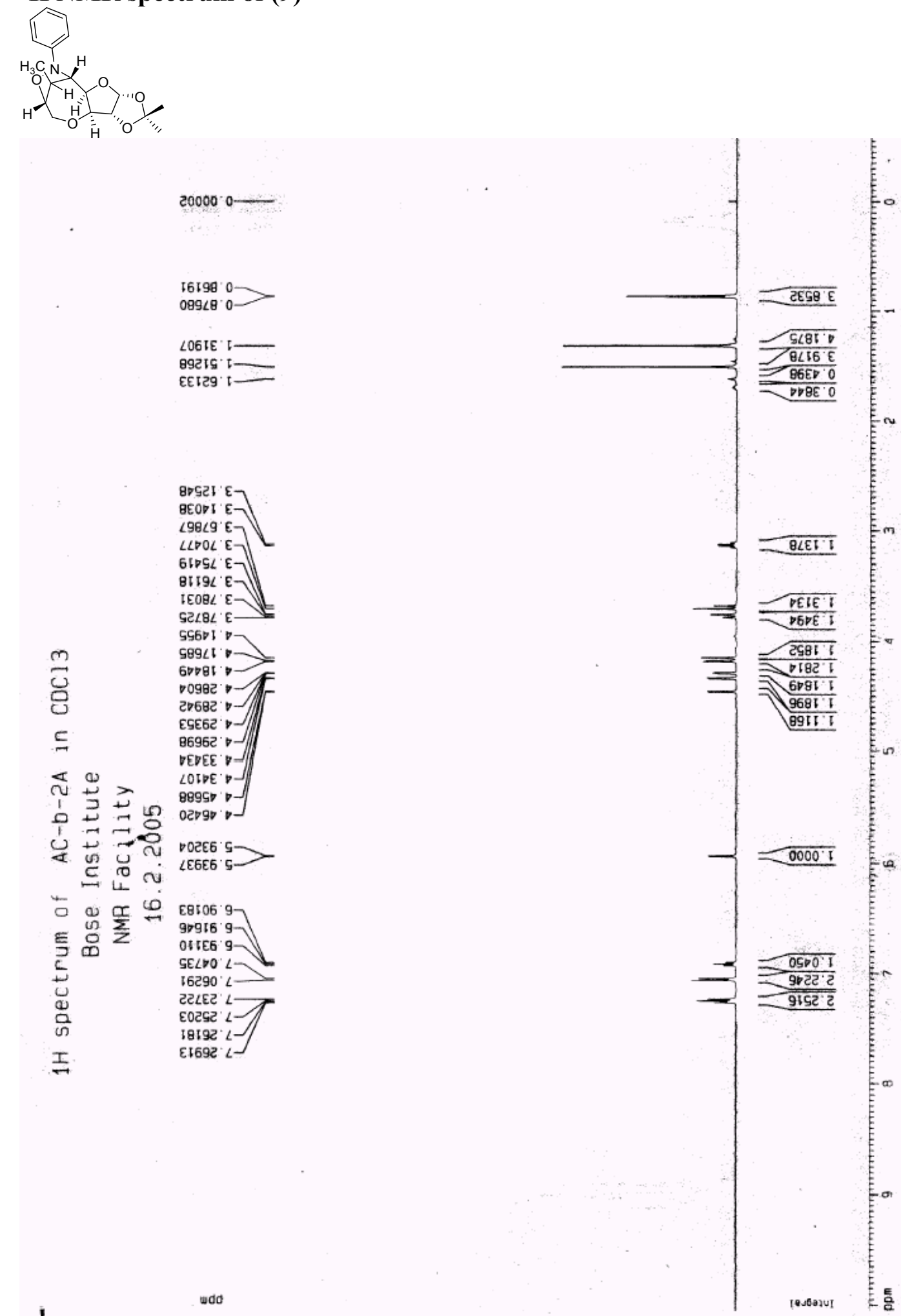
Expansion (1) of ${ }^{1} \mathrm{H}$ NMR spectrum of (9)

S 25
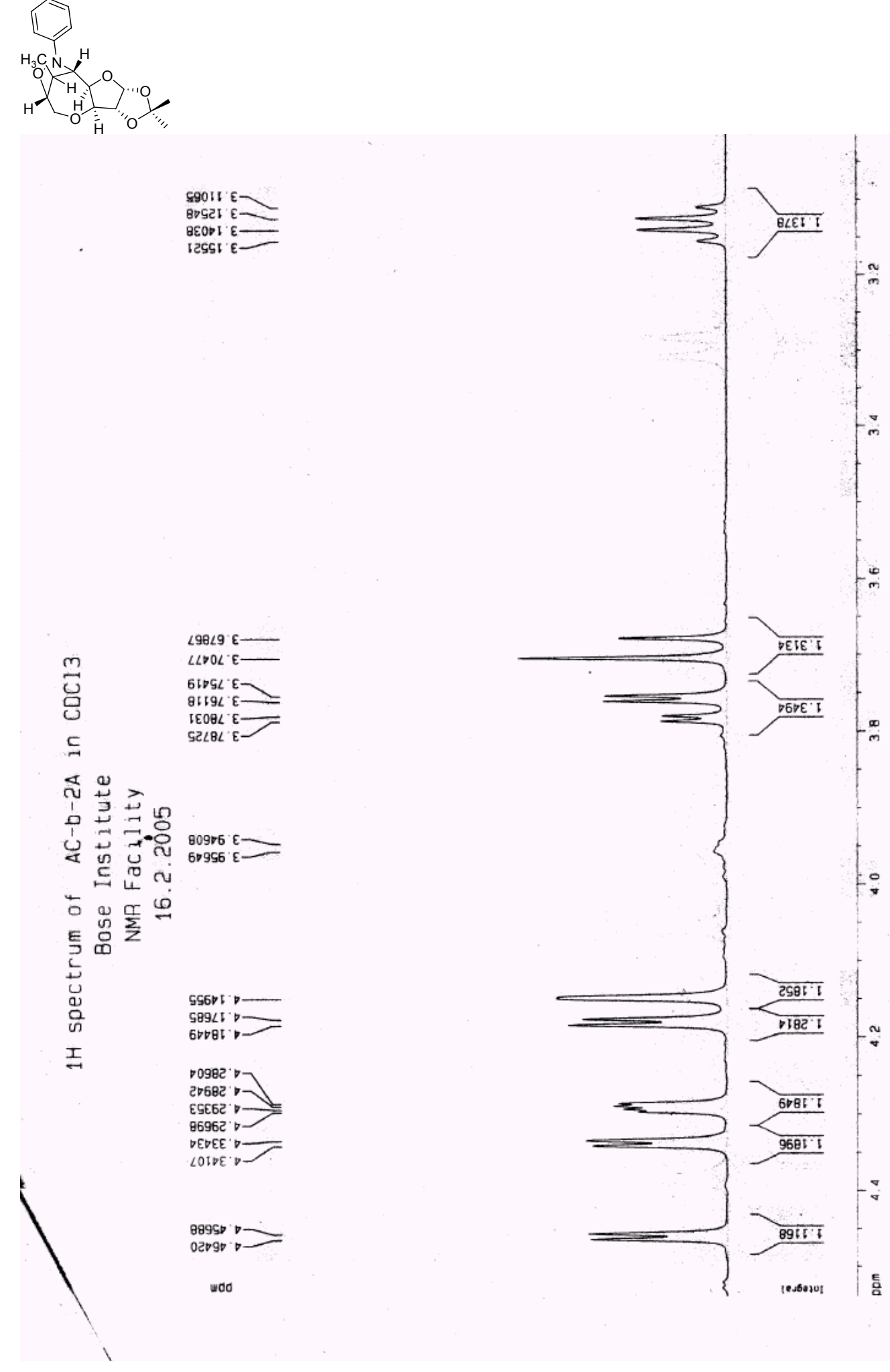

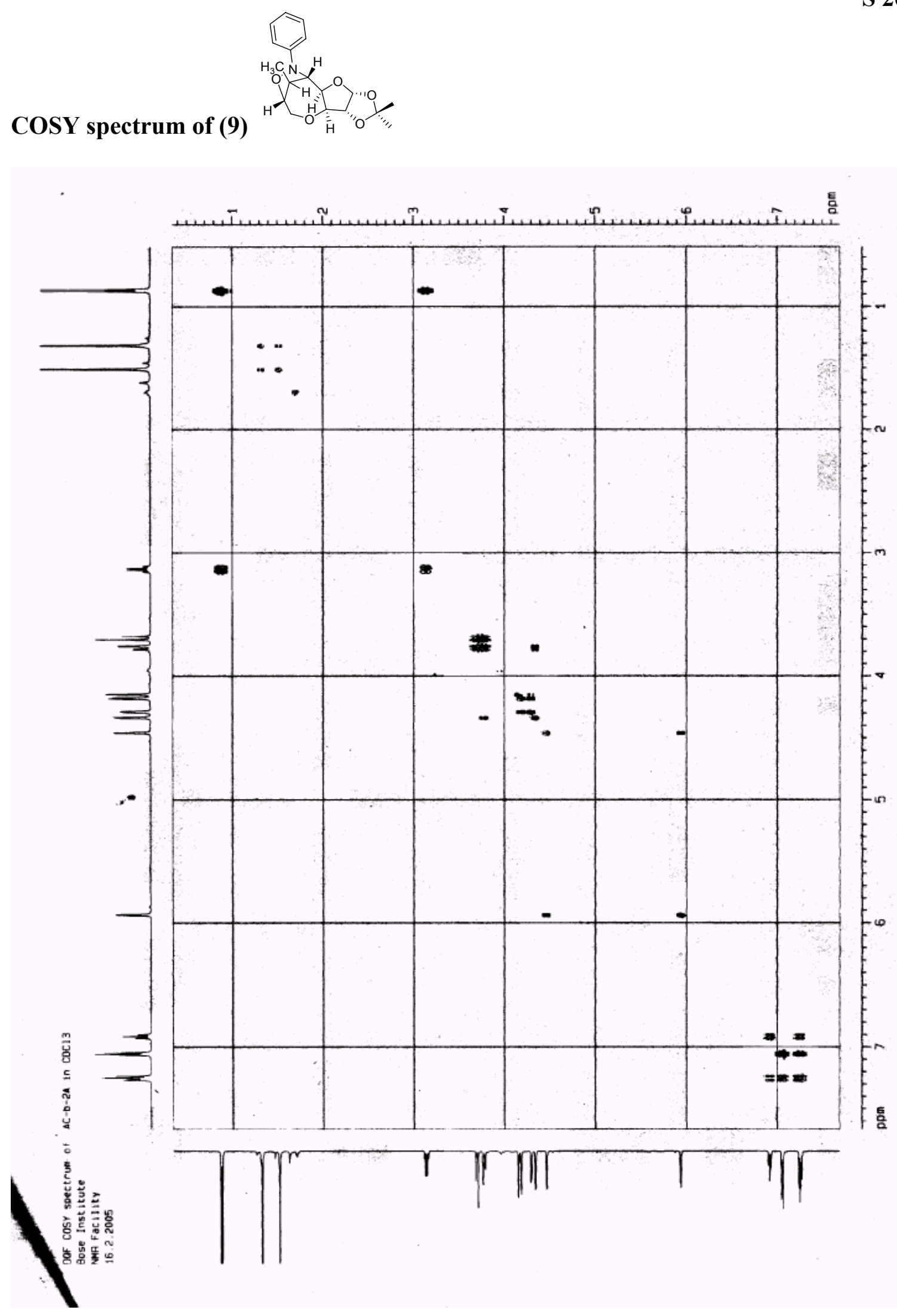


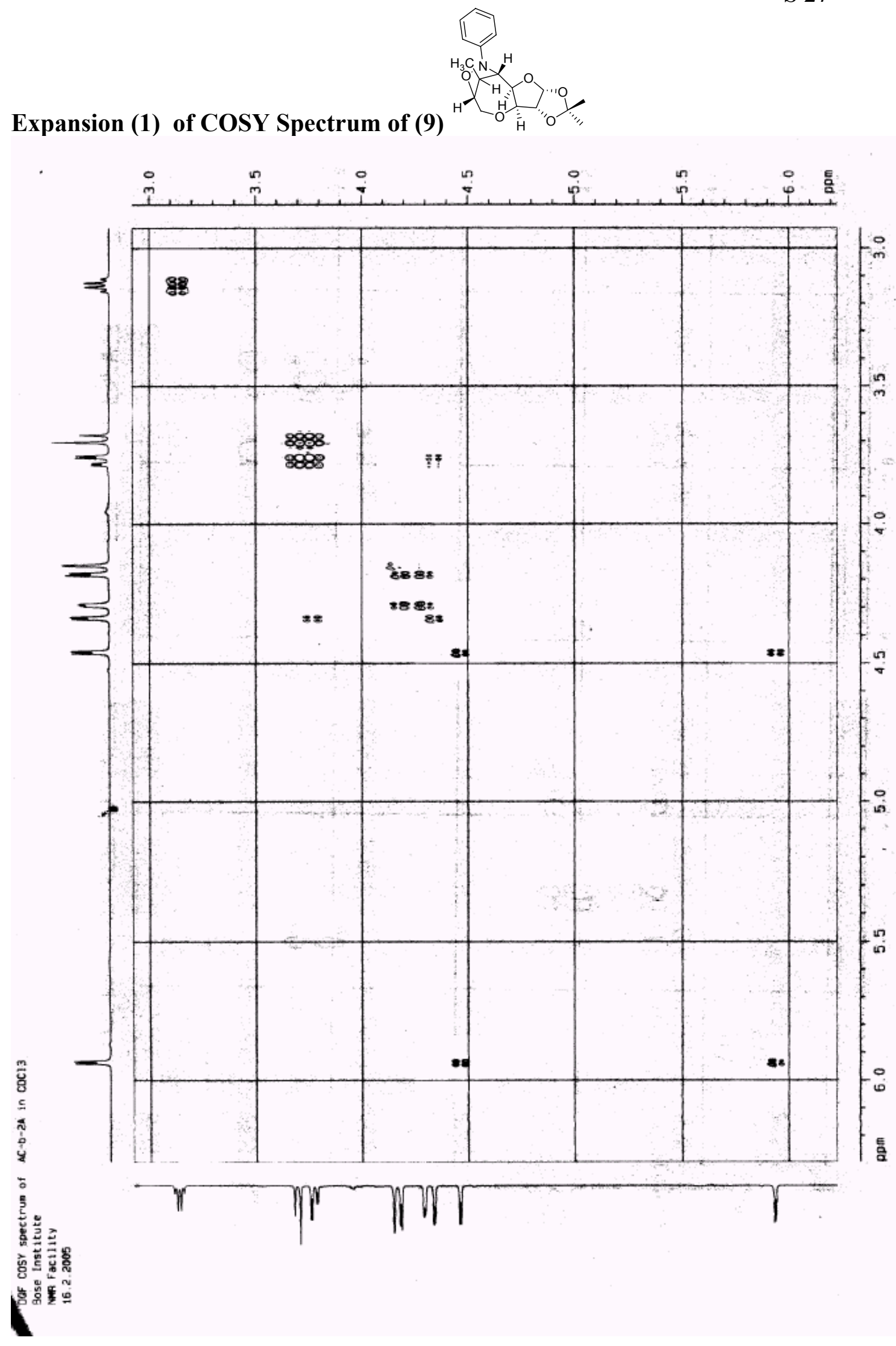



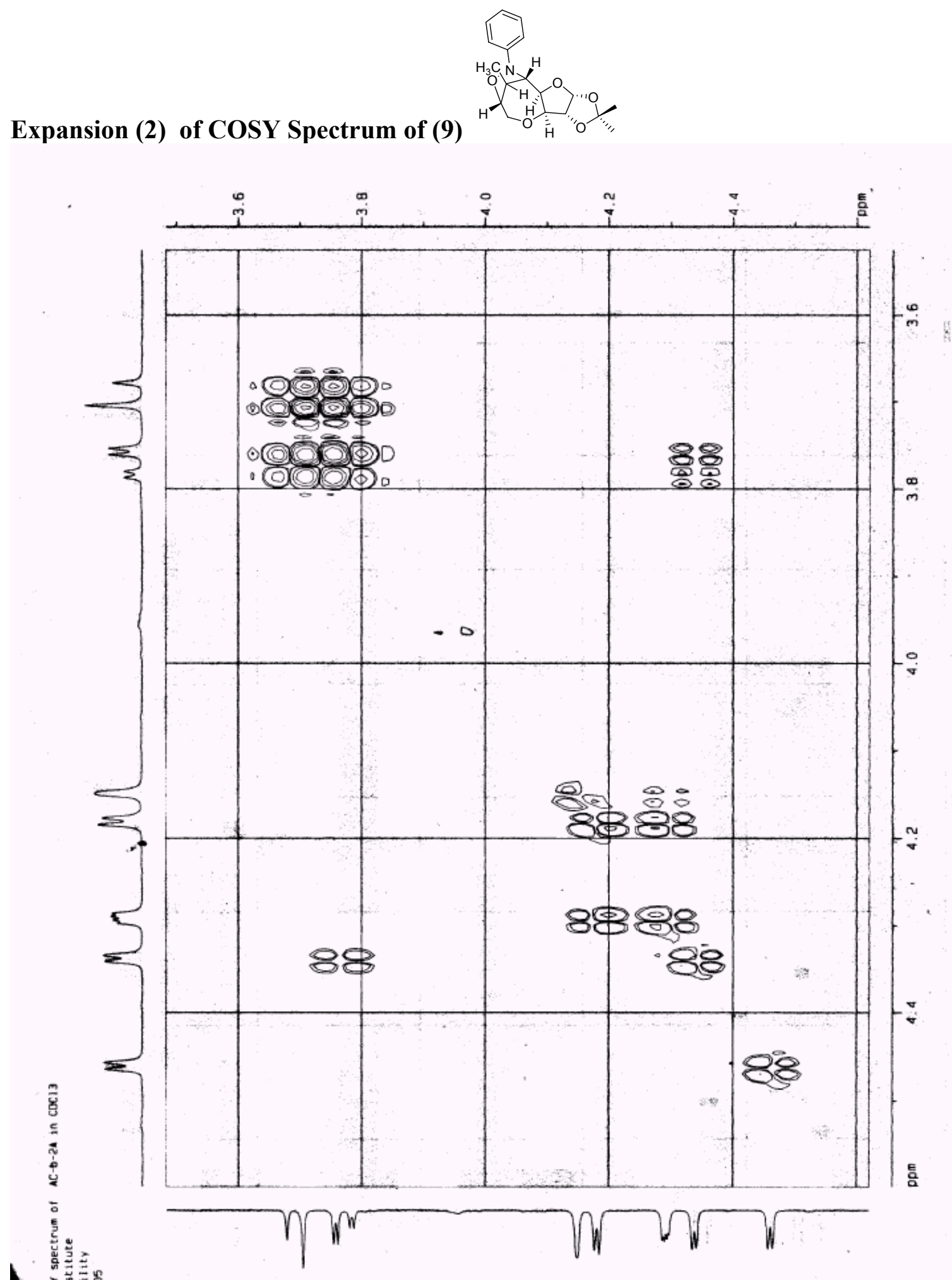

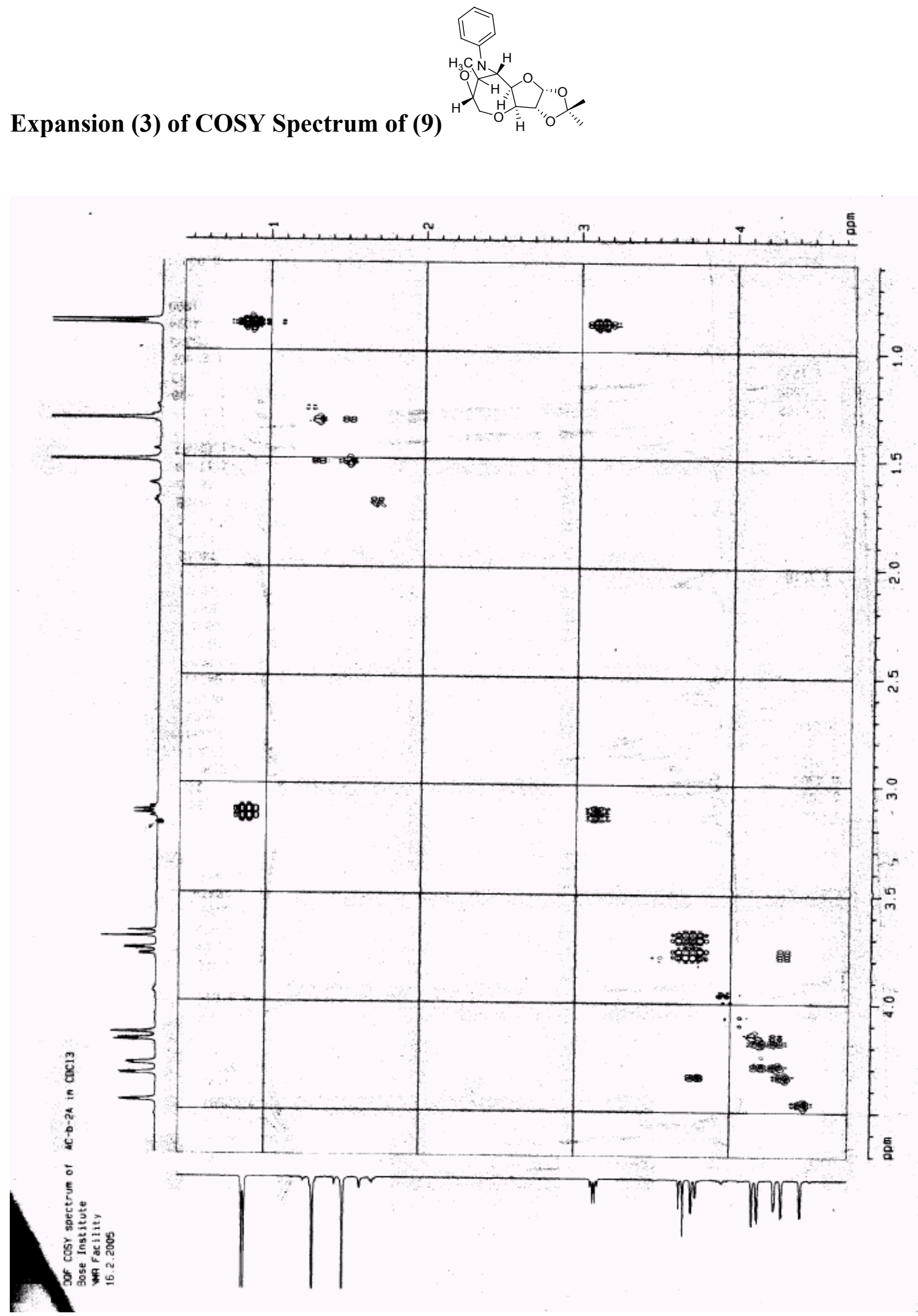

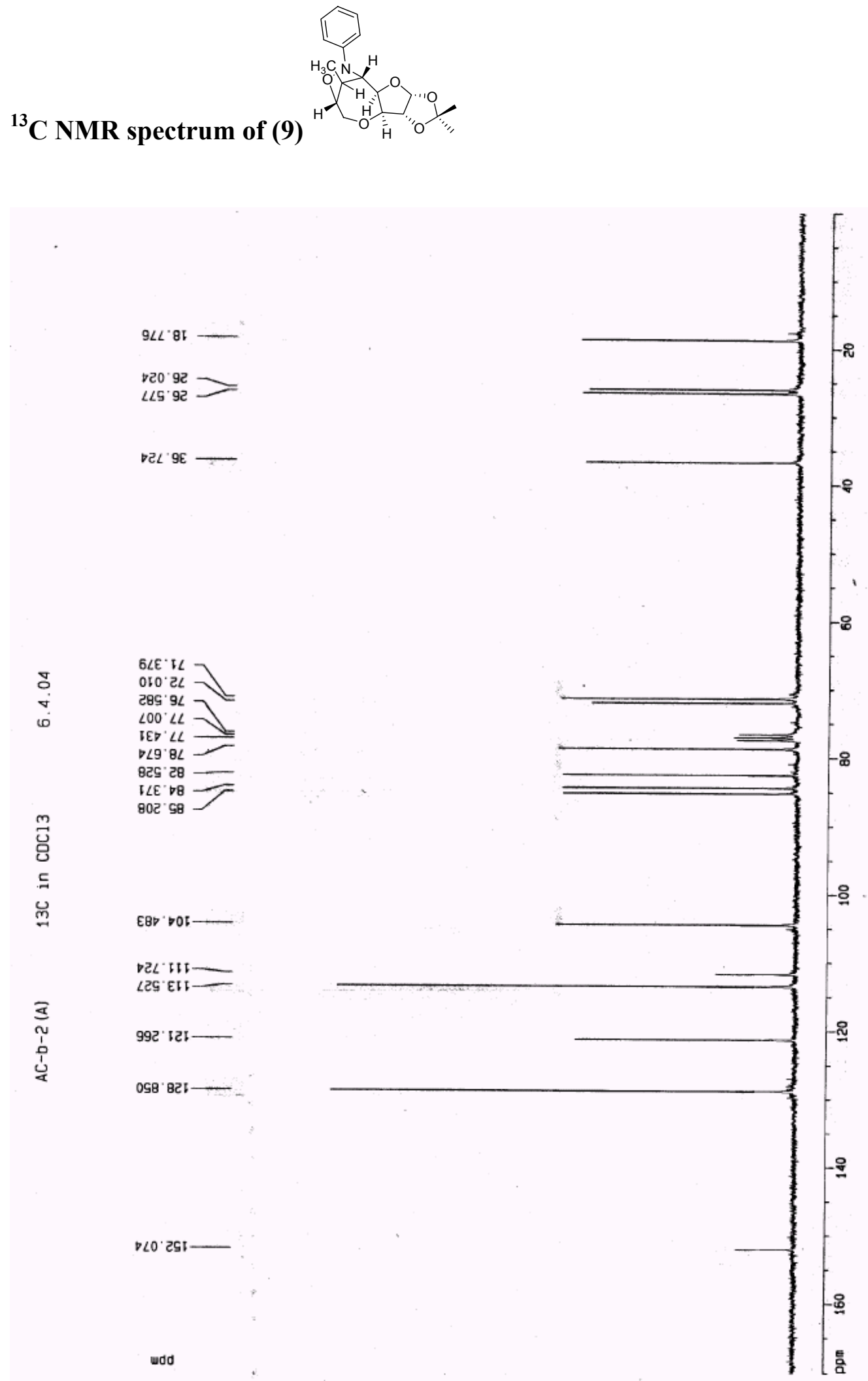


\section{${ }^{1}$ H NMR spectrum of (10)}
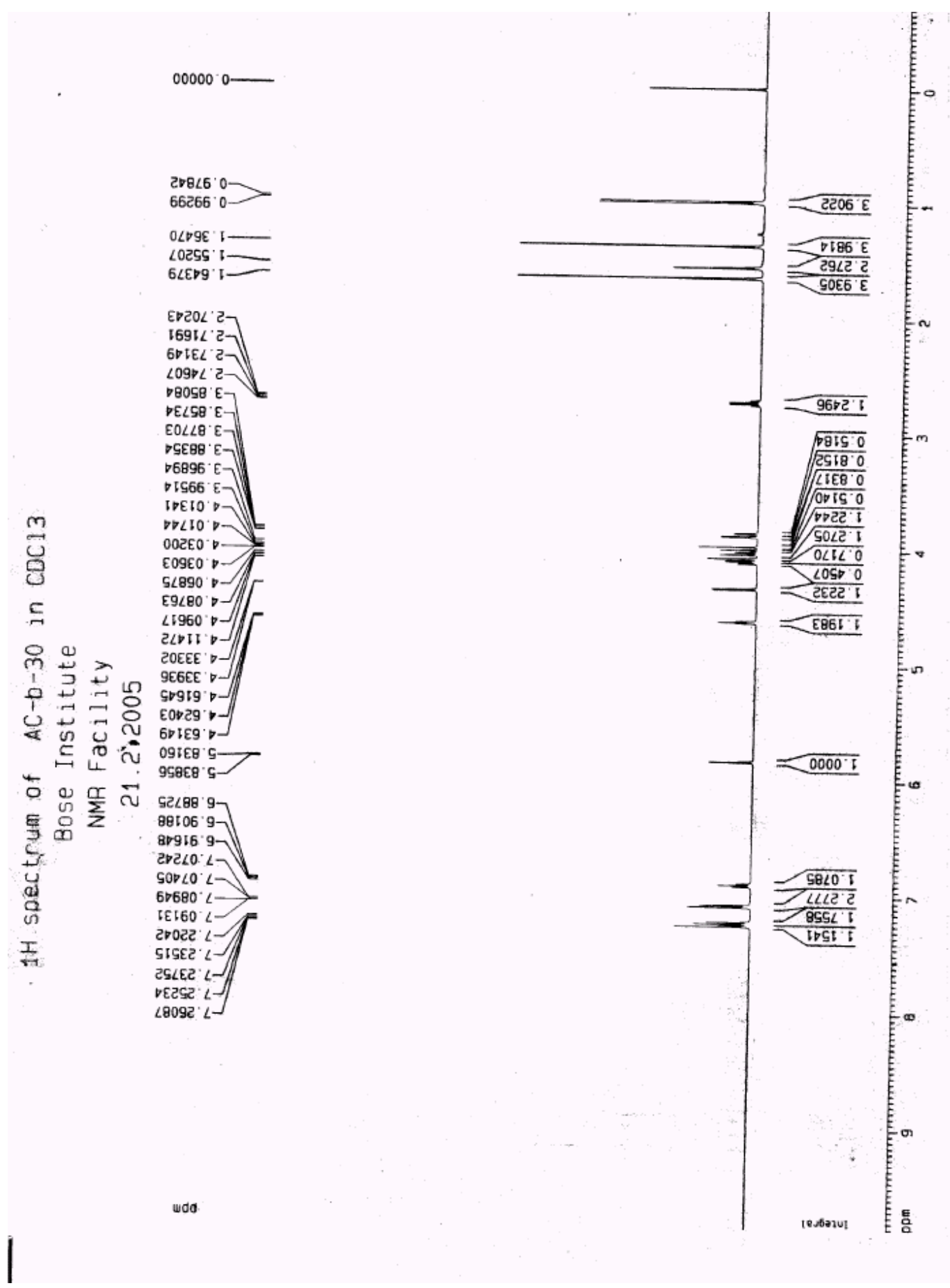

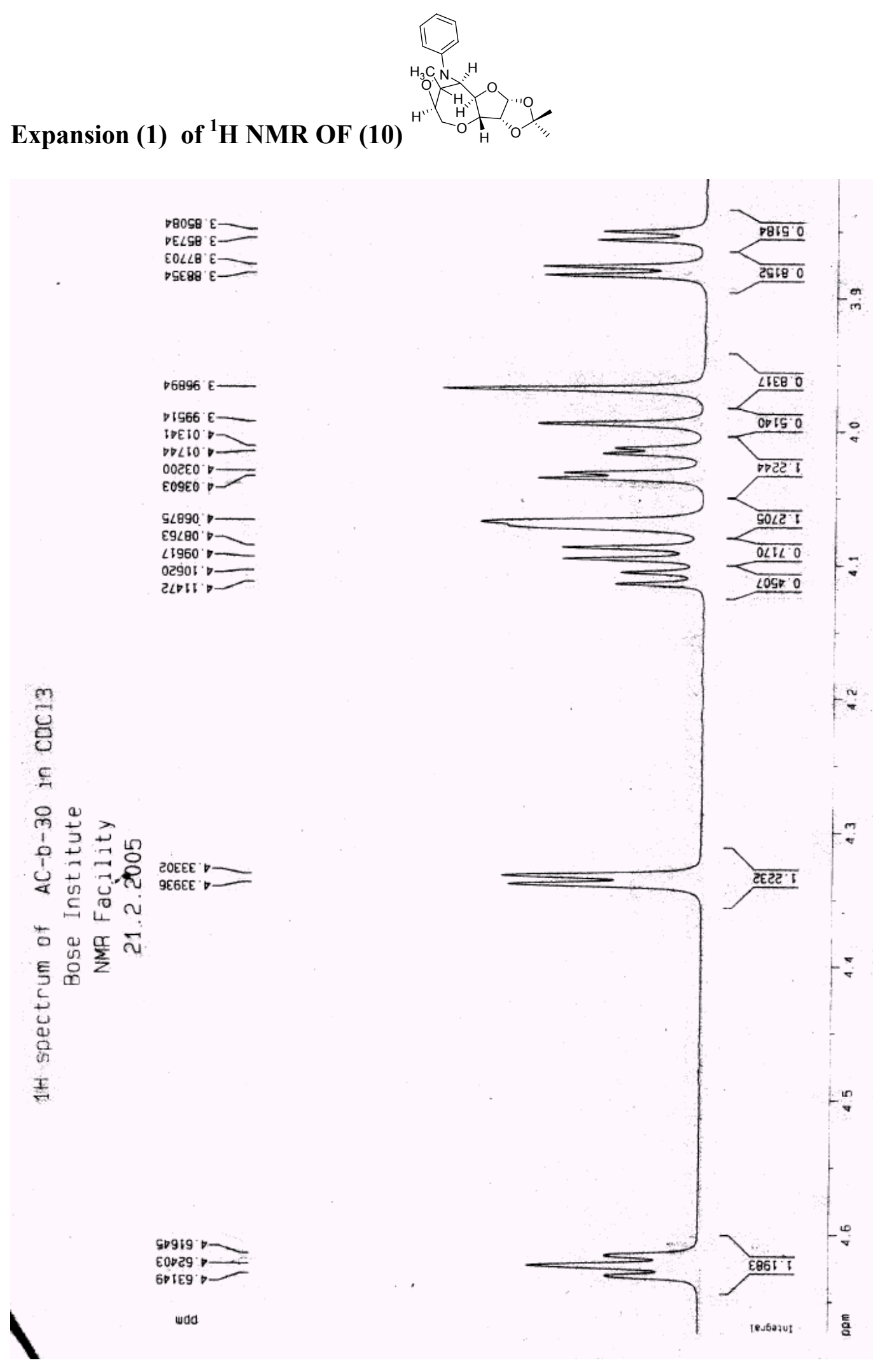

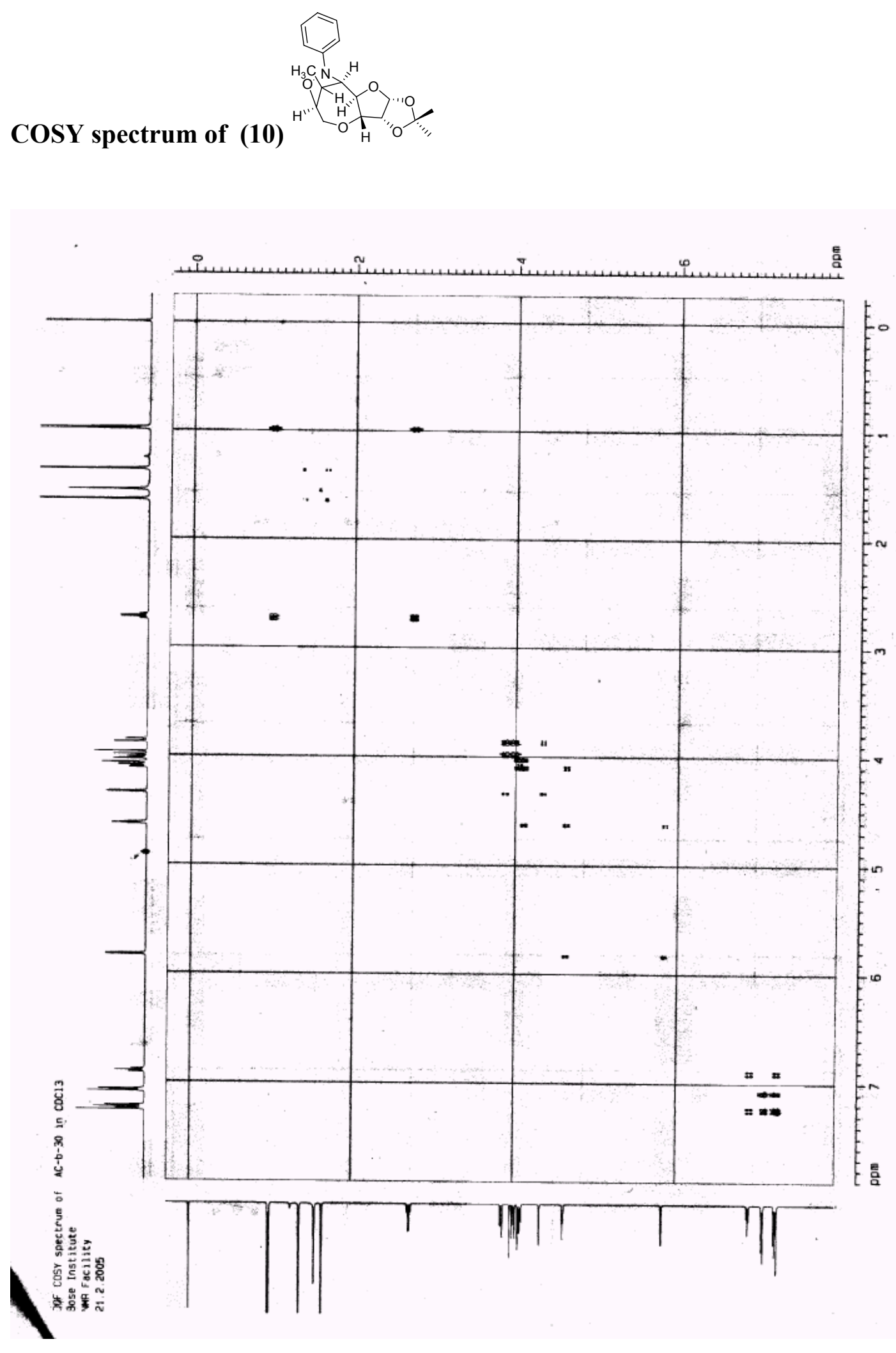

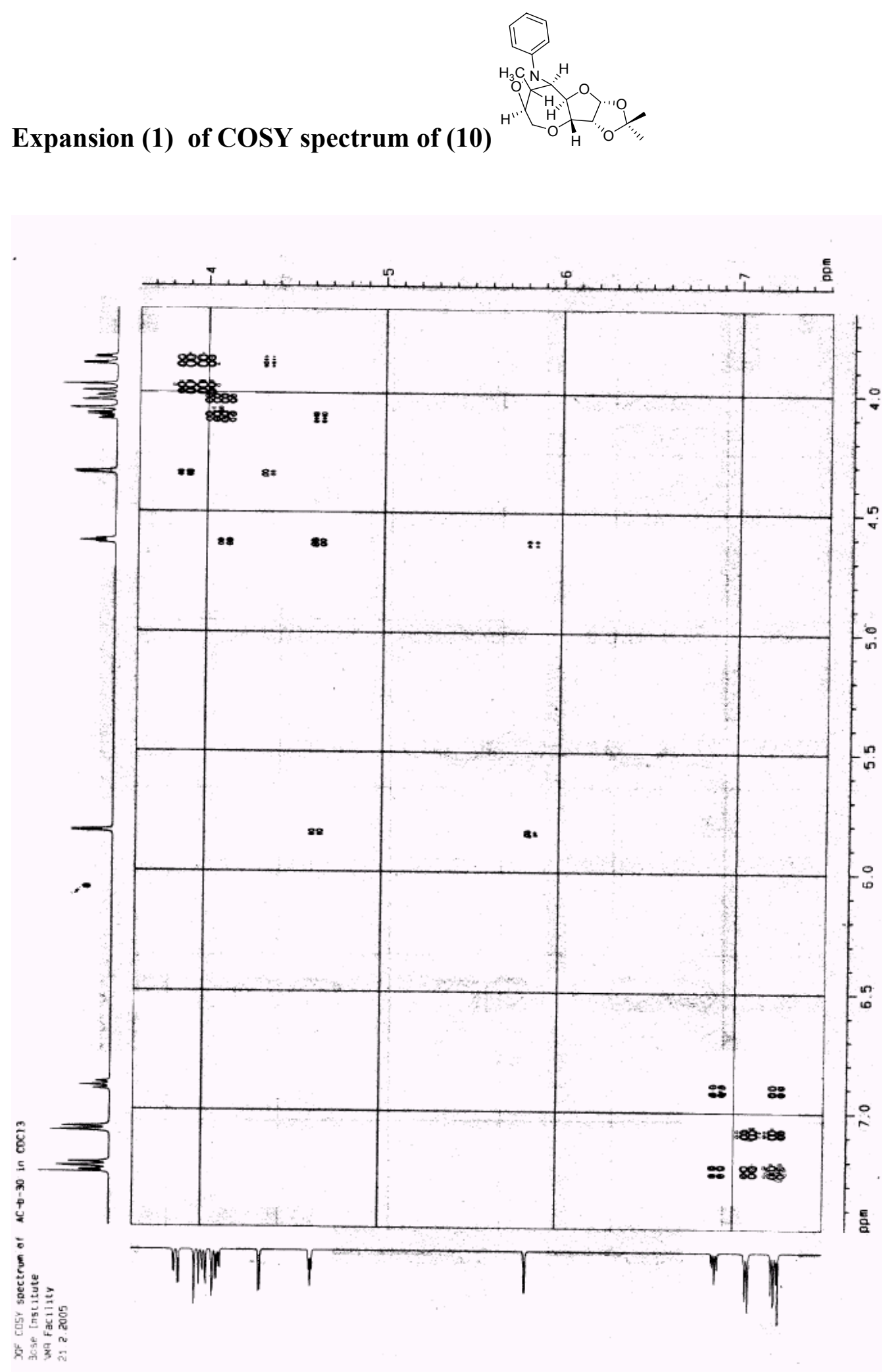
Expansion (2) of COSY spectrum of (10)

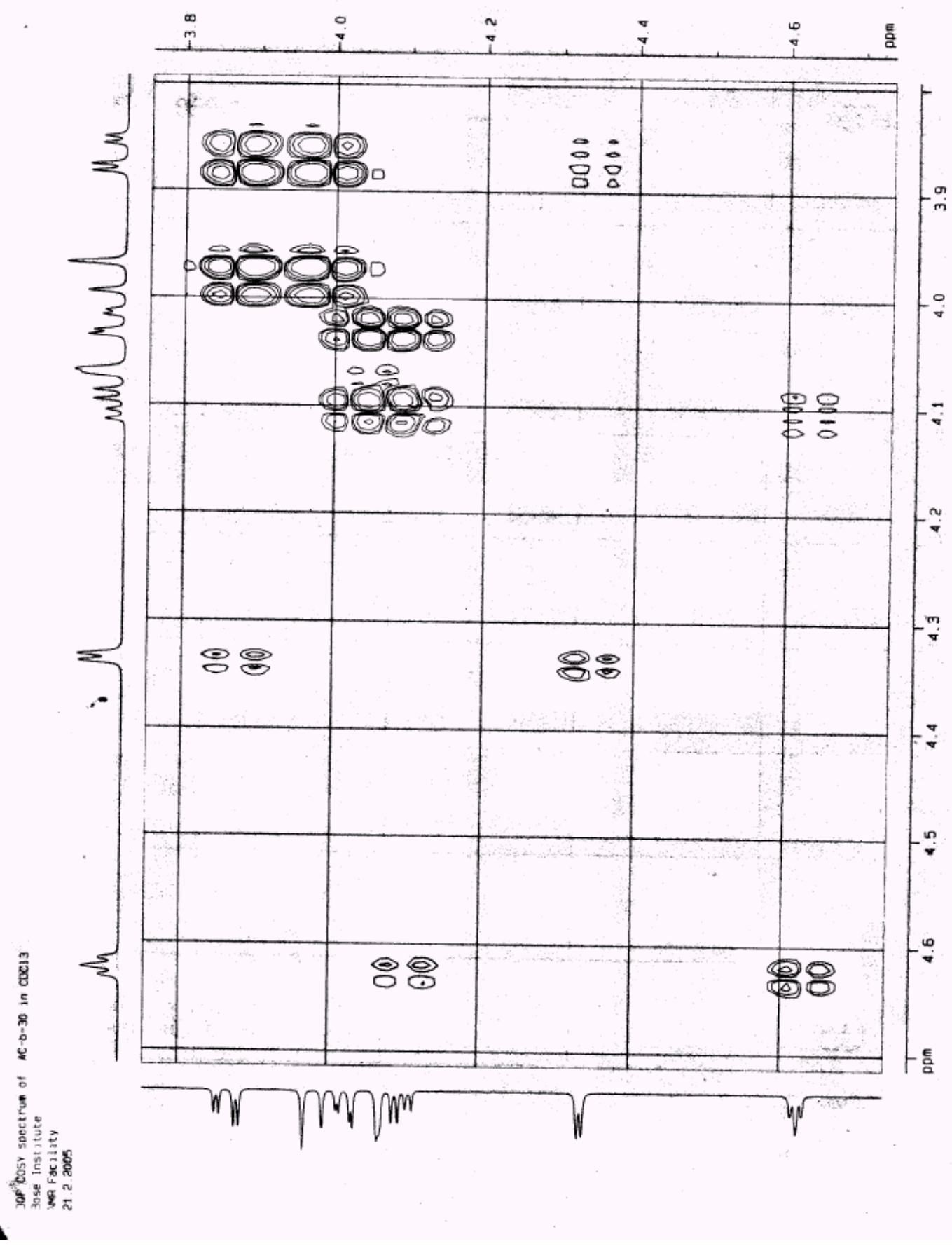



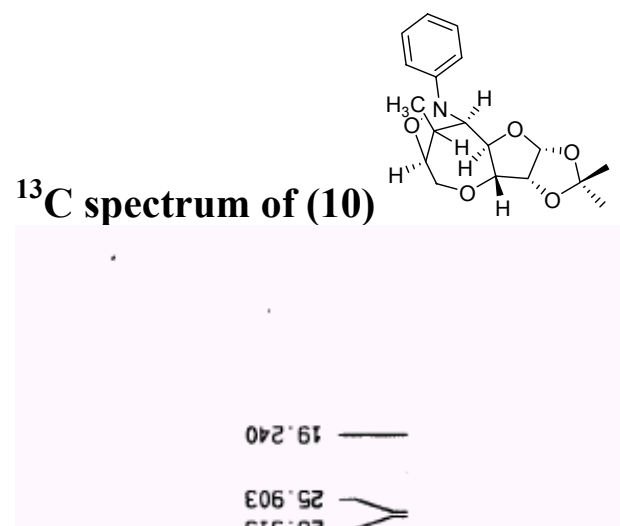

GIE. $95>$

oเร. $6 \varepsilon$
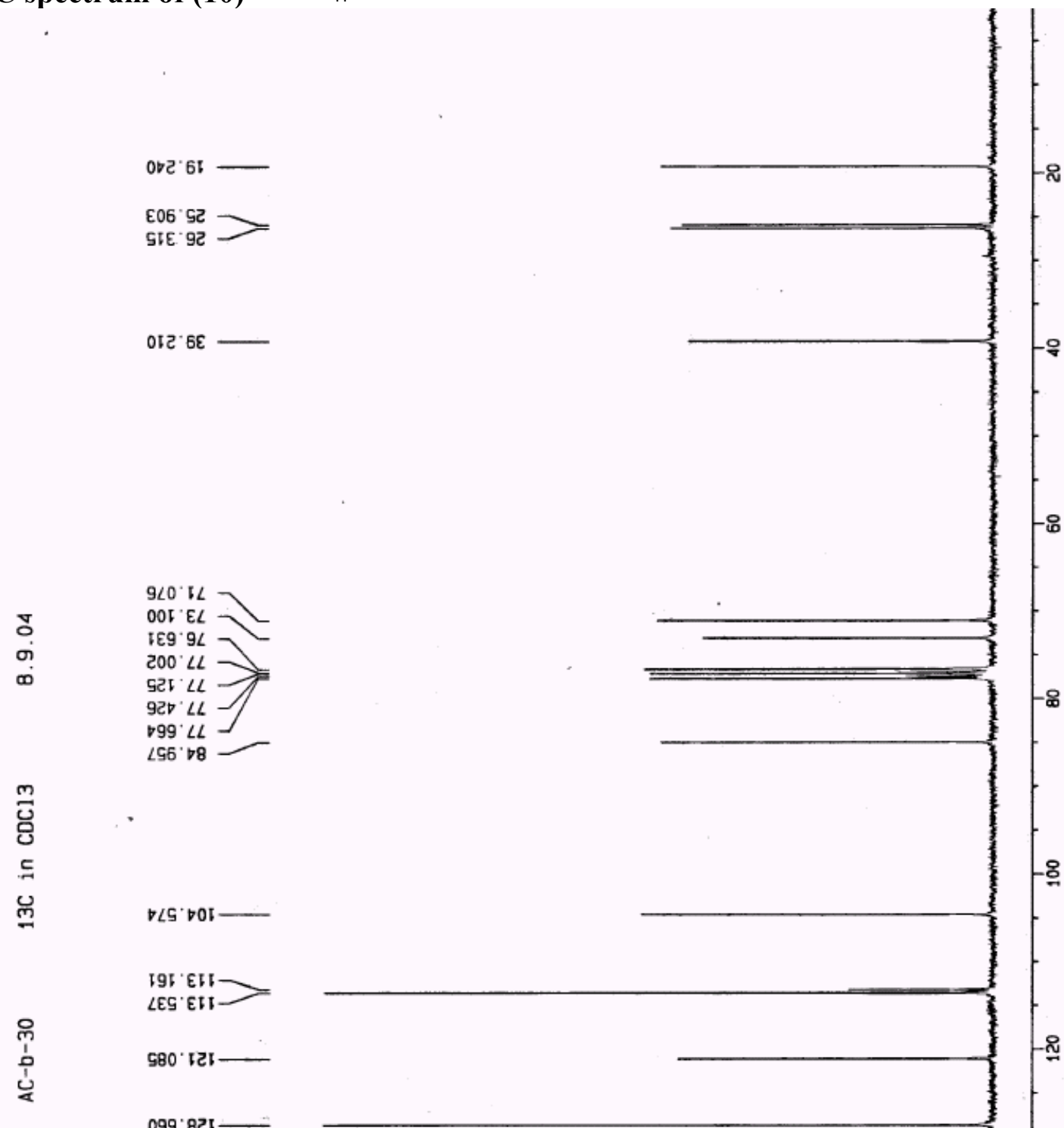

$099^{\prime} 821$

$969^{*} \mathrm{2St}$

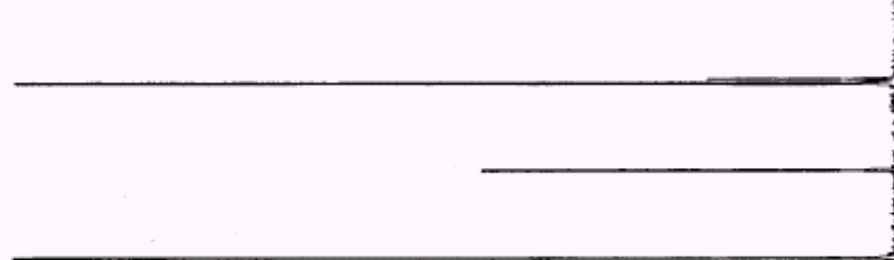




\section{${ }^{1}$ H NMR spectrum of (11)}
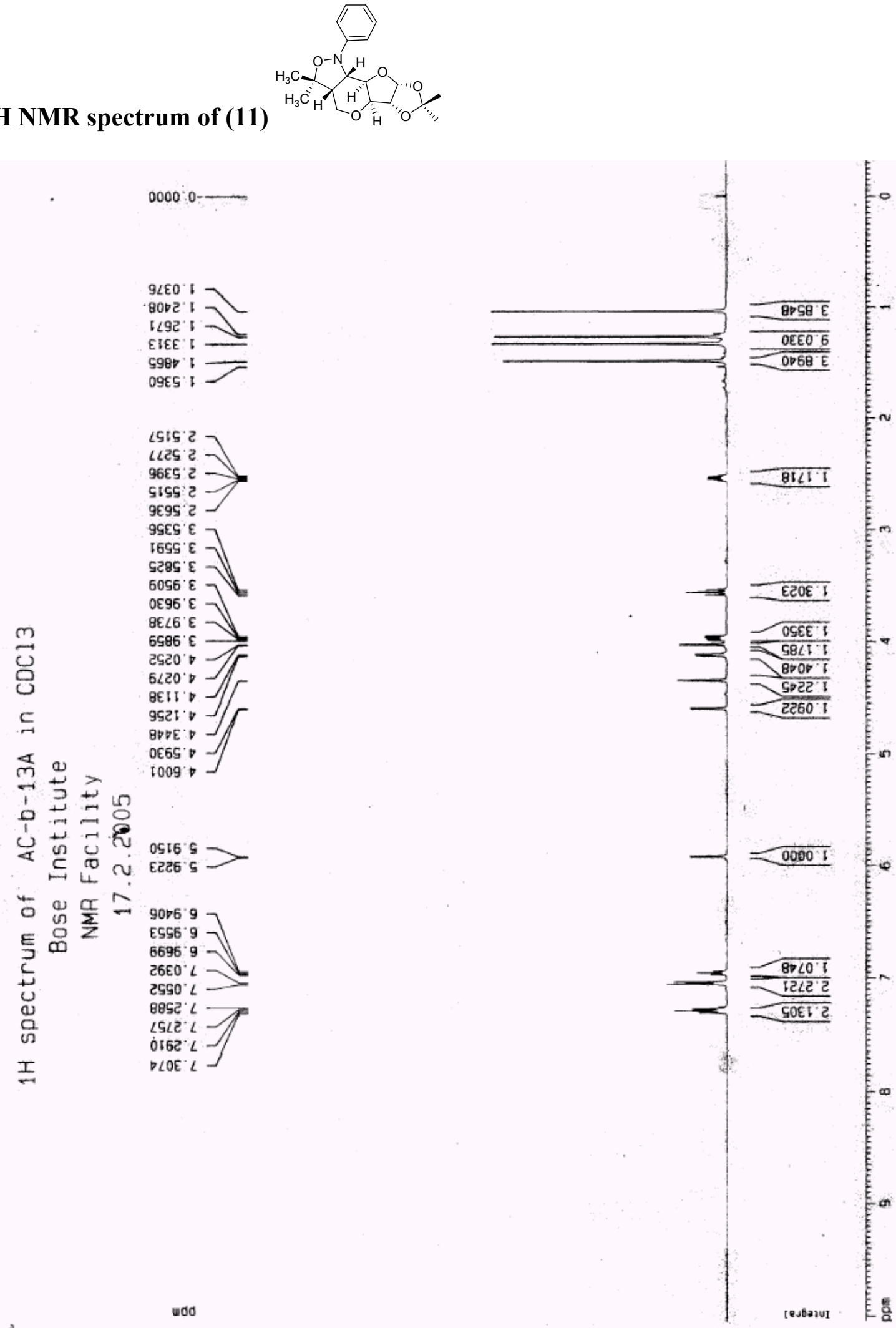
Expansion (1) of ${ }^{1} \mathrm{H}$ NMR spectrum of (11)
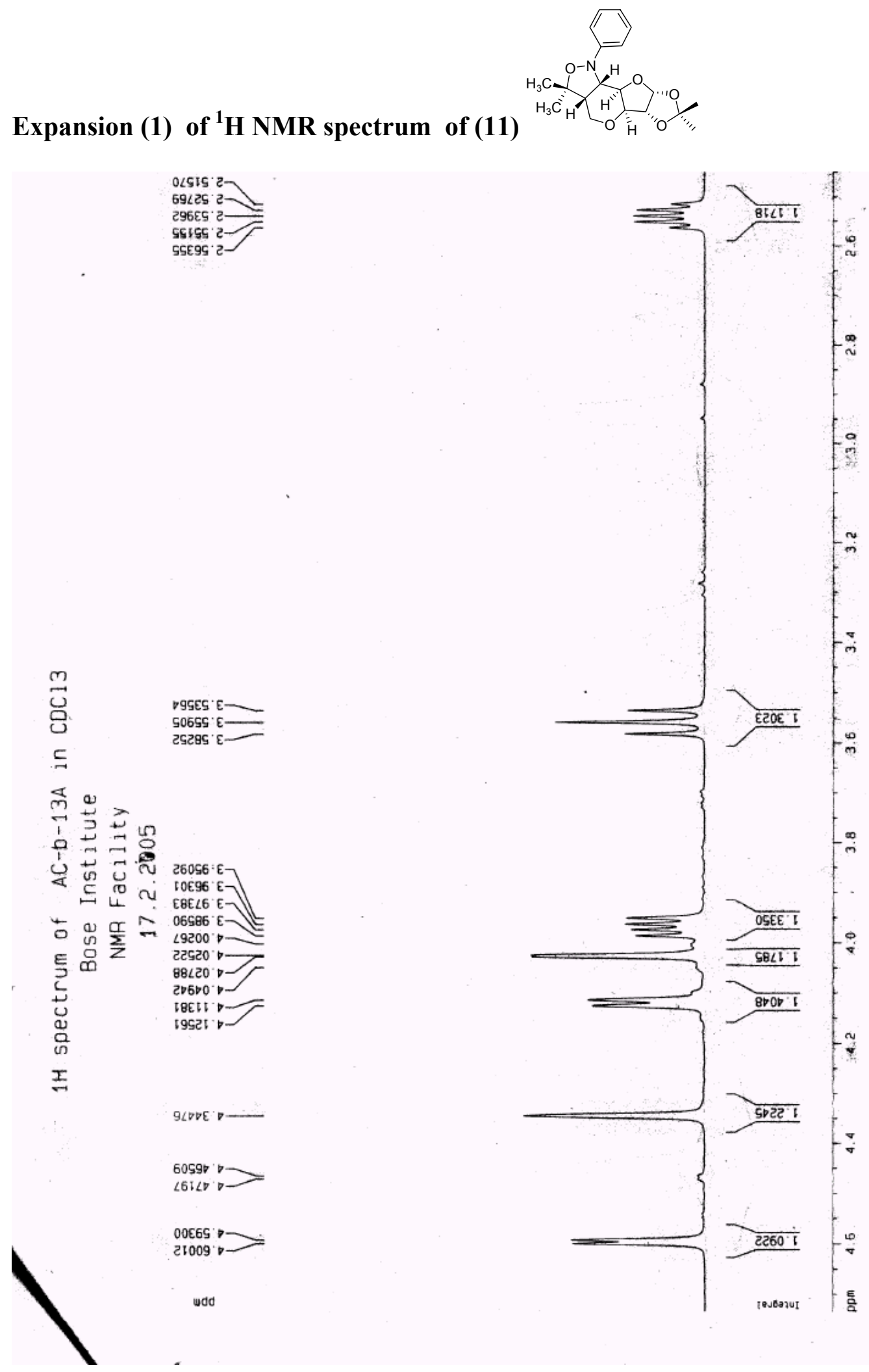
COSY spectrum of (11)

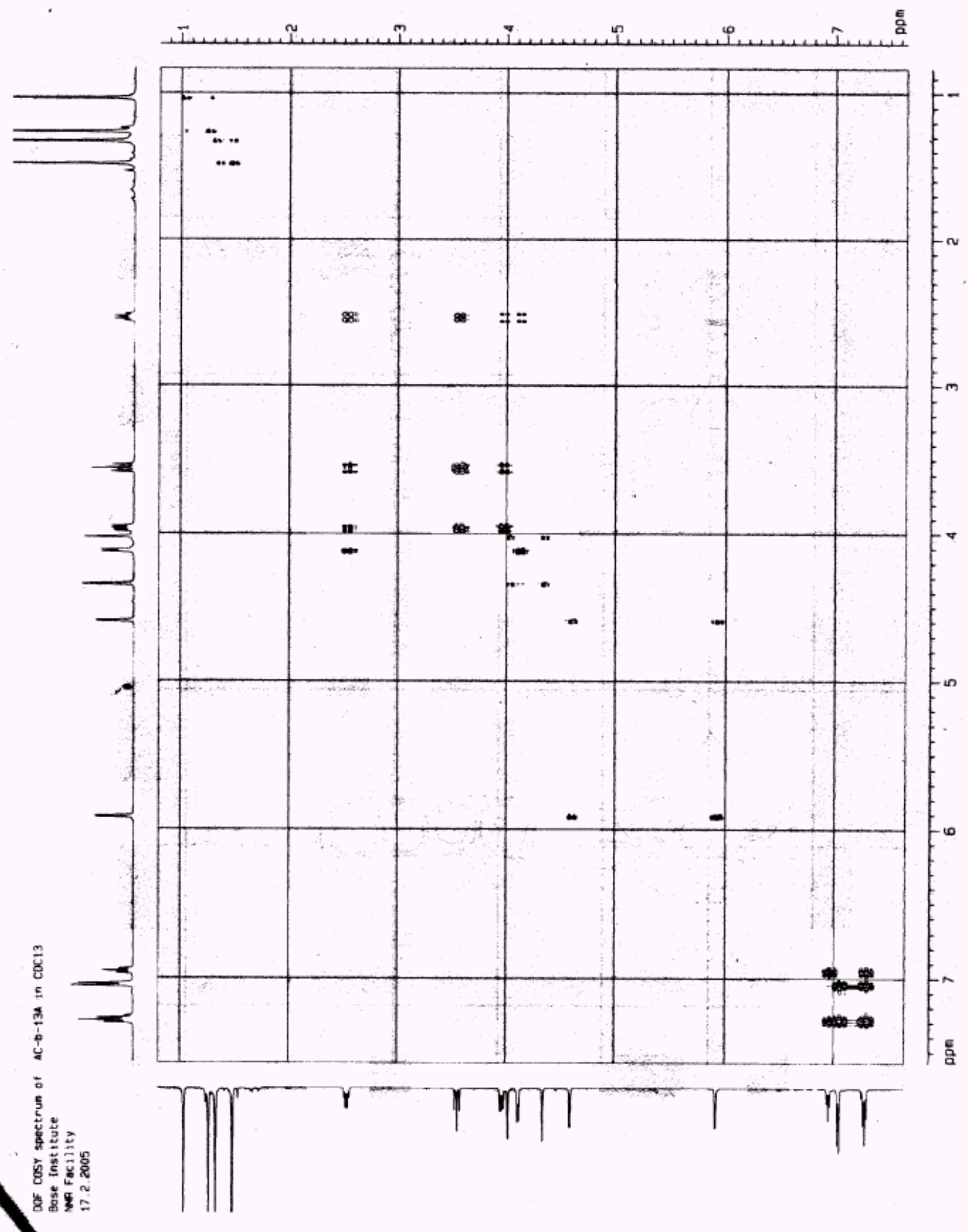


Expansion (1) of COSY spectrum of (11)

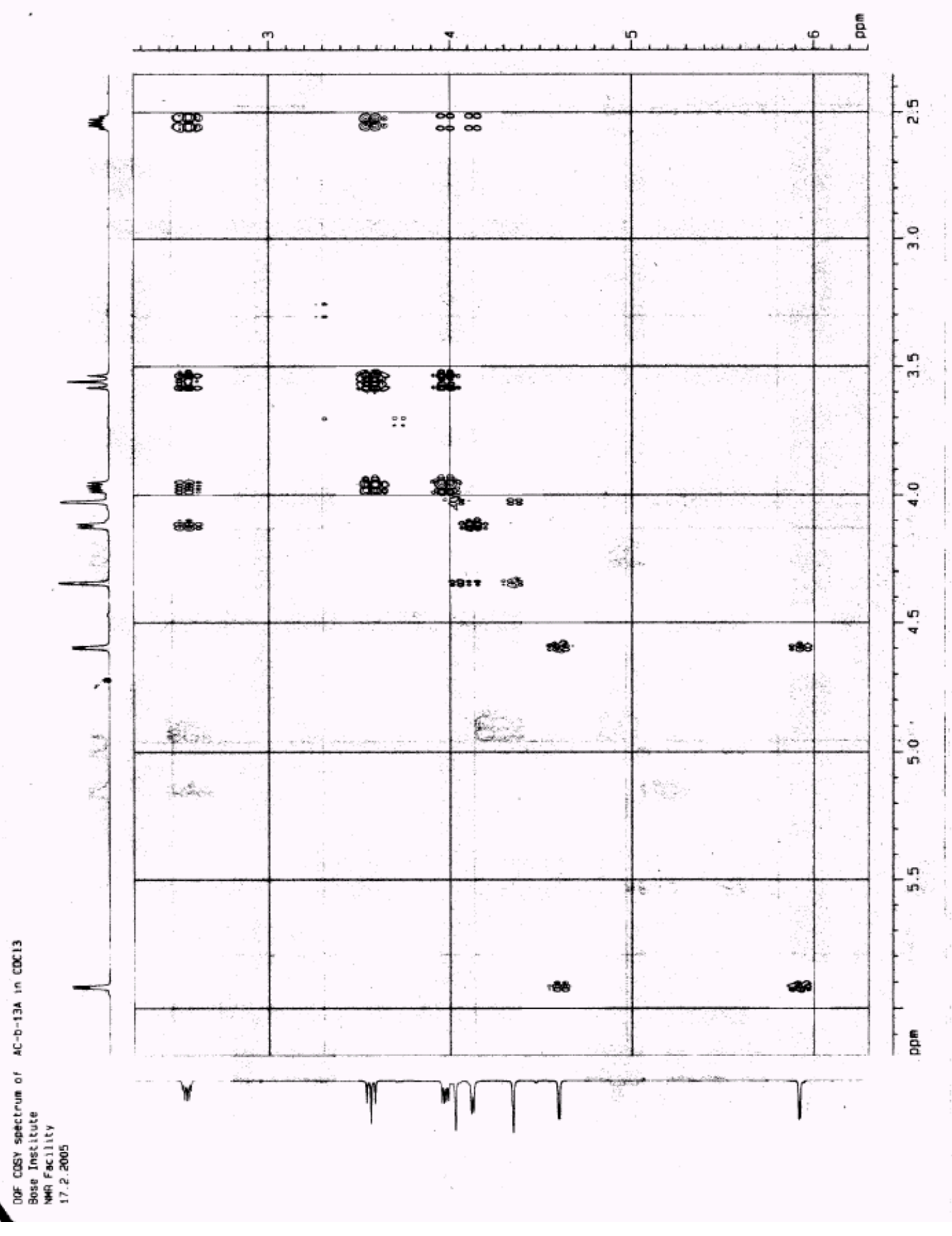




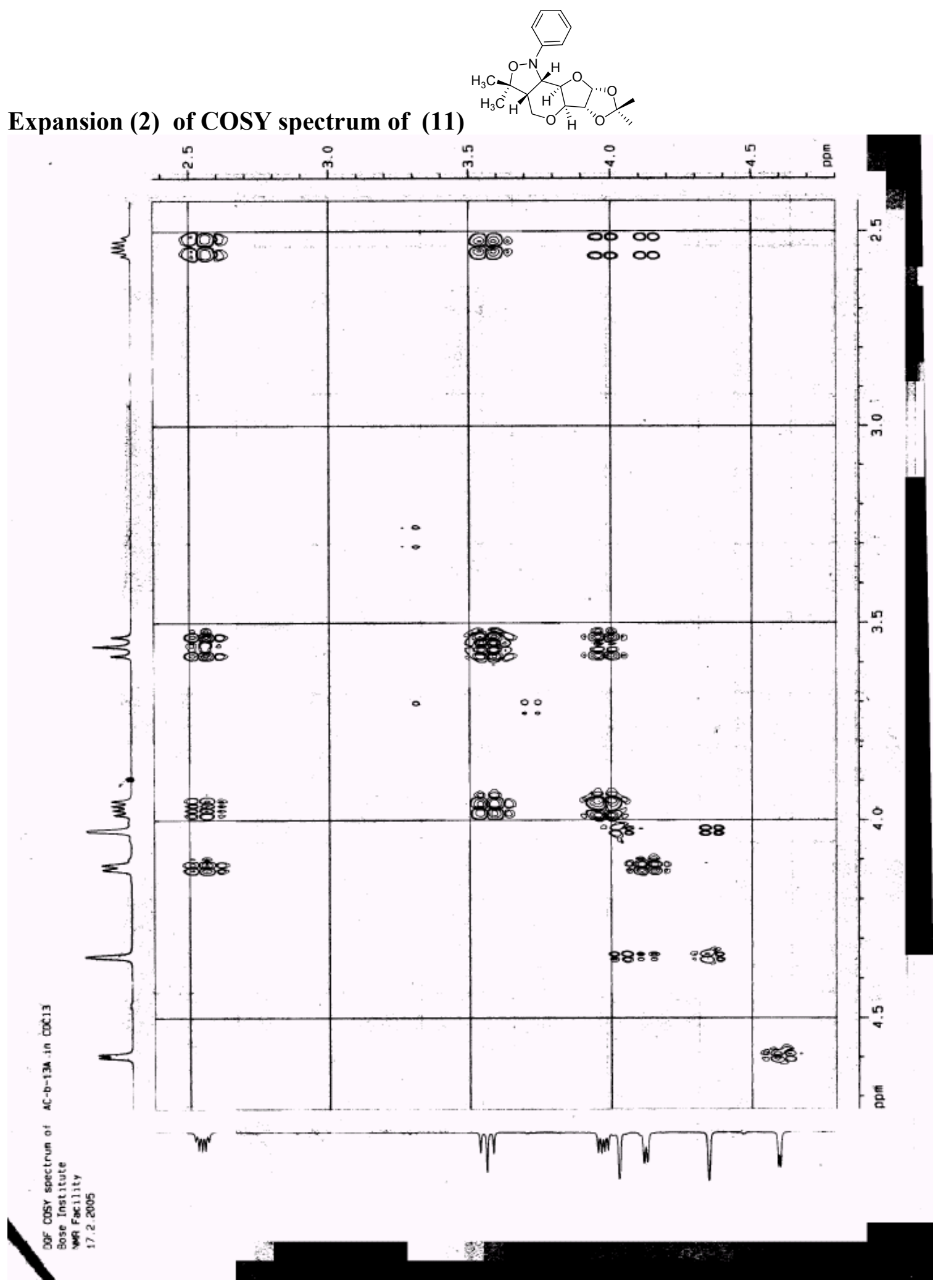




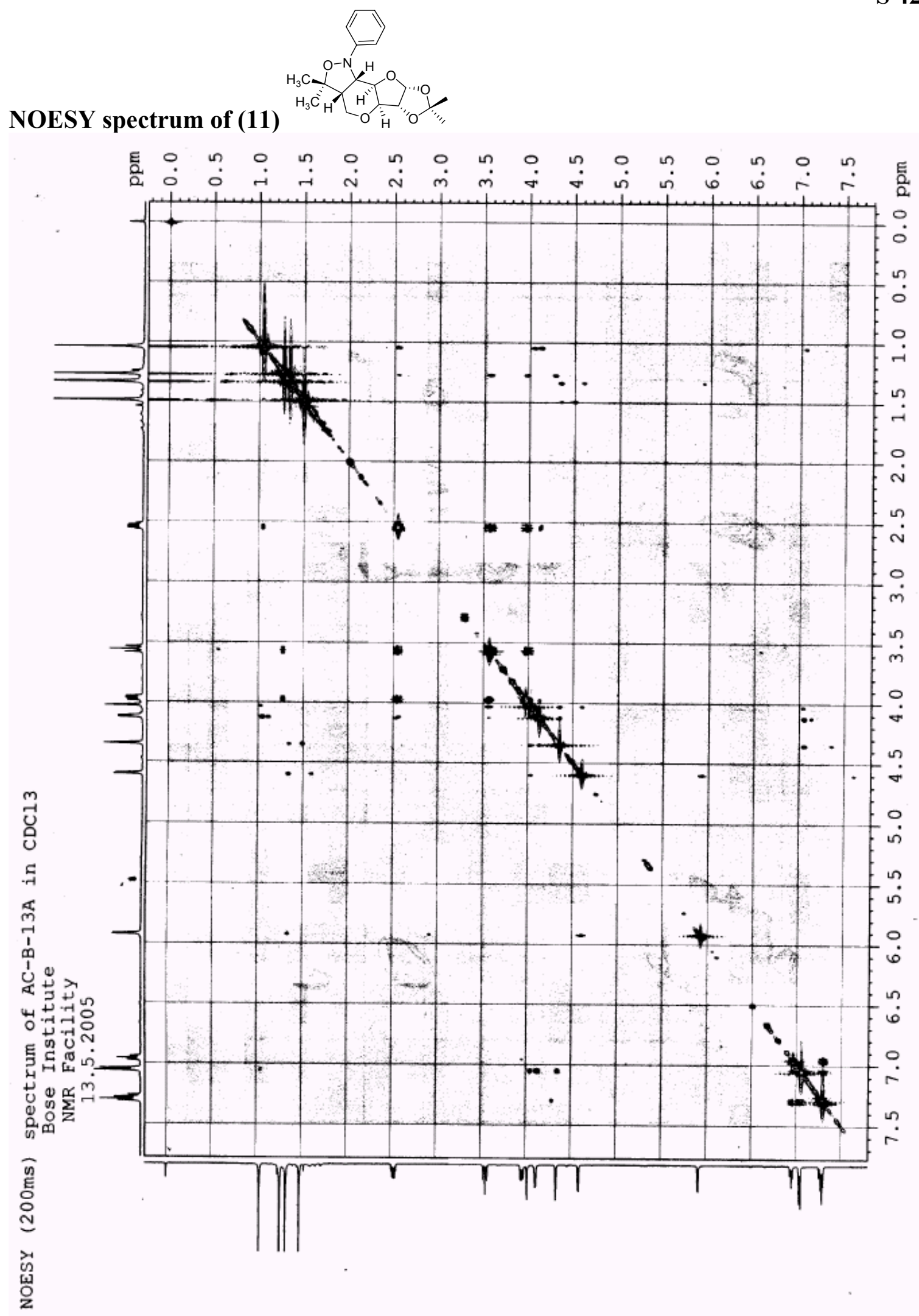


Expansion (1) of NOESY spectrum of (11)

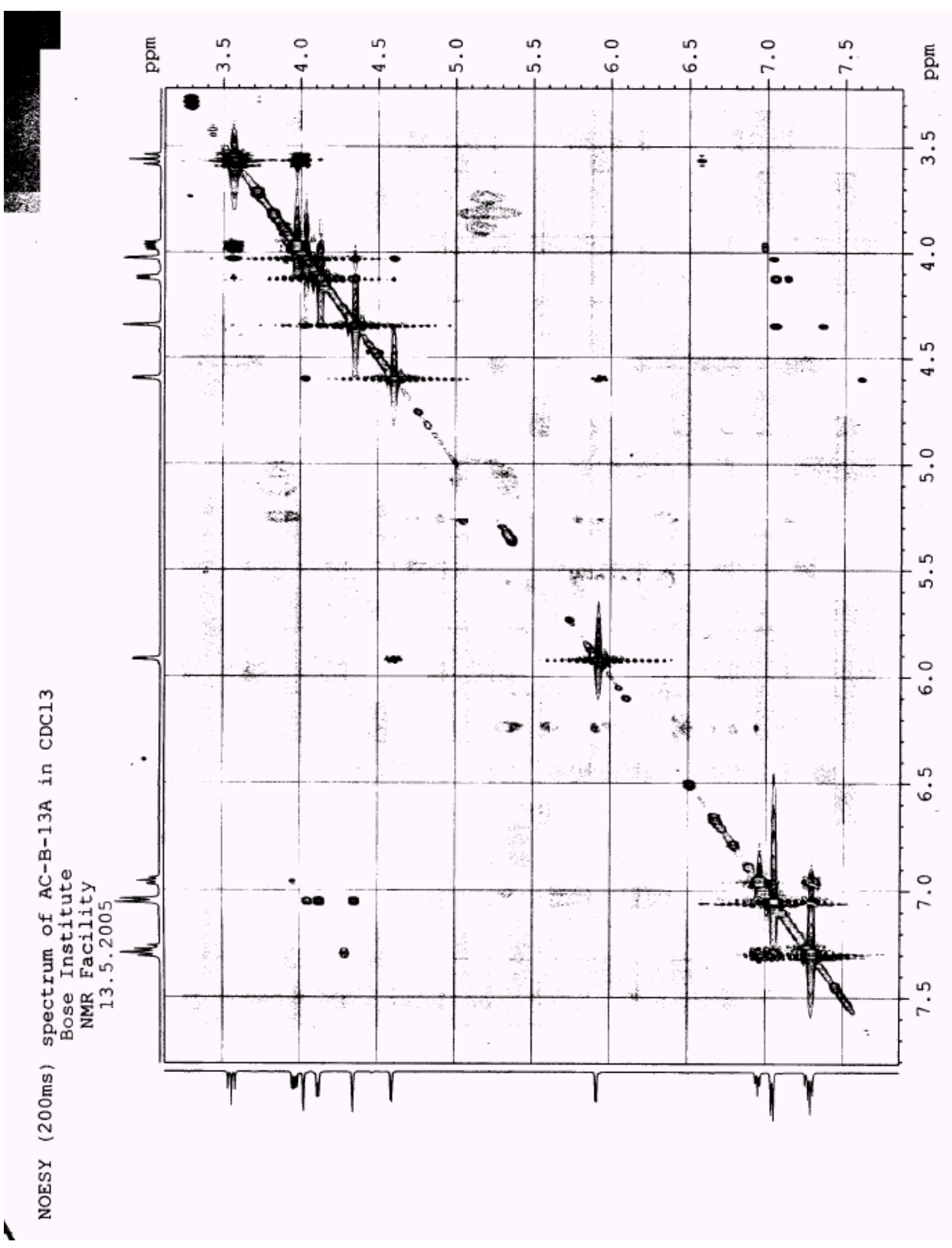


Expansion (2) of NOESY spectrum of (11)

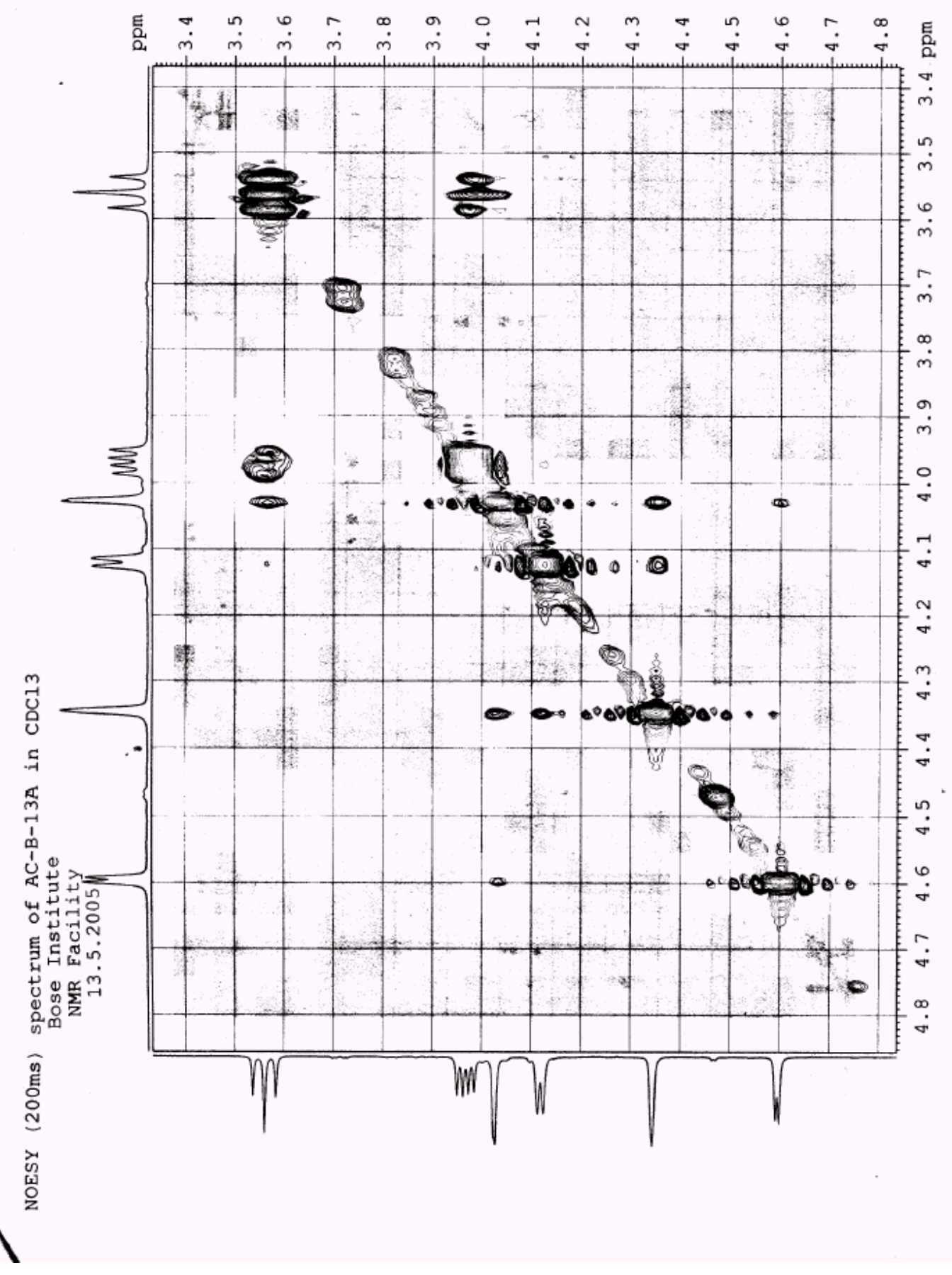


Expansion (3) of NOESY spectrum of (11)

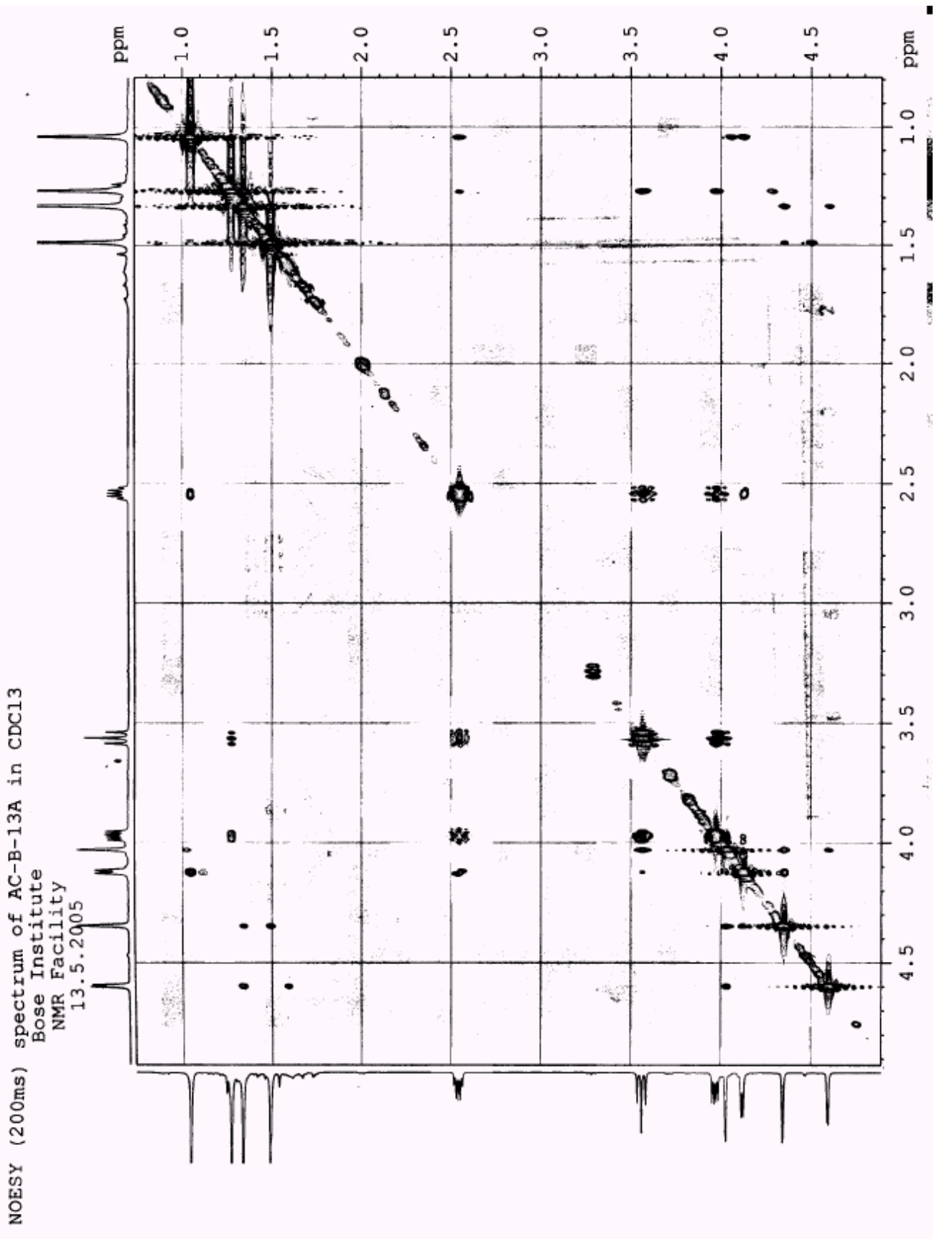



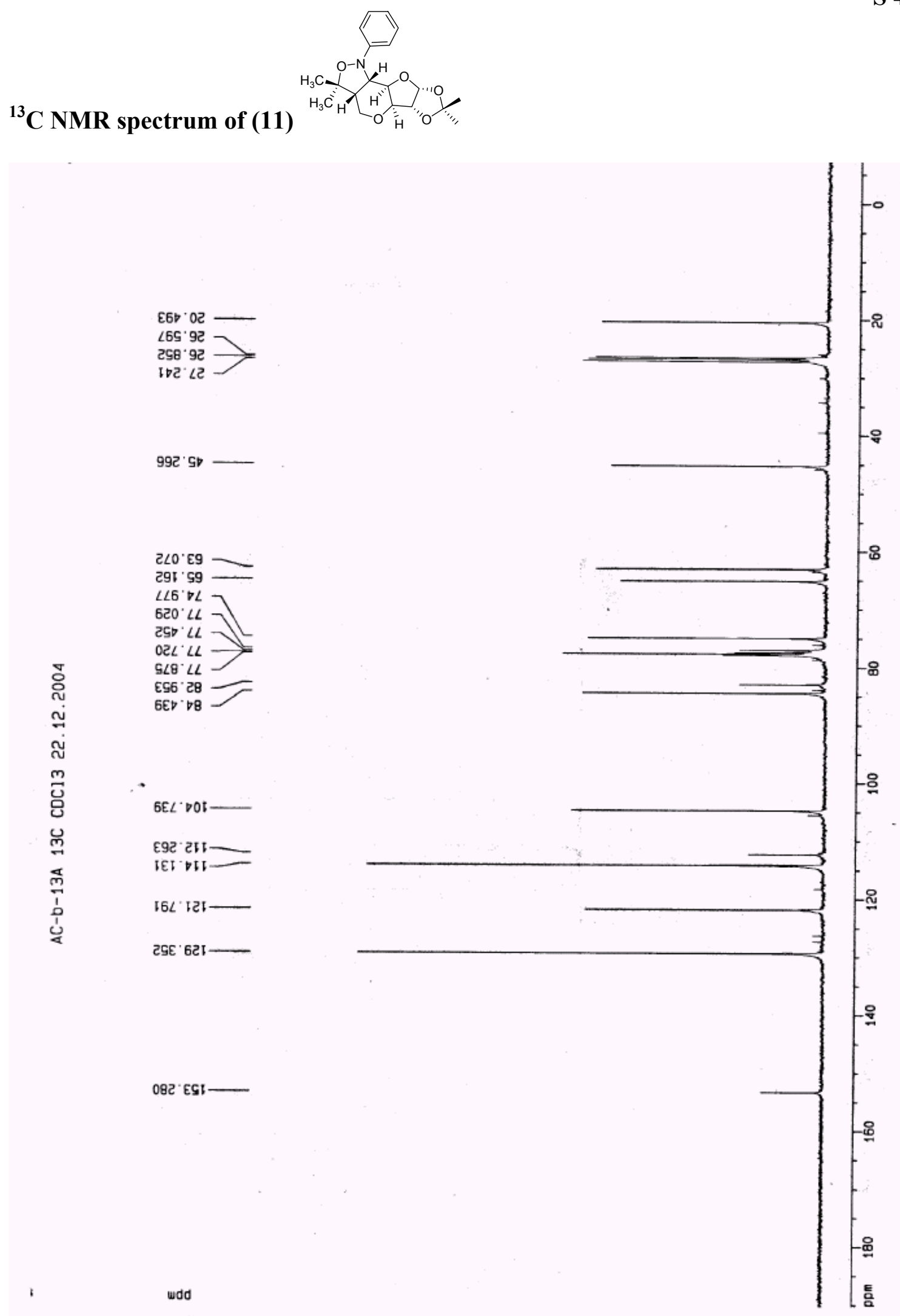

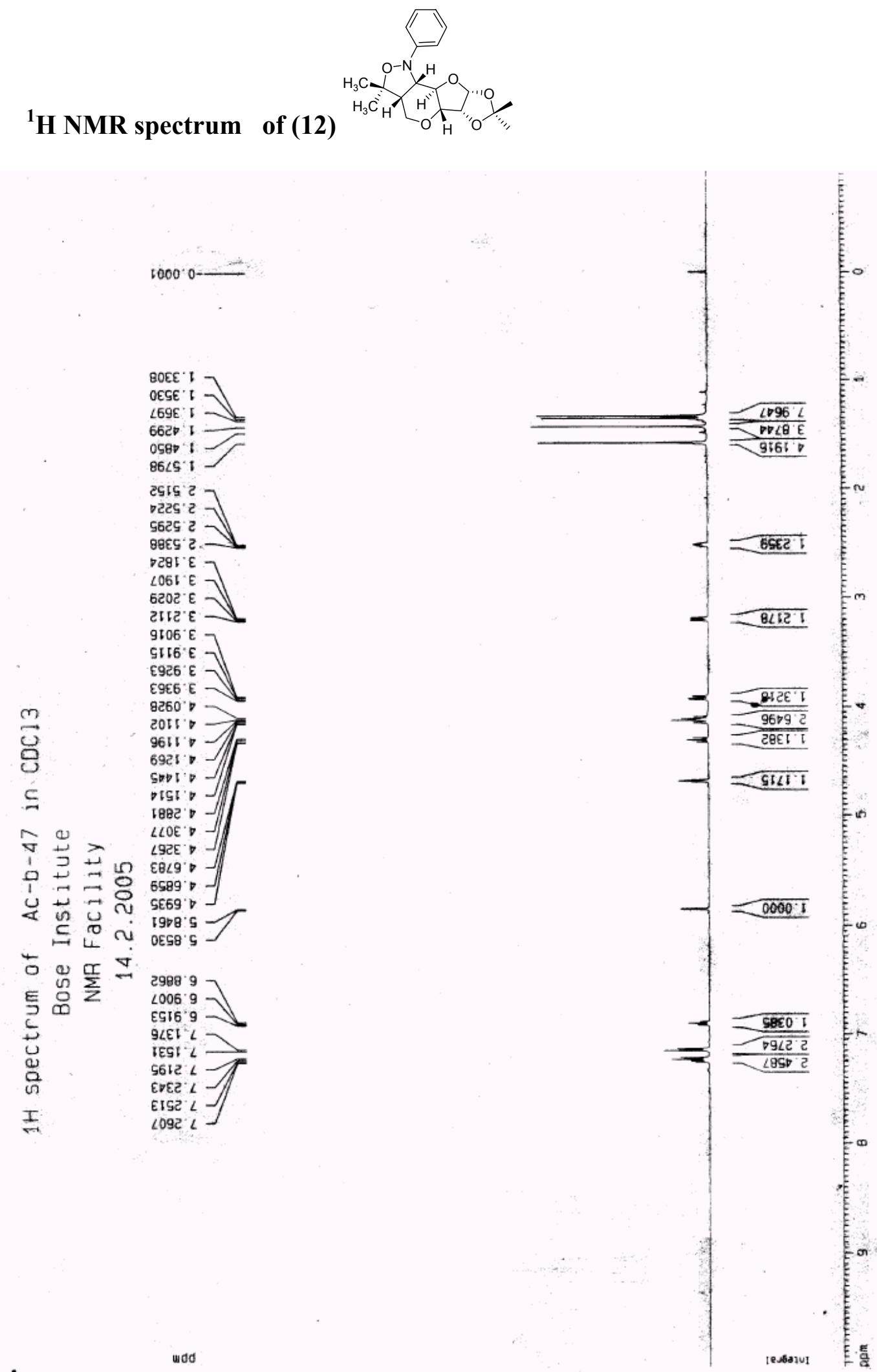
Expansion (1) of ${ }^{1} \mathrm{H}$ NMR spectrum of (12)
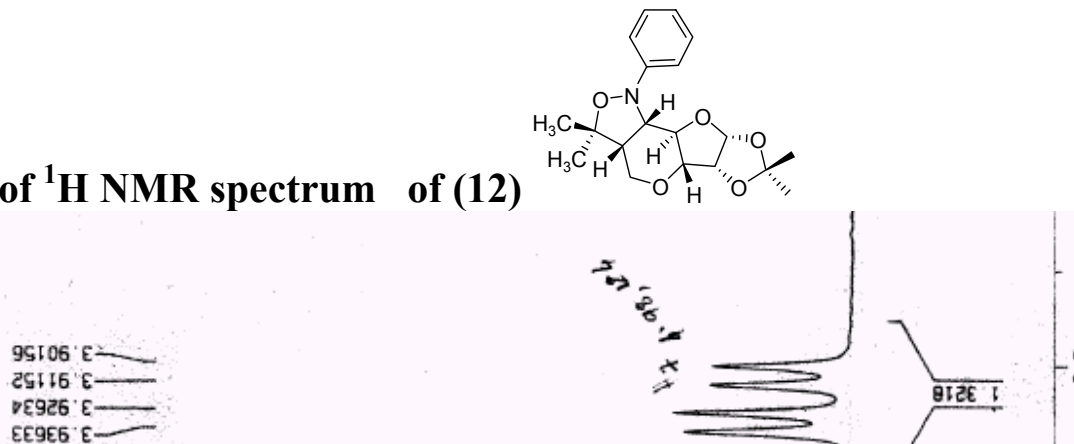

$9 \angle 690^{\circ}$,
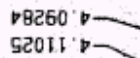

$5965.6 \div$

68927

$\angle D O O T^{\circ}$

IDISI

$\frac{m}{\mathrm{c}}$

t8ge $b$

도

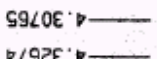

$\nabla \stackrel{4}{\nabla}>$

ए

$1 \pm=0$

琎

데

○

ज

E $\bigcirc$

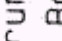

t

บั

은

$I$

EEB $\angle 9$ '

06589.

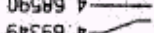

60869

SOEEL $\square$

wdd

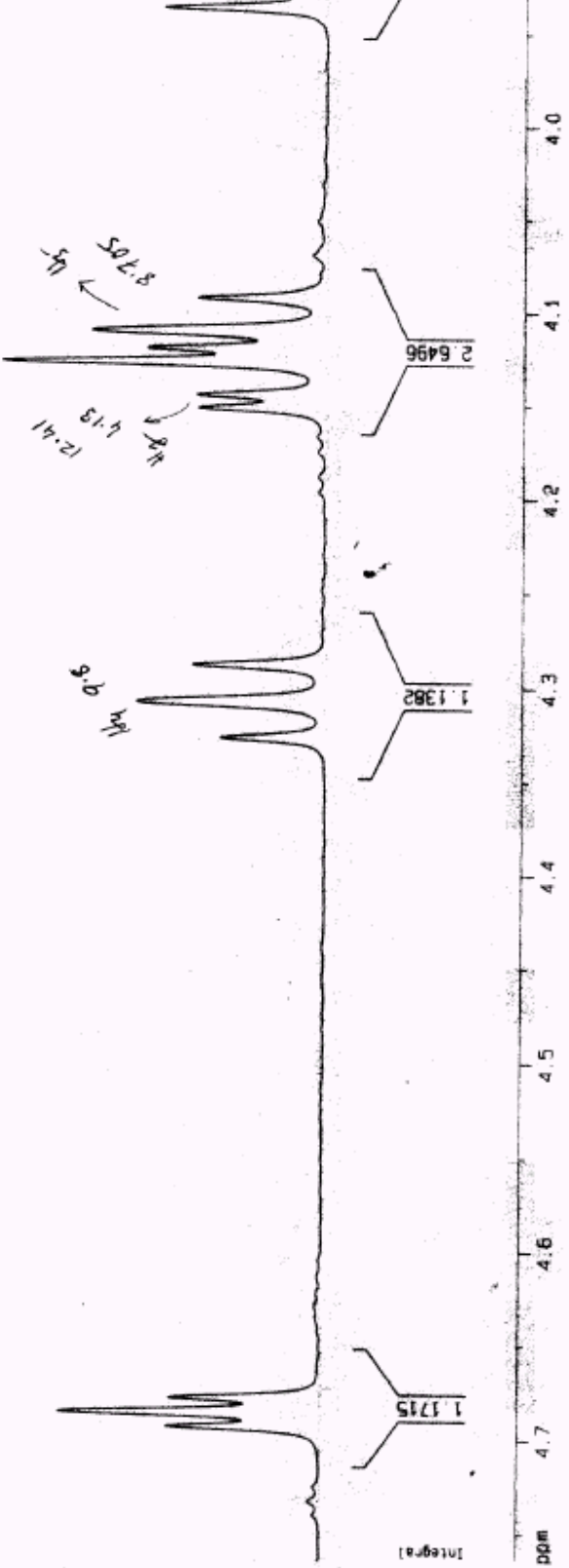



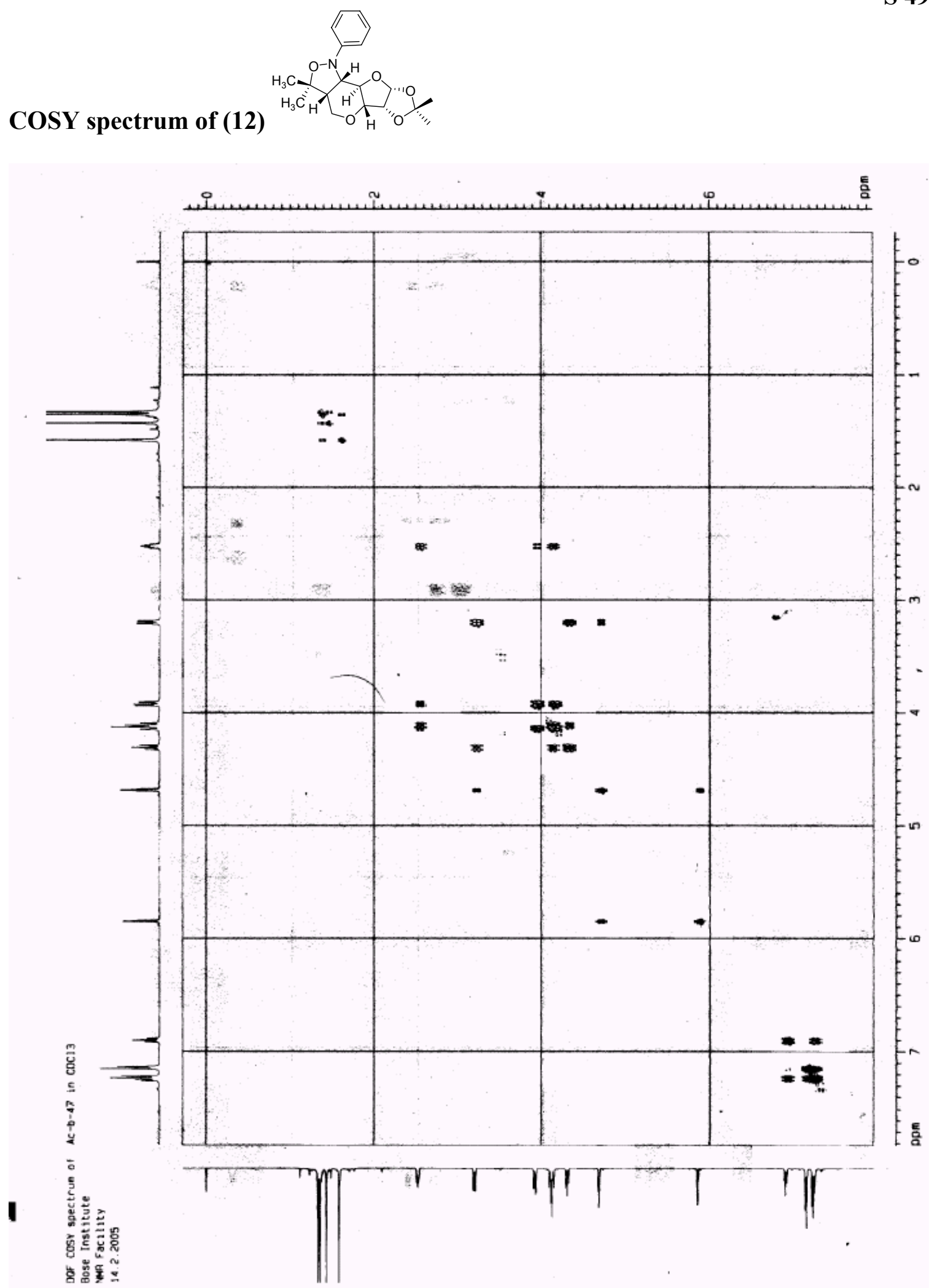
Expansion (1) of COSY spectrum of (12)

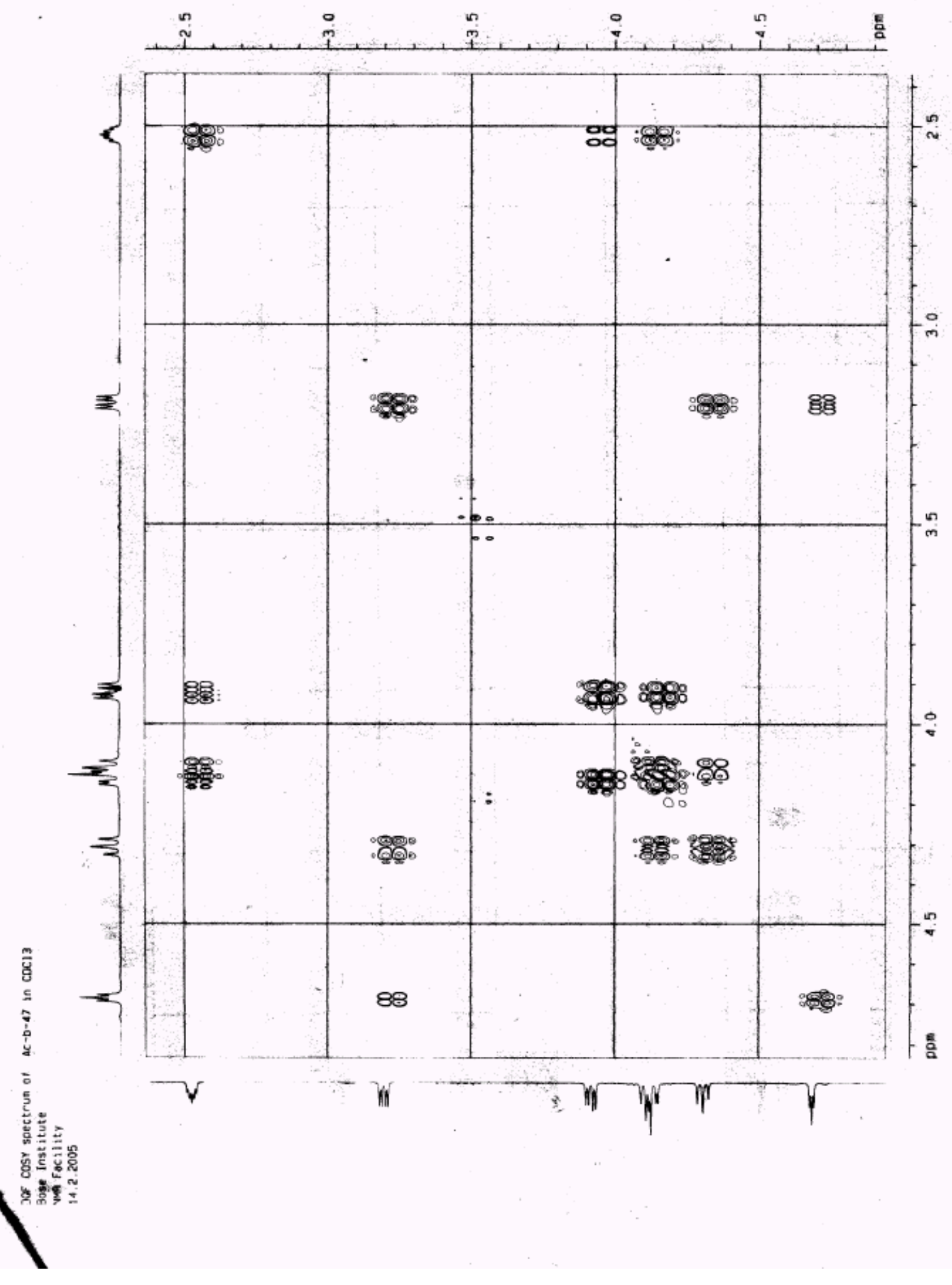




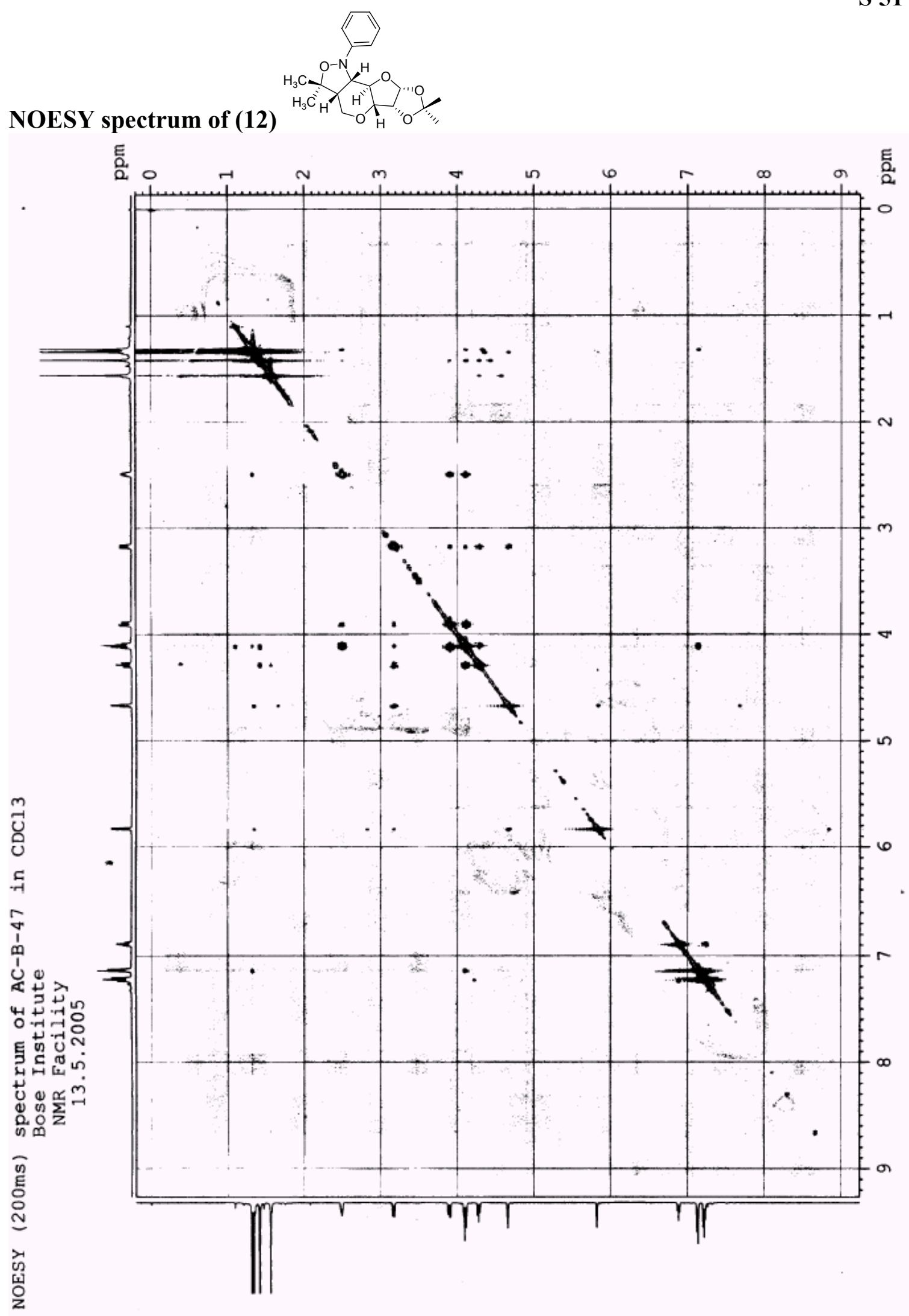


Expansion (1) of NOESY spectrum of (12)

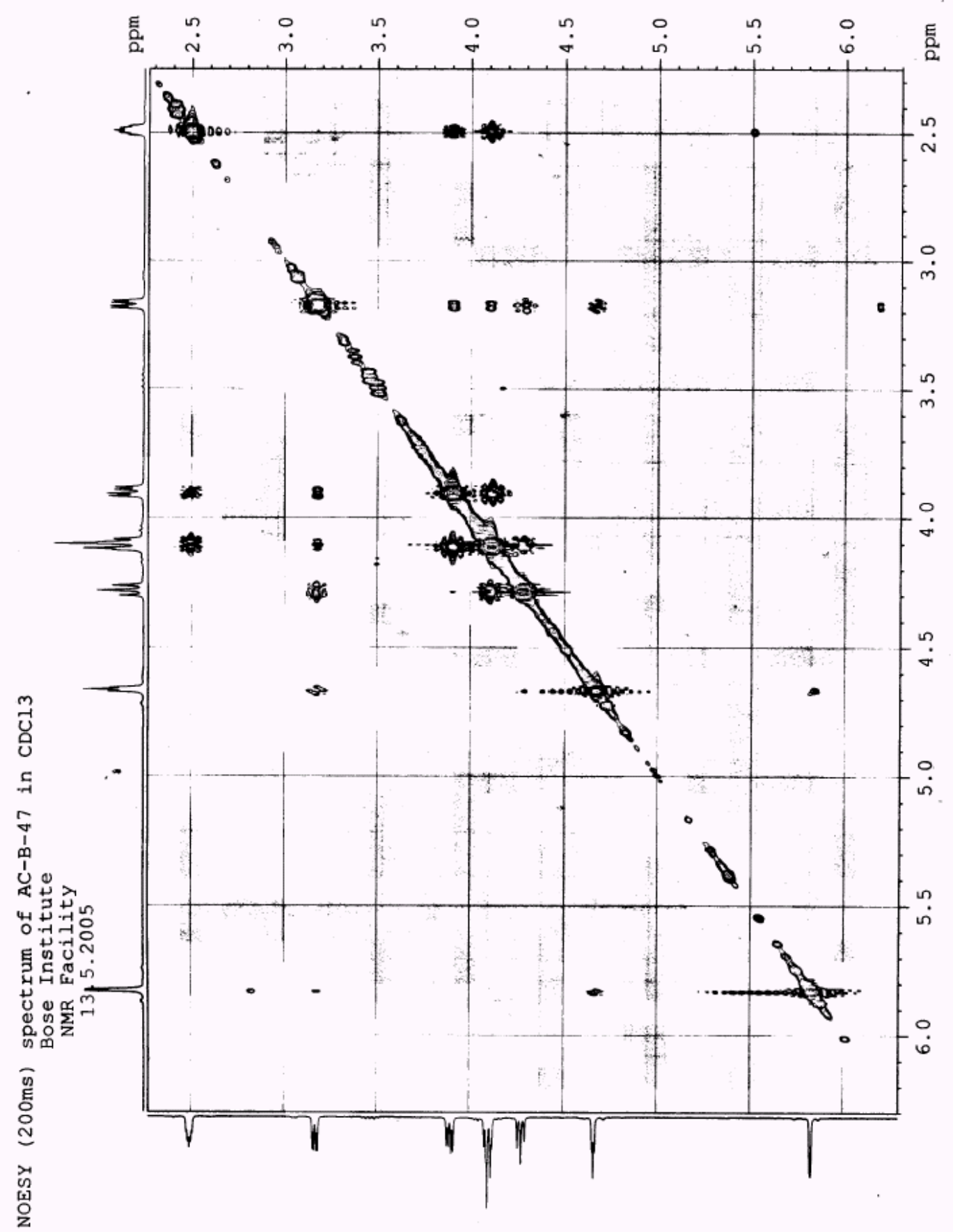


Expansion (2) of NOESY spectrum of (12)

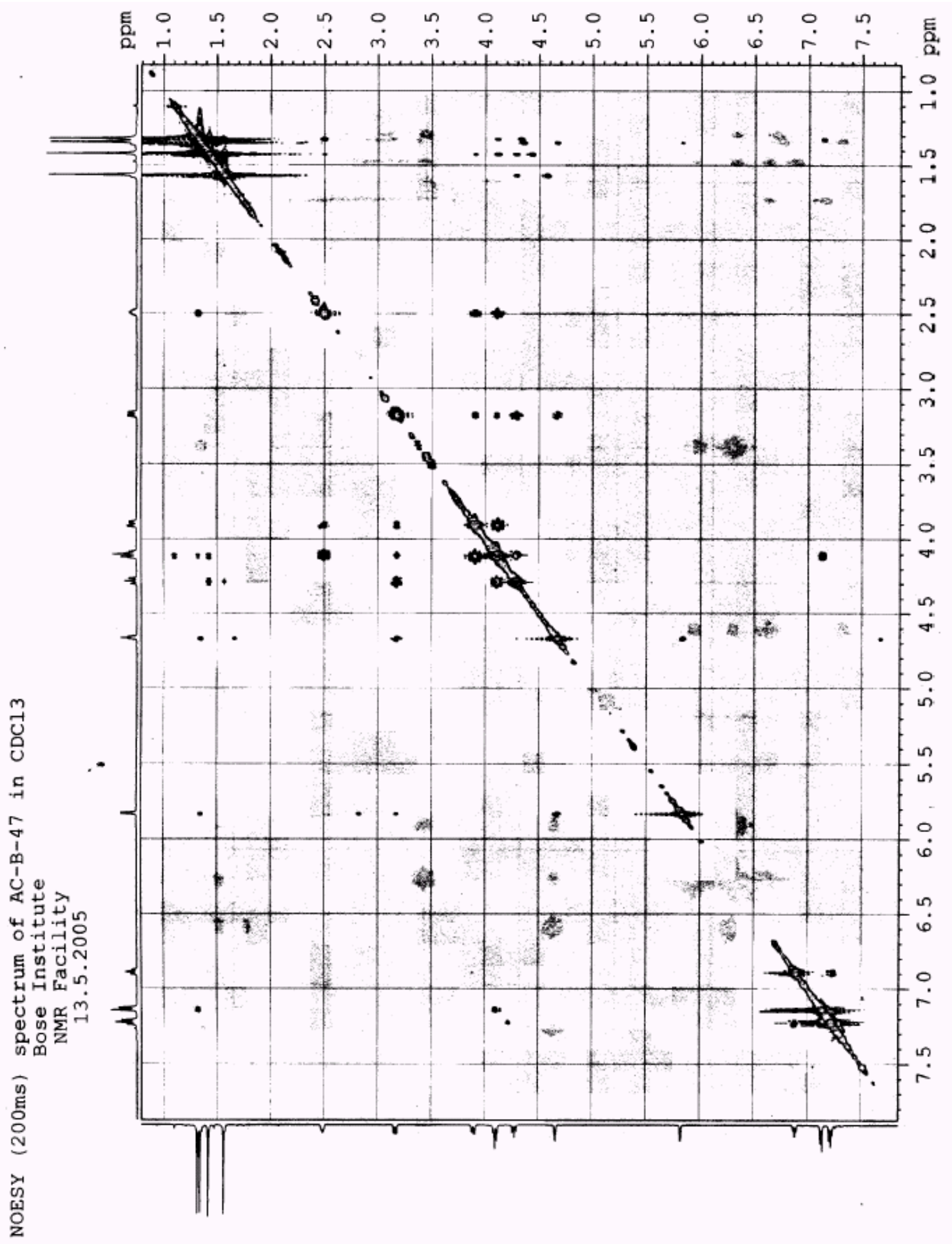


Expansion (3) of NOESY spectrum of (12)

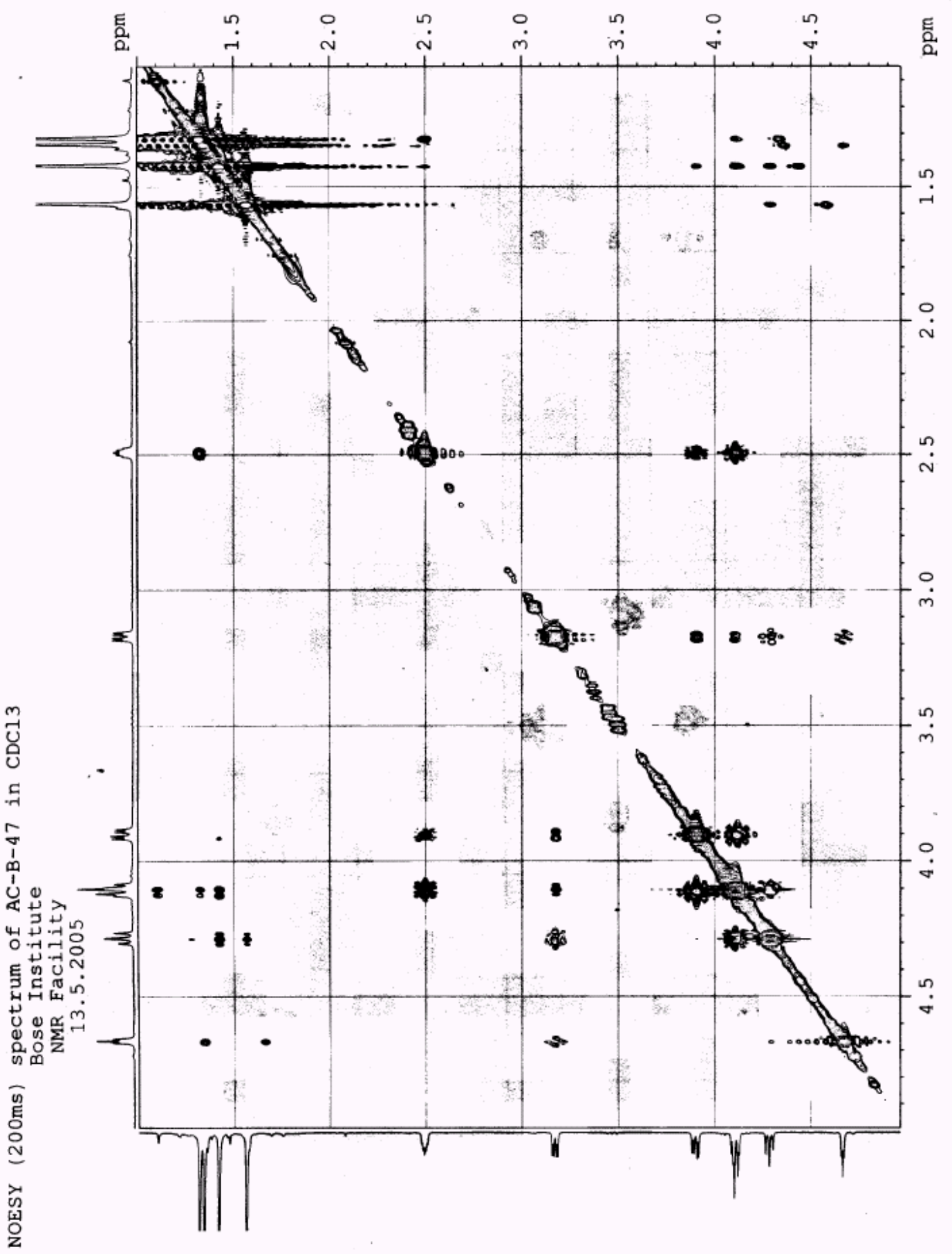


Expansion (4) of NOESY spectrum of (12)

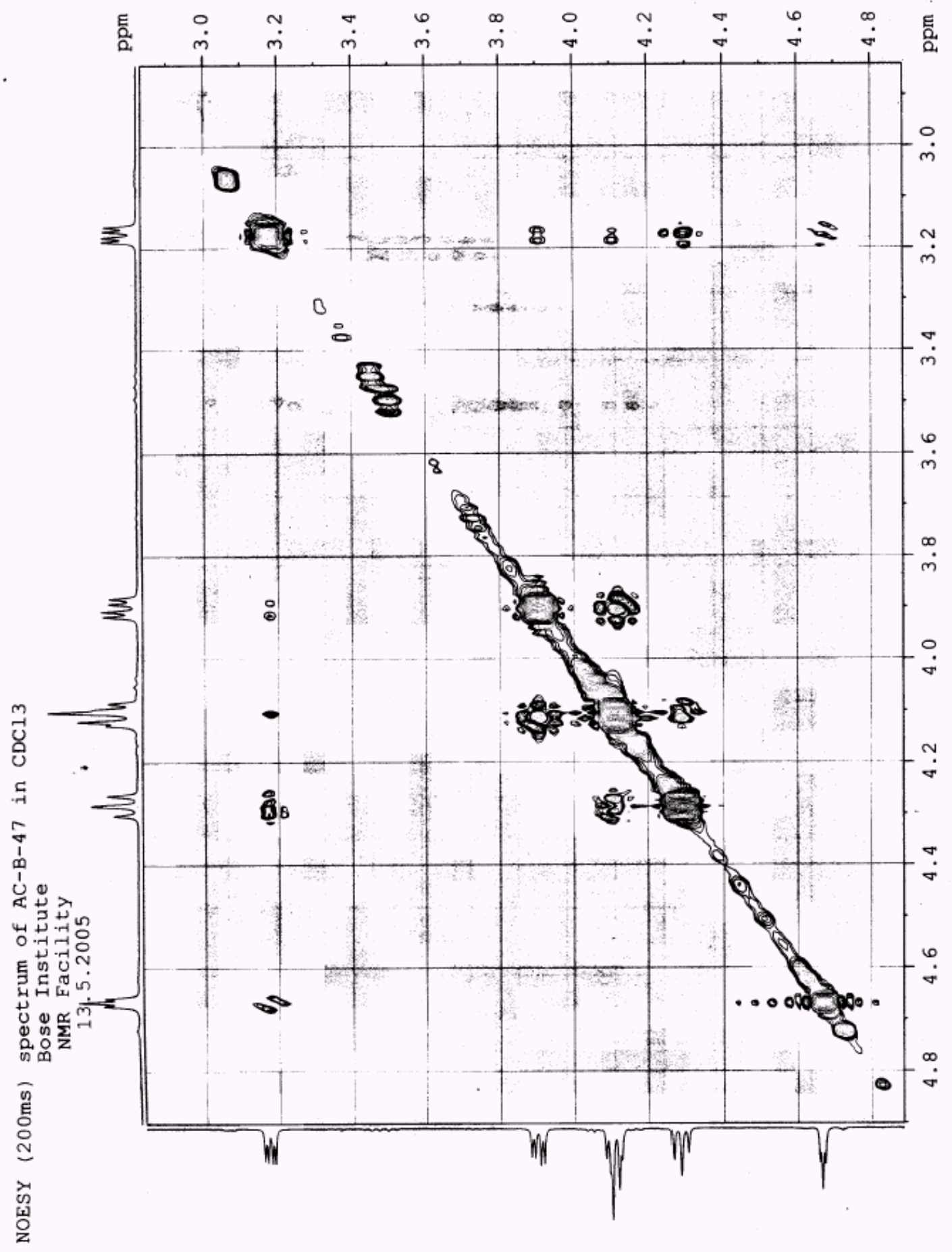




\section{${ }^{13}$ C NMR spectrum of (12)}
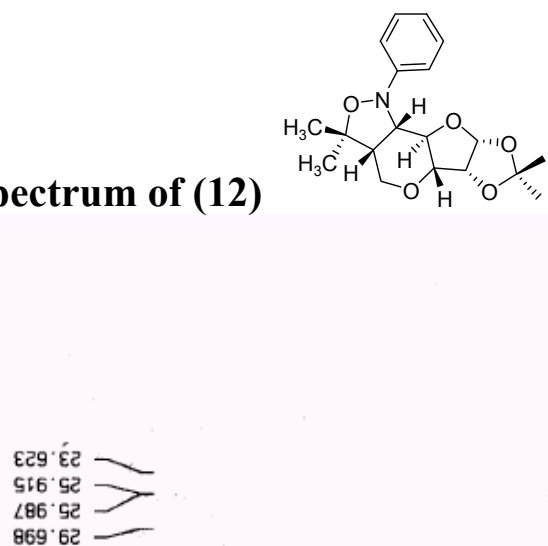

EEC 67

$9 \angle 9 \cdot 99$

969.24
180.

उ82.

IBG. 92

ํㅜㅇ

$500 . \angle L$

$\left.088^{\circ} \angle L\right]$

봉

$9066^{\circ} 28$

$\therefore \quad \nabla D E \cdot D O H$

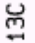

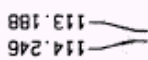

$926^{\circ} 025$

)

8ะ8 OS:-

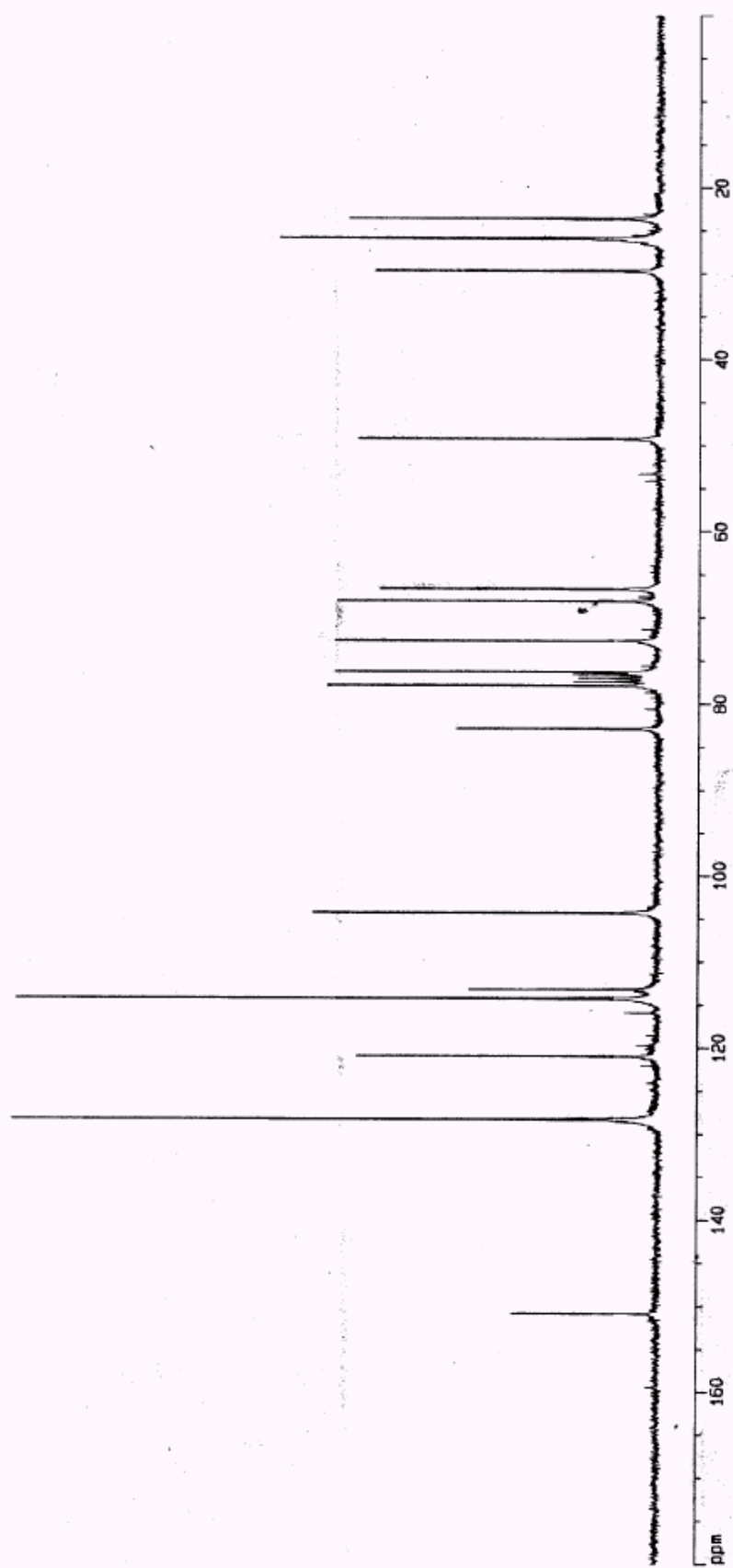

